\title{
Discrete Moving Frames on Lattice Varieties and Lattice-Based Multispaces
}

\author{
Gloria Marí Beffa ${ }^{2}$ • Elizabeth L. Mansfield ${ }^{1}$ (D
}

Received: 11 November 2015 / Revised: 11 April 2016 / Accepted: 26 September 2016 /

Published online: 31 October 2016

(C) The Author(s) 2016. This article is published with open access at Springerlink.com

\begin{abstract}
In this paper, we develop the theory of the discrete moving frame in two different ways. In the first half of the paper, we consider a discrete moving frame defined on a lattice variety and the equivalence classes of global syzygies that result from the first fundamental group of the variety. In the second half, we consider the continuum limit of discrete moving frames as a local lattice coalesces to a point. To achieve a well-defined limit of discrete frames, we construct multispace, a generalisation of the jet bundle that also generalises Olver's one-dimensional construction. Using interpolation to provide coordinates, we prove that it is a manifold containing the usual jet bundle as a submanifold. We show that continuity of a multispace moving frame ensures that the discrete moving frame converges to a continuous one as lattices coalesce. The smooth frame is, at the same time, the restriction of the multispace frame to the embedded jet bundle. We prove further that the discrete invariants and syzygies approximate their smooth counterparts. In effect, a frame on multispace allows smooth frames and their discretisations to be studied simultaneously. In our last chapter we discuss two important applications, one to the discrete variational calculus,
\end{abstract}

Communicated by Peter Olver.

This paper was supported by ELM's EPSRC Grant EP/H024018/1, GMB's NSF Grant DMS \#1405722.

$凶$ Elizabeth L. Mansfield

e.1.mansfield@kent.ac.uk

https://www.kent.ac.uk/smsas/our-people/profiles/mansfield_elizabeth.html

Gloria Marí Beffa

maribeff@math.wisc.edu

https://www.math.wisc.edu/ maribeff/

1 SMSAS, University of Kent, Canterbury CT27NF, England

2 Mathematics Department, University of Wisconsin-Madison, Madison, WI 53706, USA 
and the second to discrete integrable systems. Finally, in an appendix, we discuss a more general result concerning equicontinuous families of discretisations of moving frames, which are consistent with a smooth frame.

Keywords Discrete moving frame - Discrete invariants · Local and global syzygies of invariants - Multispace - Discrete and smooth Maurer-Cartan invariants - Finite difference calculus of variations $\cdot$ Discrete integrable systems

Mathematics Subject Classification 14H70 - 17B80 - 49M25 - 53A55 - 53C99 . 58A40

\section{Introduction}

The theory and the applications of Lie group-based moving frames are now well established, and provide an "invariant calculus" to study differential systems which are either invariant or equivariant under the action of a Lie group. Associated with the name of Cartan [7], who used repères mobile to solve equivalence problems in differential geometry, the ideas go back to earlier works, for example by Cotton [9] and Darboux [14].

Moving frames were further developed and applied in a substantial body of work, in particular to differential geometry and exterior differential systems; see for example papers by Green [19] and Griffiths [20]. From the point of view of symbolic computation, a breakthrough in the understanding of Cartan's methods for differential systems came in a series of papers by Fels and Olver [16,17], Olver [51,52], Hubert [27-29], and Hubert and Kogan [30,31], which provide a coherent, rigorous, and constructive moving frame method. The resulting differential invariant calculus is the subject of the textbook, [38]. There are now an extensive number of applications, including to the integration of Lie group invariant differential equations [38], to the Calculus of Variations and Noether's Theorem, (see for example [21,22,37]), and to integrable systems (for example [40,43-45]). Moving frame methods have been extended to Lie pseudogroups [54]. We note that the calculation of invariants of Lie group actions, using older "infinitesimal" methods, are well documented in many texts (see for example, $[2,53])$. The use of moving frames to calculate invariants compares favourably to the older methods in those cases where the frame can be explicitly calculated, since then the invariants are obtained by the substitution of the frame into the group action, while infinitesimal methods rely on the solution of first-order quasi-linear partial differential equations. Even where the frame cannot be calculated, the full symbolic "invariant calculus" using moving frames, is still available, as is explained in detail in the text, [38]. For calculating Lie symmetry groups, however, the infinitesimal methods will always be needed, as the equations for the infinitesimals are linear, while those for the group parameters themselves are highly nonlinear.

The first results for the computation of discrete invariants using group-based moving frames were given by Olver who called them joint invariants in [52]; modern applications to date include computer vision [50] and numerical schemes for systems with a Lie symmetry $[34-36,41,56]$. While moving frames for discrete applications 
as formulated by Olver do give generating sets of discrete invariants, the recursion formulae for differential invariants which were so successful for the application of moving frames to calculus-based results, do not generalise well to joint invariants. In particular, joint invariants do not seem to have recursion formulae under the shift operator that are computationally useful. To overcome this problem, the authors, together with Jing Ping Wang, introduced the notion of a discrete moving frame which is essentially a sequence of frames [42]. In that paper we prove discrete recursion formulae for small computable generating sets of invariants, which we call the discrete Maurer-Cartan invariants, and investigated their syzygies, that is, their recursion relations. The main application to date has been to discrete integrable systems, with the authors of [47] proving that discrete Hamiltonian structures for $W_{n}$-algebras can be obtained via a reduction process. We note that a sequence of moving frames was also used in [35] to minimise the accumulation of errors in an invariant numerical method.

In this paper, we extend the theory of discrete moving frames in two ways. The first is to consider a discrete moving frame defined on a lattice variety, which can be thought of as the vertices, or 0-cells, together with their adjacency information, in a discrete approximation of a manifold. We describe their associated cross sections and define Maurer-Cartan invariants and local syzygies. In Sect. 3.2 we further classify global syzygies and prove that they are associated with topological aspects of the variety, like representatives of the discrete fundamental group of the lattice variety, with properties like twisting.

The second extension, beginning in Sect. 4 and for the rest of the paper, is to consider families of discrete frames and how their continuum limits may define smooth frames. Our interest in this second case is how discrete invariants and their recursion relations limit to differential invariants and their differential syzygies. We show not only that the limits exist, but also that a well-defined continuum limit of discrete frames may be achieved by embedding it in a smooth family of discrete ones.

In order to provide a general framework, we construct a manifold which we call the lattice-based multispace and which generalises, in some sense, the curve-based multispace of Olver [51]. The multispace is a generalisation of the jet bundle which contains the jet bundle as a submanifold. It also contains the space of lattices as an open subset. The main problem with the definition of the lattice multispace is the fact that multivariate interpolation is not well-defined in general. To avoid this problem we restrict the lattices to sets of points covered by the general construction of de Boor and Ron [11-13], to what we call corner lattices. A corner lattice is one with just enough data to guarantee the approximation of a smooth jet. We restrict as well the types of coalescencing that can take place to be those along hyperplanes. We show that de Boor and Ron's interpolating family is well defined on corner lattices and is smooth under coalescing, smooth in the sense that the associated Lagrange polynomials converge to the Hermite ones as the vertices of the lattice coalesce (Fig. 1). Once the choice of lattice and conditions on coalescing are settled, we can use the interpolating coefficients to define the coordinate system in the multispace manifold. We notice that one can possibly consider other forms of lattices and coalescing, and that our theory will hold true as far as the smoothness of de Boor-Ron's family is preserved. 
(a)

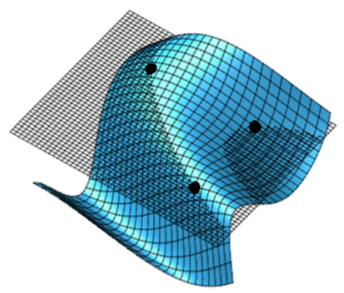

(b)

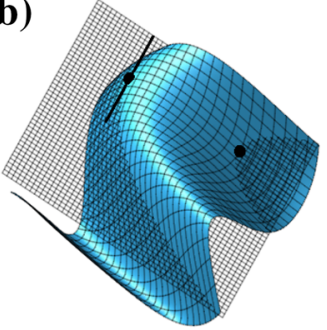

(c)

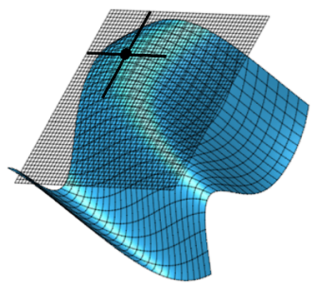

Fig. 1 Under coalescence of the points at which the interpolation is calculated, Lagrange interpolation becomes Hermite interpolation, ending with the Taylor approximation to a surface when all the interpolation points coalesce. By taking coordinates for the lattice-based multispace to be the grid points and the Lagrange interpolation coefficients, the jet bundle is naturally embedded. a Lagrange interpolation, b Hermite interpolation, c Taylor approximation

Once the multispace is proved to be a smooth manifold, we can naturally define a group action on it, and hence we can talk about smooth moving frames on multispaces. A moving frame on the lattice-based multispace is, simultaneously, a smooth moving frame defined on the jet bundle, and a frame defined on local difference approximations to the derivatives, depending on what point of the multispace the moving frame is evaluated. By defining a moving frame on multispaces, one has simultaneously the full power of both the smooth and the discrete frames, and the smoothness of the multispace frame will ensure that we can move freely between discrete, discrete/differential and smooth frames, ensuring that the discrete frame converges to the continuous one as the points in the corner lattices coalesce to create the jet. We also show that the continuity carries over to invariants and syzygies as well. Therefore, any smooth geometric construction carried out with a multispace lattice, invariants and syzygies, ensures that the final discrete, or discrete/differential result, is an approximation of the corresponding continuous construction.

In Sect. 5 we use the multispace construction in two different applications. The first application is to a class of finite difference variational shallow water systems, which have both the correct continuum limit as well as the necessary symmetries for Noether's theorem to yield conservation laws for energy, and linear and angular momenta, in both the finite difference case and the smooth limit. This is motivated by the desire to achieve an analogue of the conservation of potential vorticity in a numerical approximation to these equations.

The second application concerns discretisations of completely integrable systems. Most well-known completely integrable PDEs are linked to some geometric background and the PDE can be interpreted as, for example, the equation induced on invariants by a geometric evolution of curves, or like the Codazzi-Mainardi equations, are associated with the geometry of some type of surface. Discrete lattice systems also have similar interpretations [3]. The question to ponder is whether or not the same geometric construction performed in the continuous case to generate the PDEs can be carried out in the discrete case, while guaranteeing that the result will be a discretisation of the PDE; this might be useful as a base to study the more interesting questions of when the discretisation will be also completely integrable. Here we show two such processes. The Boussinesq equation is induced on centro-affine invariants 
by an evolution of star-shaped curves. We construct the multispace version of the construction to obtain a geometric discretisation. We show that a modification of the construction generates an integrable discretisation which appeared in [47]. The study of how these different discretisations might be related is underway. In the second example we describe the multispace version of the well-known construction of the Sine-Gordon equation as the Codazzi-Mainardi equations for Euclidean surfaces of negative constant curvature. This interpretation has been widely used to study pseudospherical surfaces as generated by solutions of Sine-Gordon, see [8] and [57]. Every step of the construction is guaranteed to discretise the continuous version, while preserving the geometric meaning of the elements involved. The Sine-Gordon is in fact one of several equations describing the surface, but which decouples from the others. In this discretisation the equations remains coupled and its integrability is not clear, but the construction itself is a non-trivial example of the use of mixed discretesmooth moving frames. The connection between multispace and integrability is under study.

Finally, in an appendix, we discuss a more general result concerning the discretisation of smooth moving frames, and the continuum limit of equicontinuous families of discrete moving frames, with an example.

\section{Background}

\subsection{Moving Frames}

Given a Lie group $G$ acting on a manifold $M$ with a left action, so that

$$
G \times M \rightarrow M, \quad h \cdot(g \cdot z)=(h g) \cdot z,
$$

one can define a right (resp. left) group-based moving frame as a map which is equivariant with respect to the action on $M$ and the inverse right (resp. left) action of $G$ on itself, specifically,

$$
\rho: M \rightarrow G, \quad \rho(g \cdot z)=\rho(z) g^{-1} \quad(\operatorname{resp} . \rho(g \cdot z)=g \rho(z)) .
$$

We call such an equivariant map a right (resp. a left) moving frame. The inverse of a right moving frame is a left one, and vice versa.

Given a group $G$ acting on a manifold $M$, the existence of a moving frame on the open subset $U \subset M$ is guaranteed if:

(i) the orbits of the group action all have the same dimension and foliate $U$,

(ii) there is a transverse cross section $\mathcal{K}$ to the orbits such that for each orbit $\mathcal{O}$, the intersection $\mathcal{O} \cap \mathcal{K}$ contains a single point, and

(iii) the group element taking $z \in \mathcal{O}(z)$ (where $\mathcal{O}(z)$ is the orbit through $z$ ) to $\mathcal{O}(z) \cap$ $\mathcal{K}$, is unique.

In this case, a right moving frame $\rho: U \rightarrow G$ is given by $\rho(z) \cdot z \in \mathcal{K}$, that is, $\rho(z)$ is the unique element of $G$ taking $z$ to the unique element of $\mathcal{K} \cap \mathcal{O}(z)$. Since $\mathcal{K}$ is transverse to the orbits, the frame defines local coordinates given by $z \mapsto(\rho(z), \rho(z) \cdot z) \in G \times \mathcal{K}$. 
In the continuous case of moving frames, the manifold $M$ could be the jet space $J^{(\ell)}\left(\mathbb{R}^{p}, M\right)$. In this case it is known [17] that provided the action is locally effective on subsets, as $\ell$ grows the prolonged action of $G$ on $J^{(\ell)}\left(\mathbb{R}^{p}, M\right)$ becomes locally free. The work of Boutin [5] discusses what happens for products $M^{q}$ as $q$ grows, with $G$ acting with the diagonal action. In any event, we make the assumption that for large enough dimension, there is a neighbourhood of the identity in the group in which a moving frame can be obtained locally.

A common way to obtain the moving frame is through a normalisation process. One can describe normalisation equations as those defining the transverse section, $\mathcal{K}$, to the orbits of the group. If the normalisation equations are given as $\{\Phi=0\}$, then the conditions above for the existence of a moving frame are the conditions under which the implicit function theorem can be applied to solve $\Phi(g \cdot z)=0$ for $g=\rho(z)$. Since both $g=\rho(h \cdot z)$ and $g=\rho(z) h^{-1}$ solve $\Phi(g \cdot(h \cdot z))=0$, and the implicit function guarantees a unique solution, then $\rho(h \cdot z)=\rho(z) h^{-1}$, that is, $\rho$ is equivariant. Typically, the normalisation equations, for which $\mathcal{K}$ is the zero set, are algebraic. Indeed, in many applications, the cross section is a coordinate plane, so that the normalisation equations involve certain coordinates being set to a constant. Since there will be many transverse cross sections to the orbits, the choice of $\mathcal{K}$ can greatly decrease (or increase) the calculations involved. Part of the "art" of the moving frame in applications is the choice of cross section, or equivalently, the choice of normalisation equations.

Given a moving frame (left or right) one can generate all possible invariants of the action. Indeed, if $\rho$ is a right moving frame, the expressions

$$
\rho(u) \cdot v
$$

for any $u, v \in M$ are clearly invariant; their coordinates are called the normalised invariants. One can easily see that any invariant of the action is a function of these, using the replacement rule: If $I: M \rightarrow \mathbb{R}$ is invariant under the action, so that $I(g \cdot v)=I(v)$ for all $g \in G$, then setting $g=\rho(u)$, one obtains

$$
I(\rho(u) \cdot v)=I(v) .
$$

Different choices of the manifold $M$ gives rise to different familiar cases. For example, if $M$ is the jet space $J^{(\infty)}\left(\mathbb{R}^{p}, P\right)$ for some manifold $P$ where $G$ acts, and $G$ acts on $M$ via the natural prolonged action given by the chain rule, then $\rho$ would generate moving frames on $p$-submanifolds and the invariants will be standard differential invariants (curvatures, torsions, etc). If $M=P^{k}$ is the Cartesian product of a manifold $P$ where $G$ acts, and $G$ acts on $M$ through the diagonal action, then the invariants are the so-called joint invariants (see [52]).

Remark 2.1 In this portion of the paper, we are interested in the induced action on $N$-gons, that is, on sets of $N$ points in $M$, or alternatively, an element of $M^{N}$.

The authors of [42] defined discrete moving frames, essentially a choice of group element associated with each vertex in an equivariant way. The discrete moving frame

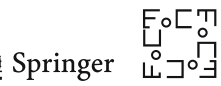


can be defined to act naturally under the shift operator, greatly simplifying calculations with discrete (difference) equations. We next review this definition.

Let $G^{N}$ denote the Cartesian product of $N$ copies of the group $G$. Allow $G$ to act on the left on $G^{N}$ using the diagonal action $g \cdot\left(g_{r}\right)=\left(g g_{r}\right)$. We also consider what we have called the "right inverse action" $g \cdot\left(g_{r}\right)=\left(g_{r} g^{-1}\right)$.

Definition 2.2 (Discrete moving frame) We say a map

$$
\rho: M^{N} \rightarrow G^{N}
$$

is a right (resp. left) discrete moving frame if $\rho$ is equivariant with respect to the diagonal action of $G$ on $M^{N}$ and the inverse right (resp. left) diagonal action of $G$ on $G^{N}$. Since $\rho\left(\left(x_{r}\right)\right) \in G^{N}$, we will denote by $\rho_{s}$ its $s$ th component, that is $\rho=\left(\rho_{s}\right)$, where $\rho_{s}\left(\left(x_{r}\right)\right) \in G$ for all $s$. Equivariance means,

$$
\rho_{S}\left(g \cdot\left(x_{r}\right)\right)=\rho_{s}\left(\left(g \cdot x_{r}\right)\right)=\rho_{S}\left(\left(x_{r}\right)\right) g^{-1} \quad\left(\text { resp. } g \rho_{s}\left(\left(x_{r}\right)\right)\right)
$$

for every $s$. Clearly, if $\rho=\left(\rho_{s}\right)$ is a right moving frame, then $\varrho=\left(\rho_{s}^{-1}\right)$ is a left moving frame.

Remark 2.3 In any given application, it is advisable to ensure the parity of an action and of the equivariance of a frame; see [38] for a discussion of the subtleties involved. In what follows, we will use $\rho$ to denote a right frame, and $\varrho$ to denote a left frame.

As in the original group-based moving frame definition, if $\left(u_{S}\right) \in M^{N}$, one can define invariants,

$$
I_{s}^{r}=\rho_{s} \cdot u_{r}
$$

for a right frame, or $I_{s}^{r}=\varrho_{s}^{-1} \cdot u_{r}$ for a left frame. The coordinates of these invariants for any $r$ generate all other invariants even when s is fixed (see [42]). We note that the action induces an action on the coordinate functions, the same as it induces an action on any function, specifically, $g \cdot f\left(u_{r}\right)=f\left(g \cdot u_{r}\right)$. The components of $I_{s}^{r}$ will be invariant as $I_{s}^{r}$ is, and they are called the normalised invariants.

We next describe a smaller set of invariants, the so-called Maurer-Cartan invariants.

Definition 2.4 Let $\left(\rho_{s}\right)$ be a right (resp. left) discrete moving frame evaluated along an $N$-gon. Then the element of the group

$$
K_{s}=\rho_{s+1} \rho_{s}^{-1} \quad\left(\text { resp. } \varrho_{s}^{-1} \varrho_{s+1}\right)
$$

is called the right (resp. left) $s$-Maurer-Cartan element for $\rho$ (resp. $\varrho$ ). We call the equation $\rho_{s+1}=K_{s} \rho_{s}\left(\varrho_{s+1}=\varrho_{s} K_{s}\right)$ the discrete right (resp. left) $s$-Frenet-Serret equation. 
(a)

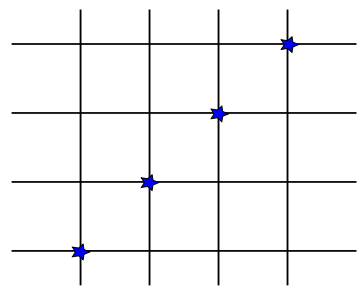

(c)

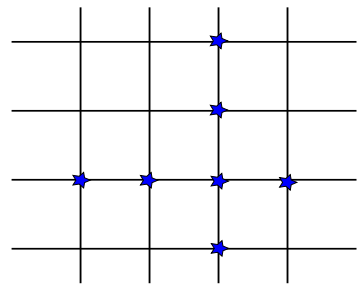

(b)

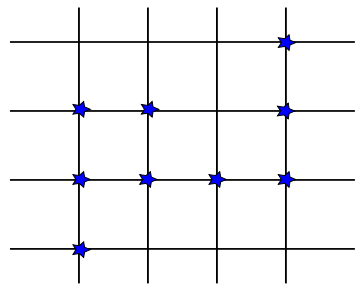

(d)

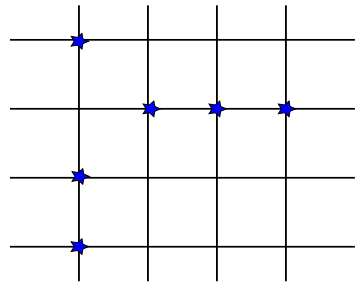

Fig. 2 In $\mathbf{b}$ and $\mathbf{c}, \Gamma$ is connected. The subsets in $\mathbf{a}, \mathbf{d}$ are not connected; the subset in $\mathbf{a}$ has four components and that in $\mathbf{d}$ has three

The coordinates of the Maurer-Cartan elements, together with the normalised invariants $I_{s}^{S}$, generate all other invariants. See [42] for more details. Note that for $G \subset G L(n, \mathbb{R})$ a matrix group, the Maurer-Cartan invariants will be the components of the Maurer-Cartan matrices.

\subsection{Lattices}

Lattices are subsets of $\mathbb{Z}^{p}$ with a variety of properties. We first define adjacency.

Definition 2.5 Two points $\mathbf{m}, \mathbf{n} \in \mathbb{Z}^{p}$ are said to be adjacent if

$$
\sum_{j}\left|m_{j}-n_{j}\right|=1
$$

Definition 2.6 We say that a subset $\Gamma \subset \mathbb{Z}^{p}$ is a connected lattice if it consists of a single point, or, if between any two points $\mathbf{m}_{1}, \mathbf{m}_{2} \in \Gamma$ there is a path, $\mathbf{m}=$ $\mathbf{n}_{1}, \mathbf{n}_{2}, \ldots \mathbf{n}_{N}=\mathbf{m}_{2}$ such that $\mathbf{n}_{i}$ is adjacent to $\mathbf{n}_{i+1}$ for $i=1, \ldots, N-1$.

This definition is illustrated in Fig. 2. Natural operators on $\mathbb{Z}^{p}$ are the well-known shift operators, namely $\mathcal{T}_{i}, i=1, \ldots, p$ where

$$
\mathcal{T}_{i}\left(n_{1}, \ldots, n_{i}, \ldots, n_{p}\right)=\left(n_{1}, \ldots, n_{i}+1, \ldots, n_{p}\right)
$$

We will also consider lattices in a manifold.

Definition 2.7 The image of a lattice $\Gamma \subset \mathbb{Z}^{p}$ in a manifold $M$ by a map $\Phi: \Gamma \rightarrow M$ is denoted by $L$, and is also called a lattice. We assume this map to be injective, a 
condition which will be relaxed under controlled conditions in the second part of this paper. The adjacency in $L$ is determined from that of $\Gamma$.

Since we will be working in coordinate charts, we can assume from the beginning that the lattice $L \subset M$ is contained within one coordinate chart for the manifold, so that for all practical purposes we can assume the manifold is $\mathbb{R}^{n}$, or a parametrised surface with the parameters serving as local coordinates. We will also assume that lattices are connected as one can study each connected component separately.

The lattice itself does not need to be covered with one lattice neighbourhood,however. We define next a lattice variety, which will allow us to work on lattice models of spheres and tori.

Remark 2.8 Another name for our lattice variety could be 'lattifold', since we define it to be a manifold like object but modelled on $\mathbb{Z}^{p}$ rather than $\mathbb{R}^{p}$. The construction given here is related to that given in [39].

Definition 2.9 A lattice variety $\mathcal{L} \subset M$ is a set that can be covered by a countable number of lattices $\mathcal{L}_{\alpha} \subset M$, each of which is the image under an injection $\phi_{\alpha}$ of a connected lattice $\Gamma_{\alpha} \subset \mathbb{Z}^{p}$ for some fixed $p$. Every adjacency in $\mathcal{L}$ is contained in at least one of the $\mathcal{L}_{\alpha}$. Furthermore, in the overlap $\mathcal{L}_{\alpha} \cap \mathcal{L}_{\beta}$, the gluing map $\phi_{\alpha} \circ \phi_{\beta}^{-1}$ preserves adjacency. We call $\left(\mathcal{L}_{\alpha}, \phi_{\alpha}\right)$ a local lattice coordinate system.

Lattice coordinates essentially introduce a local order in the lattice (inherited from $\mathbb{Z}^{p}$ through $\phi_{\alpha}$ ) so one can clearly define shifts. We say that a shift map is defined at a point in $\mathcal{L}$, if it is defined in at least one chart. Since the chart interchange maps preserve adjacency, the existence of a shift map is well defined.

\section{Moving Frames on Lattices and Lattice Varieties}

Let $\mathcal{L}$ be a lattice variety and let $N$ be the number of vertices in $\mathcal{L}$, which we assume to be either finite or at most countable. Let $\mathcal{L}_{N}$ be the set of $p$-lattice varieties in $M$ with $N$ vertices.

\subsection{Moving Frames, Invariants and Maurer-Cartan Invariants}

Let $G$ be a group acting on $\mathcal{L}_{N}$ (for example, if the lattice lives inside a manifold with a group action, the action would be the one induced on the lattice), and for simplicity assume that it is a left action (that is, $g \cdot(h \cdot u)=(g h) \cdot u$. A parallel description can be made for right actions.

A discrete moving frame will associate an element of the group to each vertex in the lattice in an equivariant fashion.

Definition 3.1 (Moving frames on lattices) Let $\mathcal{U}$ be a subset of $\mathcal{L}_{N}$. We say

$$
\rho: \mathcal{U} \rightarrow G^{N}
$$


is a right (resp. left) discrete moving frame on $\mathcal{U}$ whenever $\rho$ is equivariant with respect to the action of $G$ on $\mathcal{L}_{N}$ and the inverse right (resp. left ) diagonal action of $G$ on $G^{N}$. That is, if $\rho=\left(\rho_{i}\right)_{i=1}^{N}$ denotes the components of $\rho$ in $G^{N}$, then

$$
\rho_{i}(g \cdot \mathcal{L})=\rho_{i}(\mathcal{L}) g^{-1} \quad\left(\operatorname{resp} . \rho_{i}(g \cdot \mathcal{L})=g \rho_{i}(\mathcal{L})\right)
$$

with $|\Gamma|=N$.

If $\rho$ is a right frame, then $\varrho=\rho^{-1}$ is a left frame, and it suffices to develop the theory for only one of the parities. Henceforth, we restrict ourselves to right frames. In general, moving frames exist only locally, which is the reason why we need to restrict its domain in $\mathcal{L}_{N}$. Given a lattice variety $\mathcal{L}$, and a coordinate system indexed by $\Gamma \subset \mathbb{Z}^{p}$, the moving frame $\rho$ assigns group elements at each vertex (thus $N$ of them). We will call $\rho_{R}$ the moving frame at the vertex $R \in \Gamma$. Note when the index is applied we are assuming the use of local variety coordinates.

It is a simple matter to go from a moving frame to a discrete moving frame by taking a family of cross sections, one per lattice vertex, as stated in the following result.

Proposition 3.2 Let $\left\{\mathcal{S}_{R} \subset M^{N} \mid R \in \Gamma\right\}$, be a family of sections, indexed locally by $\Gamma$, with $\mathcal{S}_{R}$ transverse to the orbit of $G$ at $\mathcal{L}$ viewed as a point of $M^{N}$ (recall that $G$ acts on $M^{N}$ by the diagonal action; transversality is with respect to the orbit in $M^{N}$ ). Let $g=\left(g_{R}\right) \in G^{N}$ be uniquely determined by the condition

$$
g_{R} \cdot \mathcal{L} \in \mathcal{S}_{R}
$$

for $\mathcal{L} \in \mathcal{L}_{N}$ and $R \in \Gamma$ in some coordinate system for $\mathcal{L}$. Then $\left(g_{R}\right)=\left(\rho_{R}\right)$ is a local right discrete moving frame.

The proof of this statement is straightforward from the discussion of the moving frame. We note that a moving frame is defined as an element of the group on the entire lattice, but normalisation equations give frames defined only locally. The interchange maps from one domain to another will play a role in what follows.

Remark on Notation From now on a multi-index will denote the use of local lattice coordinates, while the lack of it will indicate global definitions. Also, we will denote by $\rho_{R}$ an individual component of $\rho$, or the moving frame at the vertex $u_{R}$. Notice also that even though we will denote by $\rho(\mathcal{L})$ the moving frame along $\mathcal{L}$, each $\rho_{R}$ will, in the examples, depend on only finitely many vertices.

Example 3.3 Consider 1-lattices-or polygons - in the Euclidean plane. The group $E(2)$ can be identified with the subgroup of $\operatorname{GL}(3, \mathbb{R})$ given by

$$
g=\left(\begin{array}{ll}
1 & 0 \\
b & \Theta
\end{array}\right)
$$

with $\Theta \in O(2)$ and $b \in \mathbb{R}^{2}$. It acts on $\mathbb{R}^{2}$ as

$$
\left(\begin{array}{ll}
1 & 0 \\
b & \Theta
\end{array}\right)\left(\begin{array}{l}
1 \\
u
\end{array}\right)=\left(\begin{array}{c}
1 \\
\Theta u+b
\end{array}\right)
$$

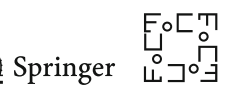


with $u \in \mathbb{R}^{2}$. We choose as our transverse cross section, the one given in coordinates by $\rho_{n} \cdot u_{n}=0$ and $\rho_{n} \cdot u_{n+1}=e_{1}\left\|\Delta u_{n}\right\|$, where $\Delta u_{n}=u_{n+1}-u_{n}$. If $\rho_{n}$ is displayed in the matrix representation as in (2), solving the system

$$
\rho_{n} \cdot u_{n}=\Theta_{n} u_{n}+b_{n}=0, \quad \rho_{n} \cdot u_{n+1}=\Theta_{n} u_{n+1}+b_{n}=\Theta_{n} \Delta u_{n}=\left\|\Delta u_{n}\right\| e_{1}
$$

results in the left moving frame

$$
\varrho_{n}=\rho_{n}^{-1}=\left(\begin{array}{cc}
1 & 0 \\
-\Theta_{n}^{-1} b_{n} & \Theta_{n}^{-1}
\end{array}\right)=\left(\begin{array}{ccc}
1 & 0 & 0 \\
\left\|\Delta u_{n}\right\| e_{1} & \frac{u_{n}}{\left\|\Delta u_{n}\right\|} & -J \frac{u_{n}}{\left\|\Delta u_{n}\right\|}
\end{array}\right)
$$

where $J=\left(\begin{array}{cc}0 & 1 \\ -1 & 0\end{array}\right)$ is the canonical symplectic matrix.

Definition 3.4 (Invariants and normalised invariants) We say the function on $\mathcal{L}_{N}$

$$
I: \mathcal{L}_{N} \rightarrow \mathbb{R}
$$

is a lattice invariant under the action of $G$ if $I(g \cdot \mathcal{L})=I(\mathcal{L})$ for any $g \in G, \mathcal{L} \in \mathcal{L}_{N}$. A local invariant will have the same property in some coordinate chart.

Given a right moving frame $\rho$ on $p$-lattices, we call the invariants

$$
I_{R}=\rho_{R}(\mathcal{L}) \cdot \mathcal{L}
$$

the normalised invariants, where $\rho(\mathcal{L})=\left(\rho_{R}(\mathcal{L})\right)$ with $\rho_{R}(\mathcal{L}) \in G$. Once we choose coordinates in $\mathcal{L}$, given by $\left(u_{J}\right)$, the local invariants are defined to be

$$
I_{R}^{M}=\rho_{R}\left(\left(u_{J}\right)\right) \cdot u_{M}
$$

for $R, M \in \Gamma$. These are clearly invariants of the action.

The normalised invariants generate all other local invariants. In fact, they do generate them even when $R$ is fixed.

Proposition 3.5 If I is any lattice invariant, then I can be written as a function of the normalised invariants $I_{R}^{N}$ for any fixed $R \in \Gamma$.

Proof This is an immediate consequence of the so-called replacement rule. Let $\left(u_{N}\right)$ represent the vertices of the lattice variety. If $I=I\left(\left(u_{N}\right)\right)$ is an invariant of the action, then $I\left(g \cdot\left(u_{N}\right)\right)=I\left(\left(u_{N}\right)\right)$ for all $g \in G$, and in particular for $\rho_{R}\left(\left(u_{J}\right)\right)$. Thus

$$
I\left(\rho_{R}\left(\left(u_{J}\right)\right) \cdot\left(u_{N}\right)\right)=I\left(\left(I_{R}^{N}\right)\right)=I\left(\left(u_{N}\right)\right)
$$

which shows us how to write $I$ in terms of the normalised invariants with $R$ fixed. 
From this wealth of invariants we will be selecting a few, the so-called MaurerCartan invariants. They are the discrete analogue of the invariants defining the classical Frenet-Serret equations and, like their continuous counterpart, together with the set $\left\{I_{R}^{R} \mid R \in \Gamma\right\}$ they will form a generating system.

From now on we will extend the shift operators in the standard way to algebraic functions of $u_{J}$ using the properties $\mathcal{T}_{i}\left(u_{J} u_{R}\right)=\mathcal{T}_{i} u_{J} \mathcal{T}_{i} u_{R}$. We can also apply a shift to $\rho_{J}$ by sending $\rho_{J}$ to $\rho_{J+e_{i}}$ or we could apply it to $\rho_{J}$ by shifting the variables $u_{R}$ that $\rho_{J}$ depends on. But notice that unless the sections in (1) are shifts of each other (i.e. $\mathcal{S}_{R+e_{i}}=\mathcal{T}_{i} S_{R}$ for all $i=1, \ldots p$ and all $R \in \Gamma$ ), these two operations do not need to produce the same result. Given that in many of our situations and in all of our examples we do assume the sections to be invariant under the shifts, we will abuse the notation and denote all these maps by $\mathcal{T}_{i}$, so that, for example, $\mathcal{T}_{i} \rho_{J}=\rho_{J+e_{i}}$.

Definition 3.6 (Maurer-Cartan invariants) Let $\rho$ be a right moving frame along $p$ lattices. We define the right $(R, i)$-Maurer-Cartan group element to be $K_{(R, i)}$, the element of the group given by

$$
K_{(R, i)}=\left(\mathcal{T}_{i} \rho_{R}\right) \rho_{R}^{-1}=\rho_{R+e_{i}} \rho_{R}^{-1} .
$$

Its local coordinates (or the entries of the matrix, if $G \subset \mathrm{GL}(n, \mathbb{R})$ ) will be called the $(R, i)$-Maurer-Cartan invariants.

Definition 3.7 (Diagonal invariants) We denote further the set $\left\{\rho_{R}\left(\left(u_{J}\right)\right) \cdot u_{R}=\right.$ $\left.I_{R}^{R} \mid R \in \Gamma\right\}$ to be the set of diagonal invariants.

Theorem 3.8 Let $\rho$ be any right moving frame. The $(R, i)$ Maurer-Cartan invariants, $i=1, \ldots, p, R \in \Gamma$, together with the diagonal invariants, $\rho_{R}\left(\left(u_{J}\right)\right) \cdot u_{R}=I_{R}^{R}, R \in$ $\Gamma$, generate all other invariants for the action of $G$ on $\mathcal{L}_{N}$.

Proof The proof is based on what are commonly known as the recursion formulae.

Directly from the definitions we get that

$$
K_{(R, i)} \cdot I_{R}^{M}=\left(\mathcal{T}_{i} \rho_{R}\right) \rho_{R}^{-1} \cdot\left(\rho_{R} \cdot u_{M}\right)=\rho_{R+e_{i}} \cdot u_{M}=I_{R+e_{i}}^{M}
$$

and from

$$
K_{(R, i)} \cdot I_{R}^{M}=I_{R+e_{i}}^{M}
$$

we have

$$
I_{R}^{M}=K_{(R, i)}^{-1} \cdot I_{R+e_{i}}^{M} .
$$

Now, since $\Gamma$ is connected, given $M \in \mathbb{Z}^{p}$, any $R \in \mathbb{Z}^{p}$ is related to $M \in \mathbb{Z}^{p}$ through either recurrently increasing or decreasing its individual components, using the shift operator. At each step the invariant obtained when increasing or decreasing the components in $M$ is generated by those in previous steps and by Maurer-Cartan invariants. Thus, we can start using $I_{M}^{M}$ and reach $I_{R}^{M}$, for any $R$, using both versions of the recursion formulas. This proves the statement of the theorem. 
Example 3.9 The simplest example is the translation group viewed as a subgroup of $\mathrm{GL}(n+1, \mathbb{R})$ and acting on $\mathbb{R}^{n}$ as

$$
g \cdot u=\pi\left(\left(\begin{array}{ll}
1 & 0 \\
a & I
\end{array}\right)\left(\begin{array}{l}
1 \\
u
\end{array}\right)\right)=\pi\left(\left(\begin{array}{c}
1 \\
a+u
\end{array}\right)\right)=a+u,
$$

where $\pi$ is the projection in the last $n$ components. If $\left(u_{R}\right)$ is a $p$-lattice in $\mathbb{R}^{n}$, a transverse section to the orbit of the group at $u_{R}$ is given by $u_{R}=0$. Thus, the moving frame is determined by $g \cdot u_{R}=0$, which implies $a=-u_{R}$. The normalised invariants are $I_{R}^{M}=\rho_{R} \cdot u_{M}=u_{M}-u_{R}$, while the Maurer-Cartan matrices are $\rho_{R+e_{i}} \rho_{R}^{-1}$ whose only non-constant entries are the Maurer-Cartan invariants $u_{R}-u_{R+e_{i}}, R \in \Gamma$, $i=1, \ldots, n$. It is straightforward to show that the $I_{R}^{M}$ can be written in terms of the Maurer-Cartan invariants $I_{R}^{R+e_{i}}$. Note that in this example, $I_{R}^{R}=0$ for all $R \in \Gamma$ and they do not contribute to the generating set of invariants.

Example 3.3 cont. In the case of the Euclidean plane, we found a right moving frame given by

$$
\rho_{n}=\left(\begin{array}{cc}
1 & 0 \\
-\Theta_{n} u_{n} & \Theta_{n}
\end{array}\right)
$$

where

$$
\Theta_{n}^{-1}=\left(\frac{u_{n}}{\left\|\Delta u_{n}\right\|}-J \frac{u_{n}}{\left\|\Delta u_{n}\right\|}\right)
$$

The normalised invariants in this case are given by

$$
\rho_{n} \cdot u_{m}=\Theta_{n}\left(u_{m}-u_{n}\right)=\frac{1}{\left\|\Delta u_{n}\right\|}\left(\begin{array}{c}
u_{n} \cdot\left(u_{m}-u_{n}\right) \\
\operatorname{det}\left(u_{n}, u_{m}-u_{n}\right)
\end{array}\right)
$$

for any $n, m$. Notice that $\rho_{n} \cdot u_{n+1}=\left\|\Delta u_{n}\right\| e_{1}$. The Maurer-Cartan matrix is given by

$$
\rho_{n+1} \rho_{n}^{-1}=\left(\begin{array}{cc}
1 & 0 \\
-\Theta_{n+1} \Theta b_{n}+b_{n+1} & \Theta_{n+1} \Theta_{n}^{-1}
\end{array}\right)
$$

where

$$
-\Theta_{n+1} \Theta_{n} b_{n}+b_{n+1}=-\Theta_{n+1}\left(u_{n+1}-u_{n}\right)=\frac{1}{\left\|\Delta u_{n+1}\right\|}\left(\begin{array}{c}
-u_{n+1} \cdot \Delta u_{n} \\
\operatorname{det}\left(u_{n+1}, u_{n}\right)
\end{array}\right)
$$

and

$$
\begin{aligned}
\Theta_{n+1} \Theta_{n}^{-1} & =\frac{1}{\left\|\Delta u_{n+1}\right\|} \frac{1}{\left\|\Delta u_{n}\right\|}\left(\begin{array}{cc}
u_{n+1} \cdot u_{n} & \operatorname{det}\left(u_{n}, u_{n+1}\right) \\
-\operatorname{det}\left(u_{n+1}, u_{n}\right) & u_{n+1} \cdot u_{n}
\end{array}\right) \\
& =\left(\begin{array}{cc}
\cos \alpha_{n} & \sin \alpha_{n} \\
-\sin \alpha_{n} & \cos \alpha_{n}
\end{array}\right),
\end{aligned}
$$

where $\alpha_{n}$ is the angle between $u_{n+1}$ and $u_{n}$. Therefore, a generating set for the MaurerCartan invariants are $\left\|\Delta u_{n}\right\|$ and $\alpha_{n}$, for all $n$. Since the normalised invariants are also 
generated by $\left\|\Delta u_{n}\right\|$ and the angle between $u_{n}$ and $u_{m}$, the Maurer-Cartan invariants generate all the basic ones. Note that, as in the previous example, $I_{R}^{R}=0$ for all $R \in \Gamma$ and they do not contribute to the generating set of invariants.

\subsection{Maurer-Cartan Syzygies}

In this section, we analyse in detail the relationships that can exist among the MaurerCartan invariants.

Remark 3.10 (Syzygies involving the diagonal invariants) In some cases, the additional generating invariants, the "diagonal invariants" $I_{R}^{R}$ (see Definition 3.7) may be non-constant. These invariants obey the trivial recurrence relations, $\mathcal{T}_{i} I_{R}^{R}=$ $\mathcal{T}_{i}\left(\rho_{R} \cdot u_{R}\right)=\left(\mathcal{T}_{i} \rho_{R}\right) \rho_{R+\mathbf{e}_{i}}^{-1} I_{R+\mathbf{e}_{i}}^{R+\mathbf{e}_{i}}=I_{R+\mathbf{e}_{i}}^{R+\mathbf{e}_{i}}$. It can happen that the Maurer-Cartan and the diagonal invariants are not independent of each other, and these dependencies can then be regarded as syzygies between them. Indeed, consider the group $G=(\mathbb{R},+)$ as a scaling action on the positive real line, $\epsilon \cdot u_{n}=\exp (\epsilon) u_{n}$ with the normalisation equation, $\rho_{n} \cdot u_{n+1}=1$. Then $\rho_{n}=-\log u_{n+1}, I_{n}^{n}=u_{n} / u_{n+1}$ and $\rho_{n+1} \cdot \rho_{n}^{-1}=\log I_{n}^{n}$. We conjecture that there are no syzygies involving the diagonal invariants that do not arise from either the trivial recurrence relations between them given above, or those involving the Maurer-Cartan invariants described in this section, together with the dependencies between the diagonal and the Maurer-Cartan invariants.

\subsubsection{Basic Local Syzygies}

From the definition of Maurer-Cartan element, $K_{(N, i)}=\left(\mathcal{T}_{i} \rho_{N}\right) \rho_{N}^{-1}$, we have

$$
\mathcal{T}_{j} K_{(J, i)}=\left(\mathcal{T}_{j} \mathcal{T}_{i} \rho_{J}\right) \mathcal{T}_{j} \rho_{J}^{-1}=\left(\mathcal{T}_{j} \mathcal{T}_{i} \rho_{J}\right) \rho_{J}^{-1} K_{(J, j)}^{-1}
$$

and also

$$
\mathcal{T}_{i} K_{(J, j)}=\left(\mathcal{T}_{i} \mathcal{T}_{j} \rho_{J}\right) \mathcal{T}_{i} \rho_{J}^{-1}=\left(\mathcal{T}_{i} \mathcal{T}_{j} \rho_{J}\right) \rho_{J}^{-1} K_{(J, i)}^{-1}
$$

Given that shifts commute, we obtain

$$
\left(\mathcal{T}_{j} K_{(J, i)}\right) K_{(J, j)}=\left(\mathcal{T}_{i} K_{(J, j)}\right) K_{(J, i)}
$$

This expression gives us a number of algebraic relationships between the different Maurer-Cartan invariants. We will refer to these as basic local syzygies, a discrete generalisation of the differential syzygies that are satisfied by differential invariants, such as the Codazzi-Mainardi equations for Euclidean invariants defined on surfaces. The above syzygies generate most of the possible algebraic relations among MaurerCartan invariants. Further independent syzygies may be created by the topology of the lattice $\mathcal{L}$.

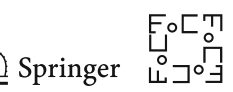


Definition 3.11 We say two syzygies are equivalent up to basic syzygies if one of them is an consequence of the other together with syzygies of the form (6).

To describe global syzygies, we need first to define the discrete fundamental group of the lattice and related material standard in the study of the topology of graphs [48].

\subsubsection{Discrete Fundamental Group}

Let $L$ be a $p$-lattice in $M$, that is, the image of a map from a connected $p$-dimensional subset $\Gamma$ of $\mathbb{Z}^{p}$ to $M$. As before, we will denote the image of $J \in \Gamma$ as $\phi(J)=u_{J} \in M$. For simplicity, let us assume that $\Gamma=\mathbb{Z}^{p}$, although one can apply much of what we will say next to other cases. Notice that, in principle, we are allowing cases when the map $\phi$ is not 1-to- 1 so $L$ does not need to have a trivial topology.

Definition 3.12 (Paths) We say a subset $\gamma \subset L$ is a path of length $r$ joining two points $a, b \in M$, if it can be ordered as $\gamma=\left\{x_{i}\right\}_{i=0}^{r}$, with $x_{0}=a, x_{r}=b$ and $x_{i}$ adjacent to $x_{i+1}$, for all $i=0, \ldots r-1$. We say the path is closed if $a=b$; we say it is simple if $x_{i} \neq x_{j}$ for any $i \neq j$ (except perhaps $x_{0}=x_{r}$ if closed). Notice that by giving the vertices of the path in a certain order we are implicitly assigning an orientation to it. This will be relevant once we associate syzygies to closed paths.

Definition 3.13 (Edge) Given a path $\gamma$ in a lattice, $\gamma=\left\{x_{i}\right\}_{i=0}^{r}$, we say the ordered pair $\left[x_{i}, x_{i+1}\right]$ is an edge of the path. The ordering gives an orientation of the edge.

Definition 3.14 (Sum of paths) Consider the set of all closed paths with base point $a$. One can define the sum of two such paths by concatenation; that is, if $\left\{x_{i}\right\}_{i=0}^{r}$ and $\left\{y_{j}\right\}_{j=0}^{s}$ are two paths, their sum is given by $\left\{x_{i}\right\}+\left\{y_{j}\right\}=\left\{z_{k}\right\}_{k=0}^{r+s+1}$ with

$$
z_{i}=x_{i}, \quad i=0, \ldots, r, \quad z_{r+j+1}=y_{j}, \quad j=0, \ldots, s .
$$

If the paths are not closed, but $x_{r}=y_{0}$, one can equally define the sum of the paths by concatenation.

Definition 3.15 (Basic homotopy) A transformation of a path $\left\{x_{i}\right\}, \psi\left(\left\{x_{i}\right\}\right)=\left\{y_{j}\right\}$ is a basic homotopy if $\left\{y_{j}\right\}$ is equal to $\left\{x_{i}\right\}$ except for

(1) adding or removing a subpath of the form $\left[x_{i}, x_{i+1}\right]+\left[x_{i+1}, x_{i}\right]$;

(2) changing a subpath of the form $\left[x_{i}, x_{i+1}\right]+\left[x_{i+1}, x_{i+2}\right]$ by one of the form $\left[x_{i}, z\right]+\left[z, x_{i+2}\right]$, where $x_{i}, x_{i+1}, x_{i+2}, z$ form a basic square of the lattice.

Transformations (1)-(2) are called the two basic homotopies.

Figure 3 shows examples of basic homotopies.

Definition 3.16 We say the two paths $\left\{x_{i}\right\}_{i=0}^{r}$ and $\left\{y_{j}\right\}_{j=0}^{s}$ joining $a$ and $b$, are homotopically equivalent if $x_{0}=a=y_{0}, x_{r}=b=y_{s}$ and either the paths are equal, or one can be transformed to the other by a finite sequence of basic homotopies.

By construction, homotopy of paths joining $a$ and $b$ is an equivalence relation. Figure 4 shows pairs of homotopic and nonhomotopic paths. 
Fig. 3 Examples of the two basic homotopies applied to the blue paths
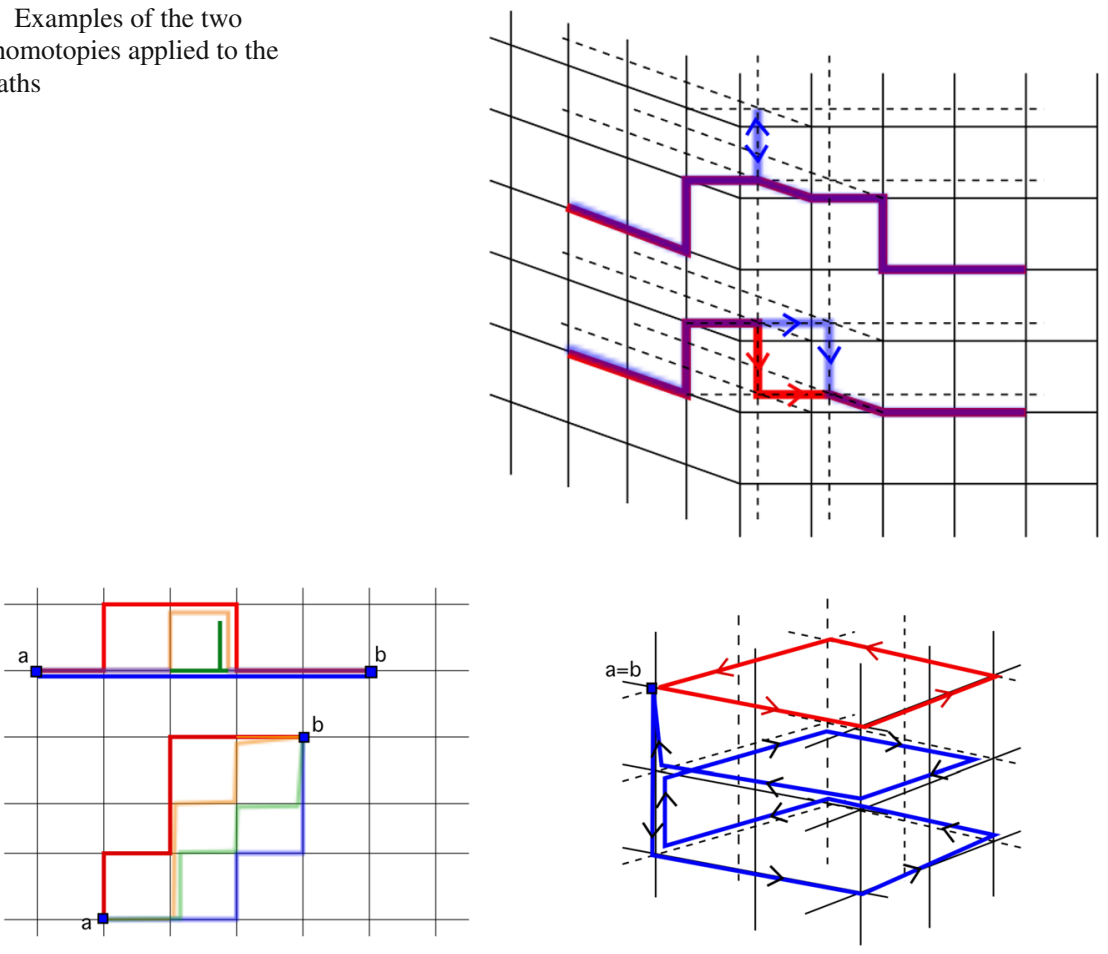

Fig. 4 The red and blue paths on the left are homotopically equivalent, with the yellow and green paths showing the sequence of basic homotopies required, while the ones on the right are not (Color figure online)

Definition 3.17 (Discrete fundamental group) Consider the space of all closed paths based at $a$, and let $\pi_{1}(L, a)$ be the set of homotopy classes of these paths. The operation above endows $\pi_{1}(L, a)$ with a group structure. We call $\pi_{1}(L, a)$ the discrete fundamental group of $L$.

The fundamental group does not depend on the point $a$ chosen, as far as the lattice is connected. (It suffices to join $a$ to a different point $b$ using a path $\gamma$, and use $\gamma$ to relate closed paths based on $a$ to those based on $b$ by conjugation, as done in the continuous case.)

\subsubsection{Syzygies Associated with Closed Paths on a Lattice and Global Syzygies}

Assume we have a moving frame along a path. To each edge of the path we can associate a Maurer-Cartan matrix of invariants as follows:

Assume either that $x_{i}=u_{J}$ and $x_{i+1}=\mathcal{T}_{k} u_{J}=u_{J+e_{k}}$, or that $x_{i+1}=\mathcal{T}_{k}^{-1} u_{J}=$ $u_{J-e_{k}}$. To $\left[x_{i}, x_{i+1}\right]$ we associate the matrix

$$
K_{(J, k)}=\mathcal{T}_{k}\left(\rho_{J}\right) \rho_{J}^{-1}=\rho_{J+e_{k}} \rho_{J}^{-1}
$$

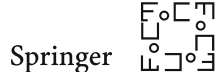


in the first case, and

$$
K_{\left(J-e_{k}, k\right)}^{-1}=\left(\mathcal{T}_{k}\left(\rho_{J-e_{k}}\right) \rho_{J-e_{k}}^{-1}\right)^{-1}=\rho_{J-e_{k}} \rho_{J}^{-1}
$$

in the second case.

We note that the choice of Maurer-Cartan matrix depends on the orientation of the path, with an edge being associated with the inverse matrix if the orientation is reversed.

Next we will associate a group element $K(\gamma)$ to each path $\gamma$ on the lattice, namely, the product of the Maurer-Cartan matrices along the path. From the definition of the Maurer-Cartan matrices in terms of the discrete frame, it will be evident this product telescopes to involve only the discrete frame at the endpoints of the path, provided the discrete frame is defined along the whole path. Nevertheless, in terms of the components of the Maurer-Cartan matrices, the Maurer-Cartan invariants, the product will not telescope-this is the syzygy. Evaluating $K(\gamma)$ for closed paths $\gamma$, leads to relations on the invariants. It becomes important to find those relations which are non-trivial, in the sense that they are not an algebraic consequence of basic local syzygies, given in Eq. (6).

In what follows, we will show that $K(\gamma)$ is a homotopy invariant. Evaluating $K(\gamma)$ on closed paths which are not homotopic to the constant trivial path leads to relations on the Mauer-Cartan invariants which cannot be obtained in terms of the basic local syzygies.

We start with paths which lie in the domain of a discrete frame. This, of course, need not be the case since the existence of moving frames is guaranteed only locally. If we need to cover the lattice with several coordinate patches on which discrete moving frames exist, we will obtain invariant transition matrices associated with the cover of the lattice defined by the domains of the discrete moving frames. We discuss this more involved case later in this section.

Definition 3.18 Assume a discrete frame exists along a path $\gamma$. The product of the Maurer-Cartan matrices along $\gamma$ is denoted $K(\gamma)$. Specifically, for the path $\left\{x_{i}\right\}_{i=0}^{r}$, we have

$$
K\left(\left\{x_{i}\right\}_{i=0}^{i=r}\right)=K\left(\left[x_{r-1}, x_{r}\right]\right) \cdots K\left(\left[x_{0}, x_{1}\right]\right),
$$

where $K\left(\left[x_{i}, x_{i+1}\right]\right)$ is the Maurer-Cartan element associated with the edge $\left[x_{i}, x_{i+1}\right]$ as in (7) and (8) so that $K\left(\left[x_{i}, x_{i+1}\right]\right)=K^{-1}\left(\left[x_{i+1}, x_{i}\right]\right)$. If $\gamma$ is the constant (trivial) path, we define $K(\gamma)=e$.

It is evident that if $\gamma=\gamma_{1}+\gamma_{2}$, then $K(\gamma)=K\left(\gamma_{2}\right) \cdot K\left(\gamma_{1}\right)$. (See Definition 3.14).

In Fig. 5, we illustrate the basic local syzygy, in the form $K\left(\left\{x_{1}, x_{2}, x_{3}, x_{1}\right\}\right)=e$ along a closed path of length four.

Proposition 3.19 Let $\gamma_{1}$ and $\gamma_{2}$ be two paths joining a and $b$ in the lattice L for which a discrete moving frame exists. Assume that $\gamma_{1}$ is homotopic to $\gamma_{2}$, then $K\left(\gamma_{1}\right)=K\left(\gamma_{2}\right)$. 
Fig. 5 In this Figure, we see that along the closed path $\left(x_{1}, x_{2}, x_{3}, x_{4}=x_{1}\right)$ we have $K_{4} K_{3} K_{2} K_{1}=e$, the identity in $G$. This is equivalent to the basic local syzygy, Eq. (6)

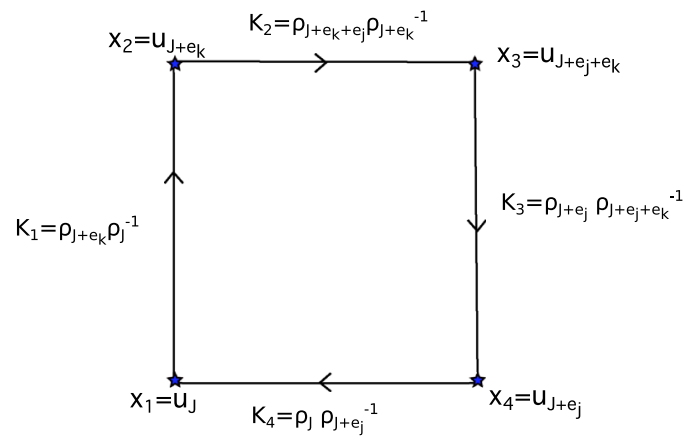

Proof Since a homotopy is a finite composition of basic homotopies, it suffices to show that if $\gamma_{1}$ and $\gamma_{2}$ differ by a basic homotopy, then $K\left(\gamma_{1}\right)=K\left(\gamma_{2}\right)$.

In the case (1), this is trivial since the only difference between $K\left(\gamma_{1}\right)$ and $K\left(\gamma_{2}\right)$ is a product of the form $K_{i} K_{i}^{-1}=e$. In the case (2) it is equally simple since they differ only by a product $K_{i} K_{i+1}$ appearing in $K\left(\gamma_{1}\right)$ and $K_{j} K_{j+1}$ appearing in $K\left(\gamma_{2}\right)$, with $K_{i} K_{i+1}=K_{j} K_{j+1}$ being a local syzygy since their vertices form a square in the lattice.

The discussion thus far lifts naturally to lattice varieties, since adjacency and local shift maps are well defined. The following corollary is an immediate consequence of the previous proposition.

Corollary 3.20 If our lattice variety is covered by one coordinate system and there exists a moving frame defined everywhere, each class $[\gamma]$ of the fundamental group of the lattice defines what we will call a global syzygy of the Maurer-Cartan invariants, in the form $K(\gamma)=e$.

For example, if a discrete frame is defined on an annular lattice $L$, then there will be a path not homotopic to the constant path within $L$, but $K(\gamma)=e$ for all closed paths.

Before turning to consider paths which move through different domains, we note the following.

Important Assumptions We already have the assumption on the coordinate charts $\mathcal{L}_{\alpha}$ which cover our lattice variety, that every edge appears in at least one $\mathcal{L}_{\alpha}$, and so every Maurer-Cartan matrix can be written in (at least one) coordinate system. We assume further that every edge is in a domain of a discrete frame. In this way, every MaurerCartan matrix, every transition matrix, and their local products can be expressed with respect to a single set of coordinates. By taking a refinement of our coordinate cover as necessary, we therefore assume that our cover consists of sets which are domains of both frames and coordinate charts and that every edge appears in at least one element of the cover.

We can associate a group element $K$ to a path moving through different domains, by patching local products of Maurer-Cartan matrices, assuming the local neighbourhoods where the different discrete frames are defined overlap. Overlapping conditions 
are often used to coordinate the geometry in different coordinate domains; in our case, the existence of overlap in the domains of the frames is needed to coordinate the frame on adjacent parts of the path where the domains change. On paths in our lattice varieties, overlapping of domains is guaranteed by the condition that every edge, that is, every pair of adjacent points, lies in at least one of the domains. Indeed, suppose we have two subpaths $\left\{x_{0}, \ldots, x_{i}\right\}$ and $\left\{x_{i+1}, \ldots, x_{j}\right\}$ of a path, and assume we can find a moving frame $\rho_{s}$ at $x_{s}, s=0, \ldots i$ and a different moving frame $\hat{\rho}_{s}$ for $s=i+1, \ldots, j$. Since the edge $\left[x_{i}, x_{i+1}\right]$ must lie in a domain, then at least one of $x_{i}$ or $x_{i+1}$ must lie in both domains, or there is a third domain so that we can split our path into three subpaths, $\left\{x_{0}, \ldots, x_{i}\right\},\left\{x_{i}, x_{i+1}\right\},\left\{x_{i+1}, \ldots, x_{j}\right\}$, each of which lie in the domain of a frame.

So, consider two subpaths $\left\{x_{0}, \ldots, x_{i}\right\}$ and $\left\{x_{i}, x_{i+1}, \ldots, x_{j}\right\}$ of a path, where $x_{i}$ is the guaranteed point of overlap, and where we have a moving frame $\rho_{s}$ at $x_{s}$, $s=0, \ldots i$ and a different moving frame $\hat{\rho}_{s}$ for $s=i, i+1, \ldots, j$. Then we define $M\left(x_{i}\right)=\hat{\rho}_{i} \rho_{i}^{-1}$ so that $\hat{\rho}_{i}=M\left(x_{i}\right) \rho_{i}$. Clearly, since both $\rho_{i}$ and $\hat{\rho}_{i}$ are right equivariant, the matrix $M\left(x_{i}\right)$ is invariant. Then, to the path $\gamma=\left\{x_{0}, \ldots, x_{j}\right\}$ we can associate the product of invariant matrices

$$
\begin{aligned}
K(\gamma) & =\left(\hat{\rho}_{j} \hat{\rho}_{j-1}^{-1}\right)\left(\hat{\rho}_{j-1} \hat{\rho}_{j-2}^{-1}\right) \cdots\left(\hat{\rho}_{i+1} \hat{\rho}_{i}^{-1}\right) M\left(x_{i}\right)\left(\rho_{i} \rho_{i-1}^{-1}\right) \cdots\left(\rho_{1} \rho_{0}\right) \\
& =\widehat{K}_{j-1} \ldots \widehat{K}_{i} M\left(x_{i}\right) K_{i-1} \ldots K_{0}
\end{aligned}
$$

with the invariant matrix $M\left(x_{i}\right)$ linking the Maurer-Cartan matrix in one coordinate system to the next.

Definition 3.21 If a vertex $x$ lies in the domains of both the discrete frame $\rho$ and the discrete frame $\widehat{\rho}$, we say the group element

$$
M(x ; \widehat{\rho}, \rho)=\widehat{\rho}(x) \rho(x)^{-1}
$$

is the transition Maurer-Cartan matrix at the vertex $x$, associated with the change of frame from $\rho$ to $\widehat{\rho}$.

If a vertex lies in the intersection of several frame domains, there will be co-cycle conditions. For example, if $x \in \operatorname{dom}\left(\rho_{\alpha}\right) \cap \operatorname{dom}\left(\rho_{\beta}\right) \cap \operatorname{dom}\left(\rho_{\delta}\right)$, then clearly

$$
M\left(x ; \rho_{\delta}, \rho_{\beta}\right) M\left(x ; \rho_{\beta}, \rho_{\alpha}\right) M\left(x ; \rho_{\alpha}, \rho_{\delta}\right)=e .
$$

Two equal closed paths can have different group elements $K(\gamma)$ if the choices of either the initial or the final moving frames are different. Thus, our element of the group depends not only on $\gamma$ but also on the initial and final choice of moving frame. In this case we will denote the group element above

$$
K(\gamma ; \alpha, \beta)
$$

for the element of the group that starts in $\operatorname{dom}\left(\rho_{\alpha}\right)$ and ends in $\operatorname{dom}\left(\rho_{\beta}\right)$, and where these are the choices of frame for the calculation of the initial and final Maurer-Cartan matrices. By analogy, we will also denote by $M(x ; \alpha, \beta)$ the matrix $M\left(x ; \rho_{\alpha}, \rho_{\beta}\right)$. 
Fig. 6 Several ways a path can move through an interchange

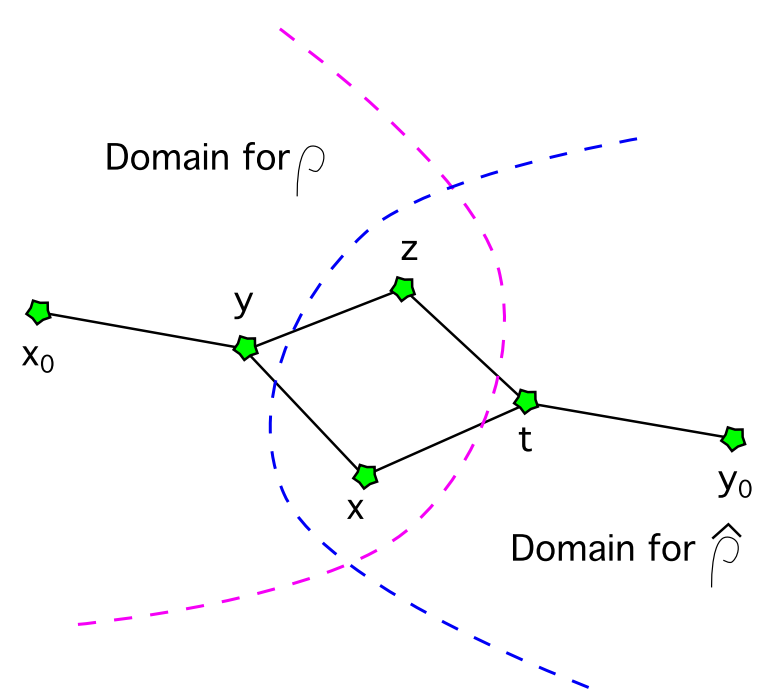

Lemma 3.22 $K(\gamma ; \alpha, \beta)$ does not depend on the choice of coordinates or moving frames one chooses along $\gamma$, only on the initial and final ones.

Proof Assume at some point $x$ we make different choices of moving frame at a point $x$ in the overlap of (at least two) different frame domains, so we move from $\rho_{\mu}$ to $\rho_{\eta}$ for one path and from $\rho_{\mu}$ to $\rho_{\nu}$ for the other. In that case we introduce the transition factor $M(x ; \eta, \mu)$ in one of the lifts, and $M(x ; \nu, \mu)$ in the other one, and we continue the different paths using the corresponding choices. At some point we need to come back to a common choice, even if that happens only at the end of the path. But when we expand the different factors of $K(\gamma ; \alpha, \beta)$ in terms of moving frames, the intermediate factors all vanish as we saw before, and the difference is only at the beginning and at the end of the product. Thus, without losing generality we can assume that we come back to a common moving frame right after we introduce the split.

That is, a path includes the factor

$$
M(x ; \beta, \eta) M(x ; \eta, \mu)
$$

while the other includes

$$
M(x ; \beta, v) M(x ; v, \mu) .
$$

But using (10) we have that both these factors are equal to $M(x ; \beta, \mu)$, and hence they are equal.

We now argue that $K(\gamma ; \alpha, \beta)$ is still a homotopy invariant for paths that start and end with the $\alpha$ and $\beta$ choice of moving frame, even if the path moves through changing domains of discrete frames (and changing coordinate systems). Consider Fig. 6 , in which we assume that $[x, y],[y, z]$ are edges in the domain of $\rho$, while $[x, t]$ and $[t, z]$ are edges in the domain of $\widehat{\rho}$.

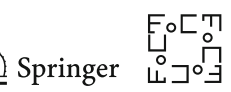


We consider paths $\gamma_{i}$ running from $x_{0}$, in the domain of $\rho$, to $y_{0}$ in the domain of $\widehat{\rho}$. If $\gamma_{1}$ goes via $x$ and $t$, then we must switch from $\rho$ to $\widehat{\rho}$ at $x$. We achieve this by using the matrix $M(x)=\widehat{\rho}(x) \rho(x)^{-1}$ (which is invariant as the frames are right frames), and then defining $K\left(\gamma_{1}\right)$ to be

$$
\begin{aligned}
K\left(\gamma_{1}\right) & =\widehat{K}\left(\left[t, y_{0}\right]\right) \widehat{K}([x, t]) M(x) K([y, x]) K\left(\left[x_{0}, y\right]\right) \\
& =\widehat{\rho}\left(y_{0}\right) \widehat{\rho}(t)^{-1}\left(\widehat{\rho}(t) \widehat{\rho}(x)^{-1}\right)\left(\widehat{\rho}(x) \rho(x)^{-1}\right) \rho(x) \rho^{-1}(y) \rho(y) \rho\left(x_{0}\right)^{-1} \\
& =\widehat{\rho}\left(y_{0}\right) \rho\left(x_{0}\right)^{-1} .
\end{aligned}
$$

Considering the path $\gamma_{2}$ from $x_{0}$ to $y_{0}$ via $z$ results in

$$
K\left(\gamma_{2}\right)=\widehat{K}\left(\left[t, y_{0}\right]\right) \widehat{K}([z, t]) M(z) K([y, z]) K\left(\left[x_{0}, y\right]\right)
$$

which is also equal to $\widehat{\rho}\left(y_{0}\right) \rho\left(x_{0}\right)^{-1}$ by a similar argument, and thus we have

$$
K\left(\gamma_{1}\right)=K\left(\gamma_{2}\right)
$$

Since we have homotopy invariance of the $K$ element within domains, in this way we can see that even passing through a change of domain, we maintain homotopy invariance.

In order to prove homotopy invariance in general, we construct a "lift" $\overline{\mathcal{L}}$, of $\mathcal{L}$, with respect to a cover, satisfying our assumptions, of $\mathcal{L}$. We can use this lift to keep track of which discrete frame we are using at each point on our paths in $\mathcal{L}$. The lift $\overline{\mathcal{L}}$ that we construct is not a lattice variety in general, and does not lie in $M$, but nevertheless serves our purpose here. To construct $\overline{\mathcal{L}}$, we take the disjoint union of the charts, together with their edges (adjacencies), and for every $x \in \mathcal{L}_{\alpha} \cap \mathcal{L}_{\beta}$, we take a new adjacency, or edge, $\left[\left.x\right|_{\alpha},\left.x\right|_{\beta}\right]$, and let this be a new edge in $\overline{\mathcal{L}}$, with the associated Maurer-Cartan element being $\rho_{\beta} \rho_{\alpha}^{-1}$, the transition Maurer-Cartan matrix. See Fig. 7 , which shows the lifting for the case of Fig. 6. Define the projection from $\overline{\mathcal{L}}$ to $\mathcal{L}$ as the natural projection that collapses the different copies of the vertices which lie in intersections of charts. That is

$$
\pi: \overline{\mathcal{L}} \rightarrow \mathcal{L}, \quad \pi\left(x_{i}\right)= \begin{cases}x_{i} & \text { if } x_{i} \text { belongs only to one domain } \\ x & \text { if } x_{i}=x_{\alpha} \text { where } x_{\alpha} \text { is a lift of } x \in \mathcal{L}_{\alpha}\end{cases}
$$

A path $\bar{\gamma}$ in $\overline{\mathcal{L}}$ is a lift of a path in $\mathcal{L}$ when the projection of $\bar{\gamma}$ is $\gamma$. Lifts may not be unique, as they depend, for example, on the element of $\pi^{-1}(a)$ at which the path begins, where $a$ is the initial vertex of $\gamma$.

Changing from one frame to another along a path is, in $\overline{\mathcal{L}}$, simply proceeding from one vertex to another, with the transition Maurer-Cartan elements, Definition 3.21, being the group element associated with the new edge. We note that a change of coordinates simply changes the local labelling of the points, and so is less important when considering the group element associated with a path. 
Fig. 7 The situation of Fig. 6 translated to $\overline{\mathcal{L}}$, the "lift" of $\mathcal{L}$

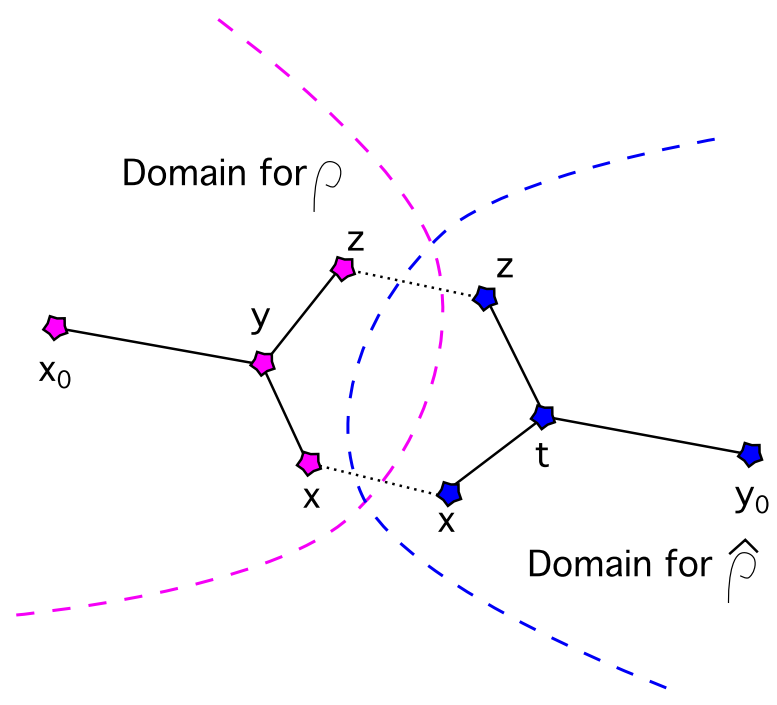

We now show that in $\overline{\mathcal{L}}$ we have new basic local syzygies obtained from the transition Maurer-Cartan matrices. These new local syzygies allow us to obtain a result similar to that of Proposition 3.19, but for the more general situation where we need to change domains of our moving frames. Consider Fig. 8. Let the domain of $\rho$ be $\mathcal{L}_{\alpha}$ and the domain of $\widehat{\rho}$ be $\mathcal{L}_{\beta}$. Consider the simple closed path $\gamma=$ $\left\{\left.x\right|_{\alpha},\left.x\right|_{\beta},\left.y\right|_{\beta},\left.y\right|_{\alpha},\left.x\right|_{\alpha}\right\}$. If $K\left(\left[y_{\beta}, x_{\beta}\right]\right)=\widehat{\rho}(y) \widehat{\rho}(x)^{-1}, K\left(\left[x_{\beta}, x_{\alpha}\right]\right)=\widehat{\rho}(x) \rho(x)^{-1}$, $K\left(\left[y_{\beta}, y_{\alpha}\right]\right)=\widehat{\rho}(y) \rho(y)^{-1}, K\left(\left[y_{\alpha}, x_{\alpha}\right]\right)=\rho(y) \rho(x)^{-1}$, the $K$ element for this path is,

$$
K(\gamma)=\left(\rho(x)^{-1} \rho(y)\right)\left(\rho(y) \widehat{\rho}(y)^{-1}\right)\left(\widehat{\rho}(y) \widehat{\rho}(x)^{-1}\right)\left(\widehat{\rho}(x) \rho(x)^{-1}\right)=e
$$

showing this path defines a basic syzygy in $\overline{\mathcal{L}}$.

Finally, we define the monodromy of a closed path in $\mathcal{L}$. When such a path is lifted to $\overline{\mathcal{L}}$ it need not be closed, as it may begin in one frame domain and return in another. Consider the lift $\bar{\gamma}$ of a path $\gamma$ beginning at $x_{0}$ in the domain of the frame $\rho_{\alpha}$, and ending at $x_{0}$ in the domain of the frame $\rho_{\beta}$. We can close the path in $\overline{\mathcal{L}}$, by adding to $\bar{\gamma}$, the edge $\left[\left.x_{0}\right|_{\beta},\left.x_{0}\right|_{\alpha}\right]$, but in principle this need not happen. Therefore, the element of the group associated with the lift would not be equal to $e$, but rather to $M(x ; \alpha, \beta)$ (or its inverse, depending on the orientation). We call this group element the monodromy of the lift.

We can now state the more general theorem concerning homotopy invariance.

Theorem 3.23 To each path $\gamma$ in the lattice variety $\mathcal{L}$, and to each choice of initial and final moving frame, we can associate a group element, $K(\gamma, \alpha, \beta)$, such that if $\gamma_{1}$ and $\gamma_{2}$ are homotopic in $\mathcal{L}$, then $K\left(\gamma_{1}, \alpha, \beta\right)=K\left(\gamma_{2}, \alpha, \beta\right)$. 

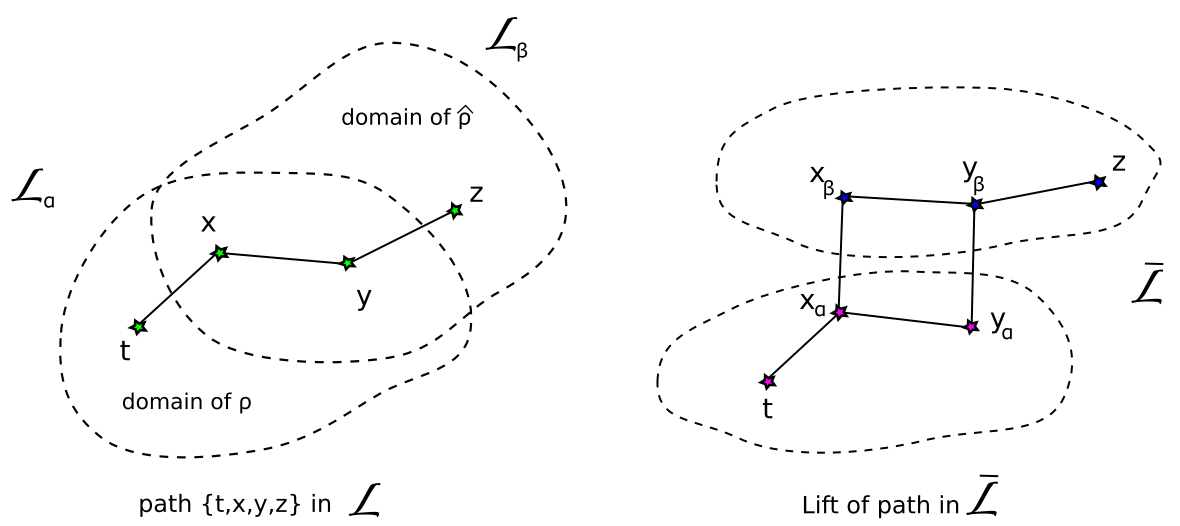

Fig. 8 New edges in $\overline{\mathcal{L}}$ give rise to new local syzygies, here, $K\left(\left[y_{\beta}, x_{\beta}\right]\right) K\left(\left[x_{\beta}, x_{\alpha}\right]\right)=K\left(\left[y_{\beta}, y_{\alpha}\right]\right)$ $K\left(\left[y_{\alpha}, x_{\alpha}\right]\right)$

Proof We first note that $\overline{\mathcal{L}}$ has essentially a global moving frame, in the sense that every vertex has an equivariant group element associated, namely $\rho_{\alpha}$ for $x_{\alpha}$ and $\rho_{\beta}$ for $x_{\beta}$. Hence we can define $K(\gamma)$ for any path, the same way we did previously.

We first note that if $\{\bar{a}\}$ is any closed path lifting the trivial path $\{a\}$ to $\overline{\mathcal{L}}$, then the co-cycle conditions (10) are given by $K(\bar{a})=e$. When we lift a path $\gamma$ to $\overline{\mathcal{L}}$, we need to keep track of which discrete frame we are working in, but as we saw before, the element $K(\gamma ; \alpha, \beta)$ is affected only by the beginning and end choices.

Assume two paths are homotopically equivalent, and let us lift the homotopy. By construction of $\overline{\mathcal{L}}$, we can assume the endpoints of the lift of the homotopic paths also remain fixed and determined by the $\alpha$ and $\beta$ choices. As in the proof of Proposition 3.19 , it will suffice if we show that two paths that differ by one of the basic homotopies have the same $K(\gamma ; \alpha, \beta)$, even if we need to change the moving frame domain. But this was already proved in the argument concerning Eq. (11).

The syzygy of any closed path $\gamma$, where the domain of the discrete frames are considered to be the same at the end and at the beginning, is $K(\gamma ; \alpha, \alpha)=K(\gamma)=e$, while those where $\alpha \neq \beta$ will have a non-trivial monodromy $K(\gamma ; \alpha, \beta)=M \neq e$. Furthermore, different choices of $\alpha$ give rise to the same syzygy: the group elements are related by conjugation

$$
K(\gamma ; \alpha, \alpha)=\rho_{\alpha} \rho_{\beta}^{-1} K(\gamma ; \beta, \beta) \rho_{\beta} \rho_{\alpha}^{-1},
$$

and the transition matrices $\rho_{\alpha} \rho_{\beta}^{-1}$ are essentially a change of coordinates. Indeed, a frame defines a local coordinate system of the form $U \times \mathcal{K}$ where $U$ is a neighbourhood of the identity in $G$ and $\mathcal{K}$ is the cross section which has invariants for coordinates [38, Chapter 4]. We thus have the following corollary.

Corollary 3.24 Let $[\gamma]$ be the homotopy class of a closed path $\gamma$ in $\mathcal{L}$. Each element $[\gamma]$ of $\pi_{1}(\mathcal{L})$ gives rise to a syzygy on the Maurer-Cartan invariants, in the form $K(\gamma)=e$. 
Fig. 9 Discrete toroidal lattice variety, with periodicities $T_{1}=8$ and $T_{2}=6$ (Color figure online)

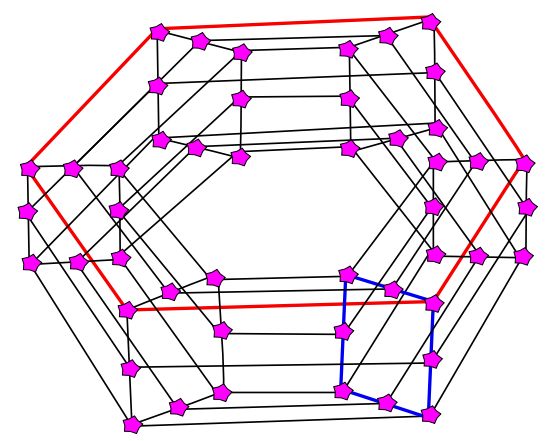

Notice that associating a syzygy to a closed path with a monodromy is essentially the same as associating a syzygy to the closed path for which the beginning and end moving frames are the same. Indeed, $K(\gamma ; \alpha, \alpha)=K(\gamma, \alpha, \beta) M(x ; \beta, \alpha)$, and hence $K(\gamma ; \alpha, \alpha)=e$ is the same syzygy as $K(\gamma ; \alpha, \beta)=M(x ; \alpha, \beta)$.

Example 3.25 Consider a bi-periodic lattice $L=\left\{z_{n, m}\right\}_{n, m \in \mathbb{Z}}$ with $z_{n+k T_{1}, m}=z_{n, m}$ and $z_{n, m+k T_{2}}=z_{n, m}$ for some periods $T_{1}, T_{2} \in \mathbb{Z}$. For simplicity, assume we can find a global moving frame $\rho=\left(\rho_{n, m}\right)$. The topology of this lattice is comparable to that of a torus, and one can easily show that

$$
\pi_{1}(L)=\mathbb{Z}^{2}
$$

The two generators of $\pi_{1}(L)$ (marked in Fig. 9 with different colours) are represented by the two global syzygies

$$
K_{0, m} K_{1, m} \ldots K_{T_{1}-1, m}=e, \quad K_{n, 0} K_{n, 1} \ldots K_{n, T_{2}-1}=e .
$$

If a global moving frame does not exist, then the product might be equal to a monodromy matrix that will depend on the choice of moving frame at the beginning and the end of the closed path. If we choose the same moving frame, then the syzygy will be independent of the point chosen as beginning and end, and it will be as above.

\section{Continuous Limits of the Discrete Picture: Lattice-Based Multispace}

In this section we show how one can construct a continuous moving frame embedded in a smooth family of discrete frames by coordinating the transverse sections that determine them in a way that guarantees the convergence of the discrete family to the continuous one. This is achieved using our lattice-based multispace, in which derivatives and their finite difference approximations exist in a single manifold containing both the jet bundle and Cartesian products of the base space. Both smooth and discrete frames are then part of a single frame on this multispace, and their relationship is given by the continuity of the multispace frame under coalescence. We show in this case that not only moving frames but also discrete invariants and local discrete syzygies

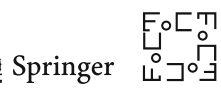


converge to differential invariants and differential syzygies respectively. We will use our multispace constructions to describe discretisations of integrable systems, and to finite difference models of variational systems, in Sect. 5. In the Appendix, we will show that more generally, an equicontinuous family of discrete frames will converge to a smooth frame.

First of all, we recall the definition of "multispace for curves", as developed by Olver in [51]. Olver provides coordinates for his space of pointed curves in terms of the Lagrange approximation of the curve via interpolation at specific (given) points. The coordination of the discrete and the smooth pictures is a consequence of the fact that Lagrange interpolation becomes Hermite interpolation under coalescence. In order to provide coordinates for our higher-dimensional generalisation of Olver's construction, we need to restrict our 'pointed surfaces' to those where the Lagrange and Hermite interpolations are similarly related and to where the interpolations vary in a smooth manner with respect to the data. The details of the interpolation are critical, since the coefficients of the interpolation polynomial will define the desired coordinates. We use the theory of multivariate polynomial approximation due to de Boor and Ron, [11-13], described in Sect. 4.2. We then describe our lattice-based multispace and prove that it is a manifold. In fact, we detail two related versions of multispace, one containing the jet bundle $J(M, \mathbb{R})$ and one containing the jet bundle $J(U, M)$ where $U \subset \mathbb{R}^{p}$ for any $p \leq \operatorname{dim} M$. Both arise in the applications. Thereafter we show that the limit of the discrete Maurer-Cartan matrices are the smooth Maurer-Cartan matrices and that the local syzygies (Eq. 6) limit to the so-called zero curvature condition of the smooth Maurer-Cartan matrices, in Sect. 4.4. We also describe mixed discrete/continuous cases.

\subsection{Olver's Multispace for Curves}

The idea behind the definition of multispace is to create a manifold where both discrete and continuous cases coexist in one overarching smooth construction, where the continuous frame is a limit of the discrete, and the limit of the discrete data is the continuous data. Multispace resembles the jet spaces, but includes also discrete versions of the jet spaces.

Given a manifold $M$, define the $n$th jet space of $M$ at $p \in M$, and denote it by $J_{p}^{n}$, to be the equivalence class of submanifolds of $M$ with order of contact $n$ at $p$. The jet bundle is defined as

$$
J^{n}(M)=\cup_{p \in M} J_{p}^{n},
$$

with the standard bundle structure. We let $C^{(n)}=C^{(n)}(M)$ denote the set of all $(n+1)$ pointed curves contained in $M$; that is, the set of $\left(z_{0}, \ldots, z_{n} ; C\right)$, where $C$ is a curve and $z_{i}$ are $n+1$ points in $C$, not necessarily distinct. We denote by

$$
n_{i}=\#\left\{j \mid z_{j}=z_{i}\right\}
$$

the number of points that coincide with $z_{i}$. 
Definition 4.1 (Multispace for curves) Let $\mathbf{C}$ and $\widetilde{\mathbf{C}}$ be two $(n+1)$-pointed curves

$$
\mathbf{C}=\left(z_{0}, \ldots, z_{n} ; C\right) ; \quad \widetilde{\mathbf{C}}=\left(\widetilde{z}_{0}, \ldots, \widetilde{z}_{n} ; \widetilde{C}\right)
$$

The distinguished points can coincide. We say that $\mathbf{C}$ and $\widetilde{\mathbf{C}}$ have $n$th order multicontact if, and only if there exists a permutation $\pi:\{0,1, \ldots, n\} \rightarrow\{0,1, \ldots, n\}$ such that

$$
z_{i}=\widetilde{z}_{\pi(i)}, \quad \text { and }\left.\quad j_{n_{i}-1} C\right|_{z_{i}}=\left.j_{n_{i}-1} \widetilde{C}\right|_{z_{\pi(i)}}, \quad \text { for each } i=0, \ldots, n,
$$

where $j_{k} C$ denotes the $k$ th jet of the curve $C$.

The $n$ th-order multispace, denoted $M^{(n)}$, is the set of equivalence classes of $(n+1)$ pointed curves in $M$ under the equivalence relation of $n$ th-order multicontact. The equivalence class of an $(n+1)$-pointed curve $\mathbf{C}$ is called its $n$ th-order multijet and is denoted by $\mathbf{j}_{n} \mathbf{C} \in M^{(n)}$.

When all the points are distinct, then two curves belong to the same equivalence class whenever they have the distinguished points $z_{i}$ in common. Thus, we can identify this special subset with the off-diagonal Cartesian product, denoted by $M^{\diamond(n+1)}$ in [51]. On the other hand, if all the points coincide, then the class is equal to the jet class. Thus, both extremes can be found in one space, together with all the intermediate cases. In the first part of [51] the main result is the following theorem.

Theorem 4.2 If $M$ is a smooth $m$-dimensional manifold, then its $n$th order multispace $M^{(n)}$ is a smooth manifold of dimension $(n+1) m$, which contains the off-diagonal part $M^{\diamond(n+1)}$ of the Cartesian product space as an open, dense submanifold, and the nth order jet bundle $J^{n}(M)$ as a smooth submanifold.

The topology is inherited from that of the manifold $M$, and the proof is based on finding coordinate systems in a neighbourhood of an equivalence class $\mathbf{j}_{n} \mathbf{C}$. The coordinate system is given by the classical divided differences and their limits. That is, given a curve $C$ with $n+1$ distinguished points $\left\{z_{0}, \ldots, z_{n}\right\}$ and with a certain order of contact $n_{i}-1$ at each point, there exists a unique polynomial $p$ of degree $n$ such that $p\left(z_{i}\right)=C\left(z_{i}\right)$ and such that $p^{(k)}\left(z_{i}\right)=C^{(k)}\left(z_{i}\right)$ for any $k \leq n_{i}$ and any $i=0, \ldots, n$. The polynomial is a natural representative of the class $\mathbf{C}$ and its coefficients provide smooth coordinates in a neighbourhood of $\mathbf{C}$. Of particular importance is that the coordinates are smooth under the coalescence of points $z_{i}$. For more details, see [51].

In the second part of the paper [51], the author assumes there is a Lie group $G$ acting on the manifold $M$, and he defines the action of this group on the multispace as that naturally induced by it: the action on the differential part is the prolonged one, and explicit formulae for the action of the group on classical divided differences are given. He also explains how, assuming that one chooses a cross section to the orbit of the group at a point $\mathbf{C}$, and requiring the local cross section to be transverse also to the jet space (thus defining a cross section for the prolonged action on $J^{n}$ ); then one can find a moving frame for the action of the group on the multispace with the desired property, that is, the resulting moving frame will be the standard continuous moving frame when restricted to jets, and the discrete one when restricted to $M^{\diamond(n+1)}$. The overarching

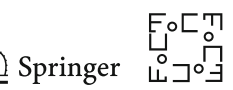



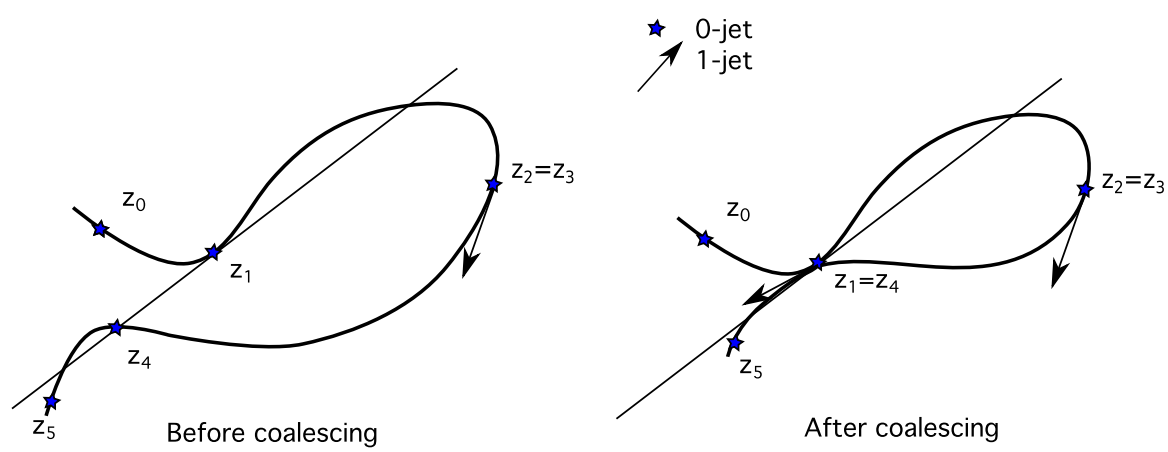

Fig. 10 Coalescence of distinguished points in a pointed curve does not require the points to be adjacent, in some sense, on the curve. Here the coalescence takes place along the straight line. Note there needs to be a well-defined tangent of $C$ at the point of coalescence

continuity of the multispace manifold guarantees that one is the continuous limit of the other as the points coalesce.

We note several features of Olver's multispace for pointed curves:

(i) the curve $\mathbf{C}$ is not parametrised and the points $z_{i}$ on the curve need not be labelled in order with respect to some parametrisation,

(ii) coalescence can take place between any two of the $z_{i}$ on the curve, see Fig. 10,

(iii) none of the $z_{i}$ are distinguished in the sense that one of them is a natural base point for a projection of the multispace to $M$,

(iv) the pointed curve $\mathbf{j}_{n} \mathbf{C}$ is essentially a set of points with a contact condition at each point.

In our construction of a higher-dimensional lattice-based multispace, and hence its restriction to a single variable, only a version of property (iv) remains.

Our next section describes an interpolation scheme which can be applied to our geometric construction.

\subsection{Multivariate Interpolation}

One of the main problems with multivariate interpolation is that the solution to the interpolation problem is not unique in general and it might not even exist; a wellknown theorem describing this phenomena is the Mairhuber-Curtis Theorem [60]. For example, if we fix the values of a function $f(x, y)$ at the two points $(1,2),(-1,1)$ and we want to find a polynomial in $x$ and $y$ of minimum order, such that it coincides with the function at those points, we can use $f(x, y)=a+b x$ or $f(x, y)=a+b y$, and there is no reason why we would chose one over the other. On the other hand, if we fix the value of the function at $(1,2)$ and $(1,-1)$, then the first choice is not appropriate unless the function has the same value at both points, while the second one works. Thus, the choice of interpolating polynomial might depend on the data, it might not be unique, or even exist, and sometimes there is no reason to favour one choice over a different one. In the $p=1$ interpolation case none of these problems exist. 
Thus, a main question in multivariate interpolation is: is there a family of polynomials which can interpolate the values of a given function and which has properties like generality, minimal degree, uniqueness and having a well-defined Hermite polynomial (one for which not only the values of the polynomial, but also its derivatives at the points coincide with those of the function) as points coalesce? These are the properties we will need if we want to use them to define smooth coordinates in our multispace. This question was answered by de Boor and Ron in [11-13]; we describe below their solution to the interpolation problem as it applies to our particular case.

Interpolation Notation Let $\Pi$ be the set of $p$-variate polynomials, and $\Pi^{\prime}$ its algebraic dual. Let $\Lambda$ be a subspace of $\Pi^{\prime}$. We will denote by $\Pi_{k}$ the subset of polynomials of degree less than or equal to $k$.

Definition 4.3 We say that $P \subset \Pi$ is correct for $\Lambda$ if for any continuous linear functional $F$ on $\Lambda$, there exist a unique $q \in P$ such that

$$
F(\lambda)=\lambda(q)
$$

for any $\lambda \in \Lambda$. We also say $P$ interpolates $\Lambda$.

The dual space $\Pi^{\prime}$ can be identified with functions analytic at the origin $o$, using the bilinear form

$$
\langle f, q\rangle=\sum_{J \in \mathbb{Z}_{+}^{p}} \frac{D^{J} f(o) D^{J} q(o)}{J !},
$$

where $q$ is a $p$-variate polynomial and $f$ is a function analytic at the origin. One can also use formal power expansions at the origin instead of analytic functions in the obvious way, without too much trouble. See $[11,12]$ for more details.

Example 4.4 (Lagrange Interpolation) If $\Lambda$ is spanned by point-evaluations, $\Lambda=$ $\left\langle\lambda_{\theta}\right\rangle_{\theta \in \Theta}$ with $\lambda_{\theta}(p)=p(\theta), \theta \in \mathbb{R}^{p}$, finding $P$ correct for $\Lambda$ solves a Lagrange interpolation problem. Indeed, if $\Theta$ represents a finite number of points in the parameter space $D \subset \mathbb{R}^{p}$, and $\lambda_{\theta}$ is evaluation at an element of $\Theta$, then one can check that the power series representing $\lambda_{\theta}$ is the Taylor expansion of $e^{\theta \cdot \mathbf{x}}$ (see [11,12]). An analytic function $F$ defines a continuous linear functional on $\Lambda$ via (12) and $F\left(\lambda_{\theta}\right)=$ $\left\langle F, e^{\theta \cdot \mathbf{x}}\right\rangle=F(\theta)$. Thus, $P$ is correct if for any $F$ there exists $p \in P$ such that

$$
F\left(\lambda_{\theta}\right)=F(\theta)=\lambda_{\theta}(p)=p(\theta)
$$

for all $\theta \in \Theta$, which is the definition of Lagrange interpolation, see Example 4.9.

Example 4.5 (Hermite Interpolation) If we choose instead the set

$$
\Lambda=\left\{\lambda_{q, \theta}\right\}_{\theta \in \Theta, q \in V \subset \Pi}
$$

where

$$
\lambda_{q, \theta}(p)=\lambda_{\theta}(q(\nabla)(p))=q(\nabla)(p)(\theta)
$$

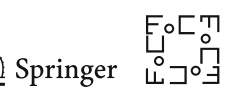


and where $\nabla$ is the gradient vector and $q \in V \subset \Pi$ is a properly chosen polynomial with coefficients reflecting the differential data we need to match (for example, $q(x, y)=x^{2}$ if we wish to match the second derivative in $\left.x\right)$, then the associated power series representing $\lambda_{q, \theta}$ is $q(\mathbf{x}) e^{\theta \cdot \mathbf{x}}$ (see $[11,12]$ ). Therefore, finding $P$ correct for $\Lambda$ is equivalent to finding a family of polynomials $P$ such that for any linear functional on $\Lambda$ represented by an analytic function $F$, there exists a unique polynomial $p \in P$ with the property

$$
F\left(\lambda_{q, \theta}\right)=\left\langle F, q(\mathbf{x}) e^{\theta \cdot \mathbf{x}}\right\rangle=q(\nabla)(F)(\theta)=\lambda_{q, \theta}(p)=q(\nabla)(p)(\theta)
$$

for all $\theta \in \Theta$ and all $q \in V$. The different choices of $V$ allow us to find unique polynomials that coincide with $F$ and different choices of derivatives, or combinations of derivatives, on $\Theta$. This is the solution to the Hermite interpolation problem, see Example 4.10.

Definition 4.6 Given a formal power series at $x=0$, call it $f$, we denote by $f_{\downarrow}$ the homogeneous term in the power expansion of $f$ of lowest order.

For example, if $\theta, \mathbf{x} \in \mathbb{R}^{p}, e_{\downarrow}^{\theta \cdot \mathbf{x}}=1$.

Definition 4.7 Given a finite subset $\Lambda \subset \Pi^{\prime}$, we can identify each of its elements with formal power series at the origin using (12), and we can consider $\widehat{\Lambda}$ to be the vector space spanned by $\Lambda$ as represented by these series. Define the vector space

$$
\Lambda_{\downarrow}:=\operatorname{span}\left\{f_{\downarrow}, f \in \widehat{\Lambda}\right\} .
$$

For example, if we consider the planar case, and $\Theta=\{(1,-1),(0,2)\}$, then $e^{(1,-1) \cdot \mathbf{x}}=1+x-y+\cdots$ and $e^{(0,2) \cdot \mathbf{x}}=1+2 y+\cdots$. Since $e^{(1,-1) \cdot \mathbf{x}}-e^{(0,2) \cdot \mathbf{x}}=$ $x-3 y+\cdots$, we have that $\Lambda_{\downarrow}$ is the linear space spanned by the polynomials $p_{1}(x, y)=1=e_{\downarrow}^{(1,-1) \cdot \mathbf{x}}$ and $p_{2}(x, y)=x-3 y=\left(e^{(1,-1) \cdot \mathbf{x}}-e^{(0,2) \cdot \mathbf{x}}\right)_{\downarrow}$.

From now on, if $\Theta$ is a data set, we say that $\Lambda_{\downarrow}$ is continuous on $\Theta$ if whenever the data $\Theta^{\prime}$ is close to $\Theta$ in the standard product topology, then $\Lambda_{\downarrow}^{\prime}$ is close to $\Lambda_{\downarrow}$ in the standard topology of polynomial spaces.

The following theorem is a compilation of results found in [13]. Since our constructions are lattice based, we assume that $\Lambda$ is defined as in the Lagrangian interpolation associated with a lattice of points $\Theta \subset \mathbb{R}^{p}$, in which case we also write $\Lambda$ as $\Lambda^{\Theta}$. We omit the superscript $\Theta$ where the dependence of $\Lambda$ on $\Theta$ is clear.

Theorem 4.8 [13] The space $\Lambda_{\downarrow}$ has the following properties:

(1) Well defined. For any finite $\Theta$, the assignment $\Lambda \rightarrow \Lambda_{\downarrow}$ exists, is unique and $\Lambda_{\downarrow}$ is correct for $\Lambda$.

(2) Continuity. Recall that $\Pi_{k}$ is the set of p-variate polynomials of degree less or equal to $k$. If $\Pi_{k} \subseteq \Lambda_{\downarrow} \subseteq \Pi_{k+1}$, then the assignment $\Lambda \rightarrow \Lambda_{\downarrow}$ is continuous with respect to $\Theta$.

(3) Coalescence $\Longrightarrow$ Osculation. That is, the Lagrange interpolation becomes the Hermite interpolation under coalescence, provided the coalescence is well controlled, so that data points coalesce along embedded curves. 
(4) $\Lambda_{\downarrow}$ is closed under differentiation and it is spanned by a homogeneous basis.

(5) Minimal degree. $\Lambda_{\downarrow}$ has minimal degree.

(6) Monotonicity. If $\Theta \subset \Theta^{\prime}$, then $\Lambda_{\downarrow}^{\Theta} \subset \Lambda_{\downarrow}^{\Theta^{\prime}}$.

(7) Cartesian product $\Longrightarrow$ tensor product. If $\Theta$ and $\Theta^{\prime}$ are two data sets, then

$$
\Lambda_{\downarrow}^{\Theta \times \Theta^{\prime}}=\Lambda_{\downarrow}^{\Theta} \otimes \Lambda_{\downarrow}^{\Theta^{\prime}}
$$

(8) Constructible. The space $\Lambda_{\downarrow}$ can be constructed in finitely many arithmetic steps.

Notice that once an interpolating family is chosen, the actual interpolation problem reduces to solving a linear system of equations. Indeed, one would choose a linear combination of a basis generating $\Lambda_{\downarrow}$ and write a linear system for the coefficients using the values of the function we wish to interpolate on the interpolating data. The solution of the linear system will define the proper combination of the basis and hence the interpolating polynomial for the function.

It is essential that our construction of multispace ensures that the interpolation problem satisfies Properties (2) and (3) of the above theorem. To quote de Boor and Ron [13], concerning Property (2) in the above theorem, (note that $\Lambda_{\downarrow}$ is denoted as $\Pi_{\Theta}$ in the original [13])

“...If $\Theta \subset \mathbb{R}^{3}$ consists of three points, then one would choose $\Pi_{\Theta} \subset \Pi_{1}$ (as our scheme does) but if one of the three points approaches some point between the two other points, this choice has to change in the limit and hence cannot change continuously. As it turns out, our scheme is continuous at every $\Theta$ for which $\Pi_{k} \subseteq \Pi_{\Theta} \subseteq \Pi_{k+1}$, for some $k . "$

Next, we quote de Boor and Ron [13], concerning Property (3) in the above theorem.

“...If, eg, a point spirals in on another, then we cannot hope for osculation. But if, eg, one point approaches another along a straight line, then we are entitled to obtain, in the limit, a match at that point also of the directional derivative in the direction of that line."

These limitations on continuity and coalescence mean that in our construction of our multispace, we cannot be as free in our choice of generalisation of the one-dimensional pointed curves used to construct Olver's one-dimensional multispace, as might seem possible. We return to this discussion in Sect. 4.3.

Example 4.9 In the Lagrange interpolation case, assume $\Theta \subset \mathbb{R}^{p}$ is given, as before, by $(1,2)$ and $(-1,1)$, and assume $\left\{\lambda_{\theta}\right\}_{\theta \in \Theta}$ are the associated point-evaluation functionals. Thus, we have two series generating $\widehat{\Lambda}$, namely

$$
e^{x+2 y}=1+x+2 y+o(\|\mathbf{x}\|), \quad e^{-x+y}=1-x+y+o(\|\mathbf{x}\|) .
$$

A basis for the vector space generated by these two series are the series of $e^{x+2 y}=$ $1+x+2 y+o(\|\mathbf{x}\|)$ and $e^{x+2 y}-e^{-x+y}=2 x+y+o(\|\mathbf{x}\|)$, and so

$$
\Lambda_{\downarrow}=\operatorname{span}\{1,2 x+y\}
$$

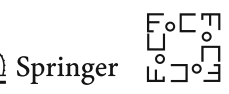


If we choose as $\Theta$ the points $(1,2)$ and $(1,-1)$, then the generators of $\widehat{\Lambda}$ are the same as before, but

$$
\Lambda_{\downarrow}=\operatorname{span}\{1, y\}
$$

as expected.

If we choose four points of the form $(1,2),(1+\epsilon, 2),(-1,1),(-1,1+\epsilon)$, then the four series generating $\widehat{\Lambda}$ are

$$
\begin{aligned}
& f_{1}=1+(1+\epsilon) x+2 y+\frac{1}{2}((1+\epsilon) x+2 y)^{2}+o\left(\|\mathbf{x}\|^{2}\right), \\
& f_{2}=1-x+(1+\epsilon) y+\frac{1}{2}(-x+(1+\epsilon) y)^{2}+o\left(\|\mathbf{x}\|^{2}\right)
\end{aligned}
$$

and

$$
\begin{aligned}
& f_{3}=1+x+2 y+\frac{1}{2}(x+2 y)^{2}+o\left(\|\mathbf{x}\|^{2}\right), \\
& f_{4}=1-x+y+\frac{1}{2}(-x+y)^{2}+o\left(\|\mathbf{x}\|^{2}\right) .
\end{aligned}
$$

Since $\left(f_{1}-f_{3}\right)_{\downarrow}=\epsilon x$ and $\left(f_{2}-f_{4}\right)_{\downarrow}=\epsilon y$, if $\epsilon \neq 0$, then $1, x$ and $y$ will be three of the four generators for $\Lambda_{\downarrow}$. A fourth will be given by

$$
\left(\frac{1}{\epsilon}\left(2\left(f_{1}-f_{3}\right)+f_{2}-f_{4}\right)-f_{3}+f_{4}\right)_{\downarrow}=(2+\epsilon) x^{2}-\frac{1-\epsilon}{2} y^{2},
$$

and hence

$$
\Lambda_{\downarrow}=\operatorname{span}\left\{1, x, y,(2+\epsilon) x^{2}-\frac{1-\epsilon}{2} y^{2}\right\}
$$

will generate the interpolating polynomials.

Example 4.10 In the Hermite interpolation case, assume we would like to instead find interpolating polynomials that coincide with a function at $(1,2)$ and $(-1,1)$, and, say, with its partial with respect to $x$ at $(1,2)$ and with respect to $y$ at $(-1,1)$. In this case, the polynomials $q_{i}$ generating the Hermite data are, at $(1,2), q_{1}(x, y)=x$, and at $(-1,1) q_{2}(x, y)=y$. One can see (see $\left.[11,12]\right)$ that $\lambda_{q_{1},(1,2)}$ is represented by the analytic function $f(\mathbf{x})=x e^{x+2 y}$, while $\lambda_{q_{2},(-1,1)}$ is defined by $g(\mathbf{x})=y e^{-x+y}$. Since $\Lambda=\operatorname{span}\left\{\lambda_{(1,2)}, \lambda_{(-1,1)}, \lambda_{q_{1},(1,2)}, \lambda_{q_{2},(-1,1)}\right\}, \widehat{\Lambda}$ has four generators, namely

$$
f_{1}=x e^{x+2 y}=x+x^{2}+2 y x+o\left(\|\mathbf{x}\|^{2}\right), \quad f_{2}=y e^{-x+y}=y-x y+y^{2}+o\left(\|\mathbf{x}\|^{2}\right)
$$


and

$$
\begin{aligned}
& f_{3}=e^{x+2 y}=1+x+2 y+\frac{1}{2}(x+2 y)^{2}+o\left(\|\mathbf{x}\|^{2}\right), \\
& f_{4}=e^{-x+y}=1-x+y+\frac{1}{2}(-x+y)^{2}+o\left(\|\mathbf{x}\|^{2}\right)
\end{aligned}
$$

Some simple and direct calculations show that $\left(f_{3}\right)_{\downarrow}=1,\left(f_{1}\right)_{\downarrow}=x,\left(f_{2}\right)_{\downarrow}=y$ and $\left(2 f_{1}+f_{2}-f_{3}+f_{4}\right)_{\downarrow}=2 x^{2}-\frac{1}{2} y^{2}$. Thus,

$$
\Lambda_{\downarrow}=\operatorname{span}\left\{1, x, y, 2 x^{2}-\frac{1}{2} y^{2}\right\} .
$$

The interpolating polynomials in the previous example converge to these as $\epsilon \rightarrow 0$.

We are now ready to define a lattice-based multispace in several variables.

\subsection{Multispaces in Several Variables}

We define two related versions of multispace, the first containing the jet bundle $J^{\ell}(M, \mathbb{R})$ and the second containing the jet bundle $J^{\ell}(U, M)$ where $U \subset \mathbb{R}^{p}$ is open.

We first recall that a point in the jet bundle $J^{r}(M, \mathbb{R})$ is represented by a triple $[x, f, U]_{r}$ where $x \in U \subset M$, the set $U$ is open, and $f: U \rightarrow \mathbb{R}$, is a $\mathcal{C}^{r}$ function. We say that the triple $[x, f, U]_{r} \sim\left[x^{\prime}, f^{\prime}, U^{\prime}\right]_{r}$ if $x=x^{\prime}$ and if, in some coordinate chart containing $x, f$ and $f^{\prime}$ have the same derivatives up to order $r,[26$, p. 60]. The equivalence class $[x, f, U]_{r}$ is known as the $r$-jet of $f$ at $x$. If $T_{r}(f)(x)$ is the order $r$ Taylor polynomial of the (sufficiently smooth) function $f$ at $x$, then $[x, f, U]_{r} \sim$ $\left[x, T_{r}(f)(x), U\right]_{r}$, so we speak of $T_{r}(f)(x)$ as being the $r$-jet of $f$ at $x$. Further, the coefficients of the $r$ th-order Taylor polynomials form local coordinates of the jet bundle $J^{r}(M, \mathbb{R})$. It is this construction that we generalise first.

To construct our multispace which both contains and generalises the jet space $J^{\ell}(M, \mathbb{R})$, we proceed as follows:

1. We first define the kinds of lattices $\Gamma$ that we will take as the models of domains for a mixed discrete-continuous jet at a point in $M$. They will be sets of points in $\mathbb{Z}^{p}$ with "directional multiplicities" or more precisely, "required contact conditions" attached. Our model lattices will come equipped with a base point. We show further that these models have the properties required for the de Boor and Ron interpolation of functions on them to be smooth, both as their image in $M$ is varied and under coalescence.

2. Next, for a model lattice $\Gamma \subset \mathbb{Z}^{p} \subset \mathbb{R}^{p}$, we let $U \subset \mathbb{R}^{p}$ be an open set, diffeomorphic to the unit disc in $\mathbb{R}^{p}$, containing $\Gamma$. Let $\phi$ be a diffeomorphic map of $U$ into $M$, and $f: \phi(U) \rightarrow \mathbb{R}$ a function. Our multijet will then be an equivalence class of quadruples $[\Gamma, \phi, f, U]$, where $[\Gamma, \phi, f, U] \sim\left[\Gamma^{\prime}, \phi^{\prime}, f^{\prime} U^{\prime}\right]$, if the base points of $\phi(\Gamma)$ and $\phi\left(\Gamma^{\prime}\right)$ agree; if $\phi(\Gamma)=\phi^{\prime}\left(\Gamma^{\prime}\right)$ as sets; if whenever $u_{J}=\phi\left(x_{J}\right)=\phi^{\prime}\left(x_{J^{\prime}}^{\prime}\right)$ the required contact conditions on $x_{J}$ and $x^{\prime} J^{\prime}$ are the

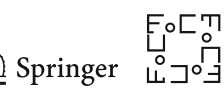



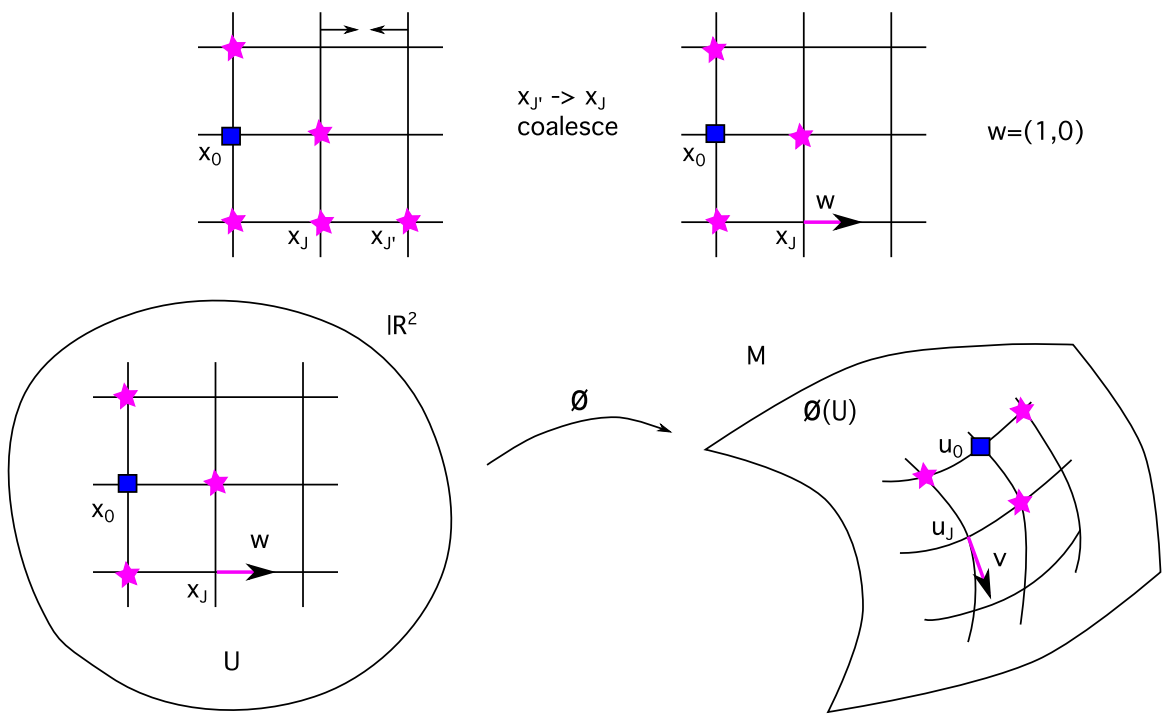

Fig. 11 After coalescence, the zeroth-order contact condition at $u_{J^{\prime}}$ is replaced by $\left.D\left(f^{\prime}\right)\right|_{u_{J}}(\mathbf{v})=$ $\left.D(f)\right|_{u_{J}}(\mathbf{v})$ where $\mathbf{v}=\left.D(\phi)\right|_{x_{J}}(\mathbf{w})$

same; and if the contact conditions induced on $\phi(\Gamma)$ by those on $\Gamma$ are all zero when evaluated on $f-f^{\prime}$, see Fig. 11 .

3. Finally, the multispace fibre over $x$ will be defined as the union of all equivalence classes of multispace jets with base point $x$. Our coordinates on the fibre are those which assign to each $[\Gamma, \phi, f, U]$, both the coefficients of the image of $\Gamma$ and the coefficients of the de Boor and Ron interpolant polynomial. In this way, we have the usual bundle topology on our multispace which relates naturally to both local coordinates on $M$ and to the coordinates on the fibre over $x$. Our multispace contains the jet bundle $J^{\ell}(M, \mathbb{R})$ for each $\ell$, as an embedded submanifold, specifically as multijets where the lattice is a single point with multiplicity $\left(\begin{array}{c}p+\ell \\ p\end{array}\right)$ (the number of derivative terms up to order $\ell$ on $p$-space), and the interpolation is given by the $\ell$ th order Taylor polynomial.

The second multispace we will define, containing $J^{\ell}(\Omega, M)$ where $\Omega$ is an open set of $\mathbb{R}^{p}$, is related to the first, by considering the function $f$, in the above construction, to be each of the coordinate functions on $M$, evaluated on the image of $U$.

\subsubsection{Basic Definitions}

As before, from now on we will assume that our lattices are connected.

Definition 4.11 We say the lattice $\Gamma$ has an $\ell$-corner distribution, or a corner distribution of length $\ell$, if it has the following inductive description:

If $p=1$, the lattice is a connected lattice with $\ell+1$ vertices. Notice that the number $\ell$ refers to the degree of the derivative one gets when all points coalesce into one point, not to the number of points in the sublattice. 
Fig. 12 Corner lattices will allow the definition of finite differences needed to approximate a Taylor polynomial

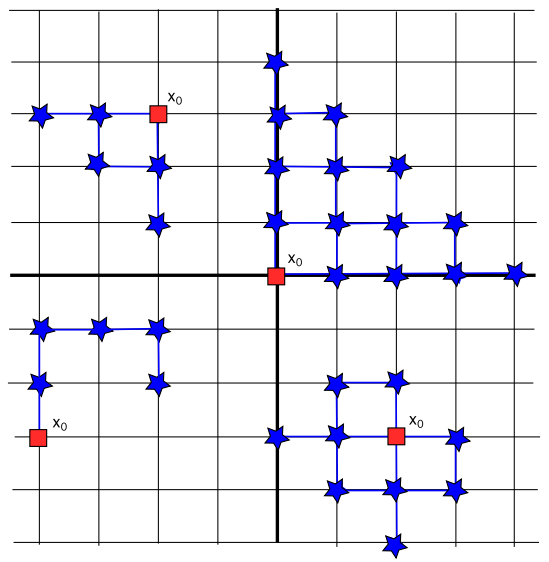

Fig. 13 A forward $p=3$ corner lattice of length $\ell=3$

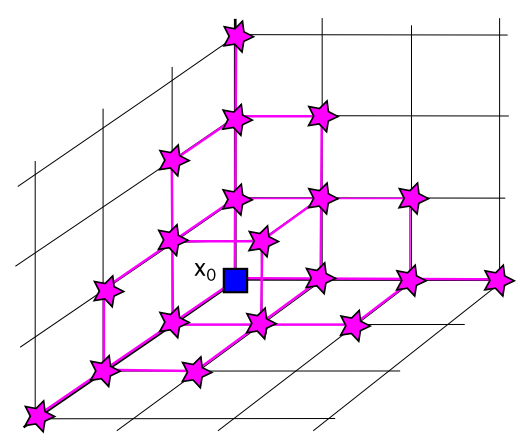

For any $p$ the lattice is a connected lattice containing $\ell+1,(p-1)$-dimensional disjoint corner lattices of lengths $0,1, \ldots, \ell$. Figure 12 shows four corner lattices for $p=2$ of lengths 4, 3, 2 and 2 (clockwise from the first quadrant). Figure 13 shows a forward $p=3$ corner distribution. We will consider corner lattices with a distinguished point $x_{0}$.

Corner distributions contain exactly enough points to define interpolating polynomials that will converge to Taylor polynomials upon coalescing. For example, if $p=2$, and $\ell=2$, and $u_{0,0}$ is the base point, a possible interpolating polynomial will have coefficients which are a linear combination of the terms

$$
1, \Delta_{x} f\left(u_{0,0}\right), \Delta_{y} f\left(u_{0,0}\right), \Delta_{x}^{2} f\left(u_{0,0}\right), \Delta_{y}^{2} f\left(u_{0,0}\right), \Delta_{y} \Delta_{x} f\left(u_{0,0}\right)
$$

where $\left.\Delta_{x}\right|_{u_{0,0}}$ is the operator $\left.\Delta_{x}\right|_{u_{0,0}}(f)=f\left(u_{1,0}\right)-f\left(u_{0,0}\right)$, and similarly with $\left.\Delta_{y}\right|_{u_{0,0}}$. To be able to uniquely determine an interpolating polynomial with those coefficients we will need to use all the vertices in a corner distribution like the one in the first quadrant of Fig. 12, with length $\ell=2$ instead of 3. Different corner lattices will produce different types of interpolating polynomials, using forward, backwards or other types of differences.

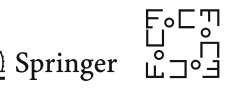


From now on we will assume that all lattices $\Gamma$ have a corner distribution of length $\ell$ with base point $x_{0}$. When several lattices are involved we will denote the base point of $\Gamma$ by $x_{\Gamma}$ and the base point of $\phi(\Gamma)$ by $u_{\Gamma}=\phi\left(x_{\Gamma}\right)$.

Lemma 4.12 Assume our data $\Theta$ is given by evaluating a function on the points of a corner lattice $\Gamma$ of length $\ell$. Then

$$
\Lambda_{\downarrow}=\Pi_{\ell}
$$

Proof Consider the monomials corresponding to polynomials of order $\ell$. That is all monomials of the form $x^{I}$ where $|I| \leq \ell$ is a multi-index $I=\left(i_{1}, \ldots, i_{p}\right)$ and $x^{I}=x_{1}^{i_{1}} \ldots x_{p}^{i_{p}}$. We want to show that these monomials are generators for $\Lambda_{\downarrow}$. Thus, we want to show that they generate $f_{\downarrow}$, where $f$ is any possible linear combination of $e^{\theta \cdot x}, \theta \in \Theta$. Therefore, it suffices to show that the coefficients of the monomials $x^{I},|I| \leq \ell$, in the Taylor expansions of $e^{\theta \cdot x}$ form an invertible matrix. Notice that we have the same number of monomials as points and hence the matrix is square.

Next, notice that the coefficients of these Taylor expansions are multiples of the monomials themselves evaluated at the point $\theta$ (since we are simply substituting $x_{i}$ by $\theta_{i} x_{i}$ in the expansion). Therefore, the matrix of coefficients is given by a multiple of the matrix with rows $\left(\theta^{I}\right), \theta \in \Theta$, where $\theta^{I}$ has the different monomials in some prescribed order. This means that if the matrix were not to be invertible, we would have a combination $\sum_{I} a_{I} \theta^{I}=0$ for $a_{I} \in \mathbb{R}$ which will be valid for all $\theta \in \Theta$. That is, the points in $\Theta$ lie in an algebraic variety of order $\ell$.

But this is not possible: Our points lie on $\ell+1$ distinct hyperplanes, and $\ell$ of them contain enough points to make the hyperplane unique (only one of them contains a single point and does not determine it). That means the polynomial must factor through the $\ell$ linear equations that define the hyperplanes and must have at least order $\ell$. But the extra single point left does not belong to any of the hyperplanes, and hence to ensure the point also lies in the variety we will need to use a polynomial of order at least $\ell+1$. Therefore, the matrix of coefficients is invertible and

$$
\Lambda_{\downarrow}=\Pi_{\ell}
$$

This lemma ensures that property (2) in Theorem 4.8 is satisfied when we use corner lattices. Next, we consider coalescence of the points in the lattice, leading to a change in the lattice, an increase in the contact order and to our Lagrange interpolation becoming a Hermite interpolation. We restrict the coalesce to be along coordinate hyperplanes in the model lattices; we call these kinds of coalescence hyperplane coalescence. See Fig. 14. Forbidden coalescences are also illustrated in Fig. 15. We note that hyperplane coalescence maintains the basepoint of the lattice, although not in general the contact condition there. Coalescence means, in effect, that we consider some lattice points to not be distinct.

Since we want the multispace to be closed under hyperplane coalescence, we consider coalesced model lattices to come equipped with certain required contact 

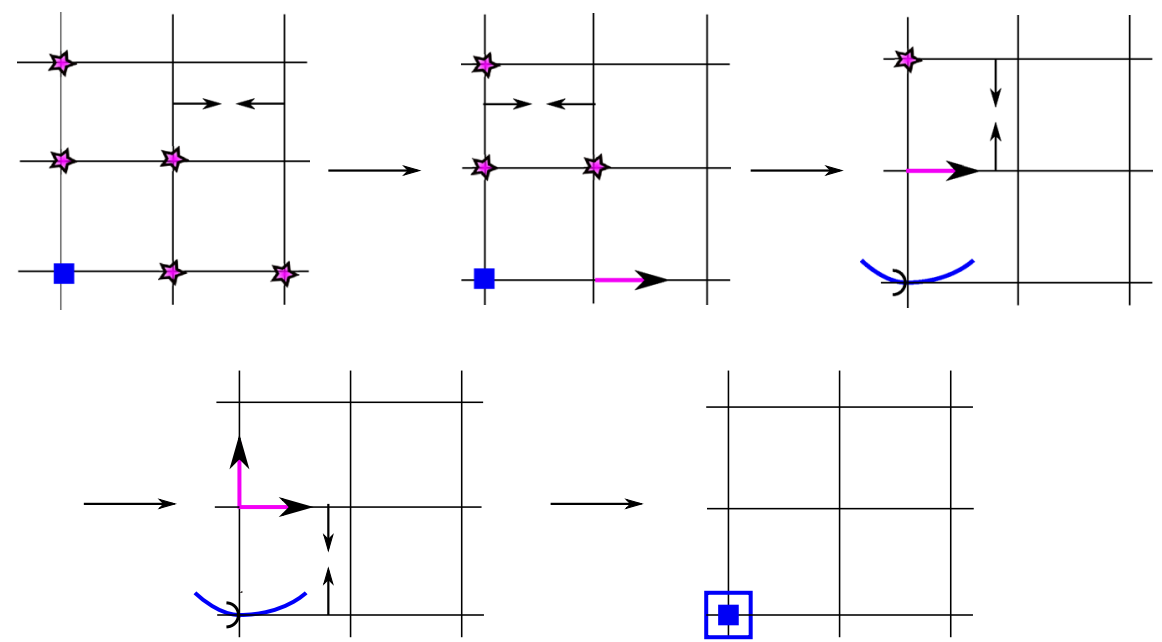

Fig. 14 Corner lattices under repeated coalescence of hyperplanes, indicated by black arrows. A red arrow indicates a zeroth- and a first-order contact condition is required at that point in the interpolation, an arc indicates a zeroth-, first- and second-order contact condition, a plane indicates all zeroth-, first- and secondorder contact conditions in the plane are required. The squared points are the base points (Color figure online)
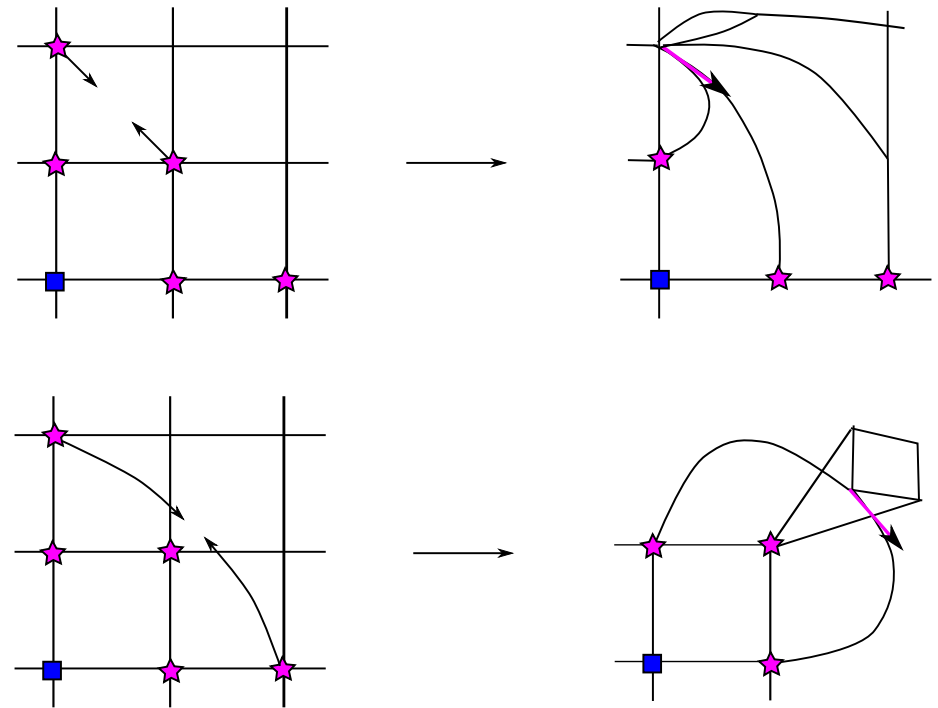

Fig. 15 Examples of forbidden coalescence. We restrict coalescence to being along coordinate hyperplanes, which maintains a coordinate structure

conditions specified at particular points of the lattice. Suppose two points, $u_{0}$ and $u_{1}=u_{0}+h \mathbf{v}$ coalesce as $h \rightarrow 0$. Then the interpolation goes from matching the values of the function $f$ at $u_{0}$ and $u_{1}$, to matching the values $f\left(u_{0}\right)$ and $\left.D(f)\right|_{u_{0}}(\mathbf{v})$. See Fig. 11. 
Repeated coalescence leads to higher-order Hermite interpolation problems. If you begin with a corner lattice and coalesce along hyperplanes repeatedly, you will arrive at a single point at which the interpolation for a function $f$ is simply the Taylor polynomial for $f$, with the order of the Taylor polynomial being the length of the corner lattice. In this way, the jet bundle $J^{\ell}(M, \mathbb{R})$ is a subset of our multispace. See Fig. 14.

From now on we will abuse the notation and denote the (coalesced) model lattice also as $\Gamma$ and the contact conditions on it by $\mathcal{C}(\Gamma)$. Under the map $\phi: U \supset \Gamma \rightarrow M$, $\phi(\Gamma)$ inherits contact conditions which we will denote by $\mathcal{C}(\phi(\Gamma))$.

The result of Lemma 4.12 remains unchanged under coalescence, as shown in the next lemma.

Lemma 4.13 Assume $\Gamma$ is a hyperplane coalesced corner lattice of length $\ell$. Then $\Lambda_{\downarrow}=\Pi_{\ell}$

Proof We can show this by induction. Assume that $\widehat{\theta}_{\hat{J}}=\theta_{J}+h e_{i}$ approaches $\theta_{J}$ along the $e_{i}$ direction, that is, as $h \rightarrow 0$, for $J \in \Gamma, \hat{J} \in \widehat{\Gamma}$ (that is for those data points in a hyperplane coalescing into another one, $\Gamma$ and $\widehat{\Gamma}$ are indexing the two hyperplanes). Except for the coalescing of a corner, we would have more than one point coalescing into one since we are using limits of hyperplanes. That is, for a given $J$, we will have more than one $\hat{J}$ limiting it. Once more we want to prove that the coefficients of the monomials $x^{I}$ in the expansion of $\exp (\theta \cdot \mathbf{x})$, for any $\theta \neq \widehat{\theta}_{\hat{J}}$ and $x_{i} \exp \left(\theta_{J} \cdot \mathbf{x}\right)$ for a given $J \in \Gamma$, and any $\hat{J} \in \widehat{\Gamma}$ related under the limit, define an invertible matrix. Let us fix $J \in \Gamma$ and let us number those limiting $\theta_{J}$ as $\theta_{j}, j=1, \ldots, p$. As in the proof of Lemma 4.12, the coefficient of $x^{I}$ in $\exp \left(\theta_{i} \cdot \mathbf{x}\right)$ is given by $\theta_{i}^{I} / I$ !, and one can directly check that the coefficient of $x^{I}$ in $x_{i} \exp \left(\theta_{j} \cdot \mathbf{x}\right)$ is given by

$$
\frac{1}{I !} \theta_{j}^{I-e_{i}} k_{i}
$$

if $I=\left(k_{1}, \ldots, k_{p}\right)$ and $I-e_{i}=\left(k_{1}, \ldots, k_{i}-1, \ldots, k_{p}\right)$, for $j \in \mathcal{J}$. Assume that the matrix formed by these coefficients is not invertible. It means that there is a polynomial of degree $\ell$ of the form

$$
\sum_{|I| \leq \ell} a_{I} \mathbf{x}^{I}
$$

that vanishes on all $\theta \in \Theta$ except for $\widehat{\theta_{j}}$. As we saw in the proof of Lemma 4.12, we would need an $\ell$-order polynomial to describe a polynomial vanishing on those points, given by the product of $\ell$ linear equations representing the $\ell$ hyperplanes (one of them will not be unique if it corresponds to a corner, since it is determined only by one point, but we can just make any choice). Now, if the lemma were not true, we would additionally need to satisfy the relation 


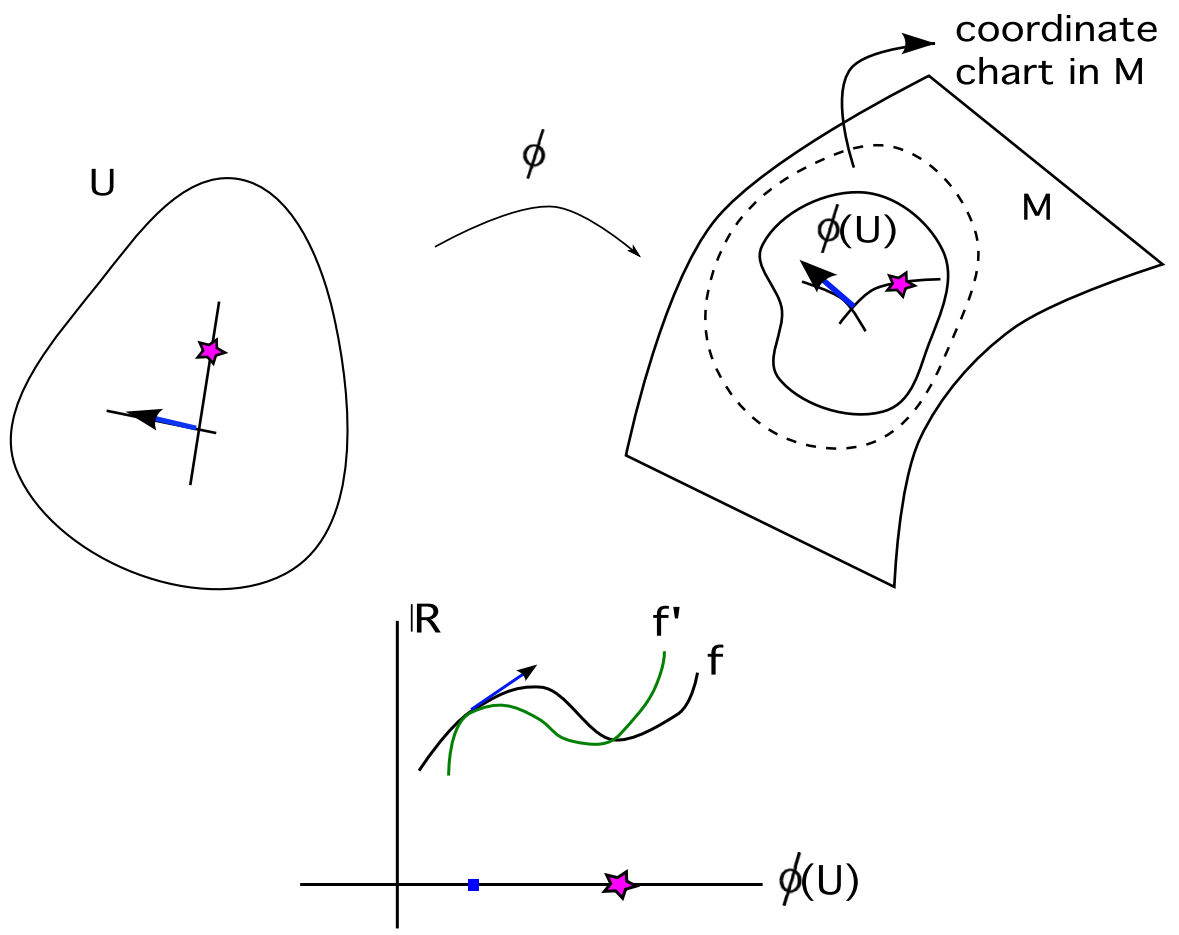

Fig. 16 The data for a multispace element. The figure shows functions $f$ and $f^{\prime}$ which agree on the contact conditions at the lattice points, $\phi(\Gamma)$. We have $[\Gamma, \phi, f, U] \sim\left[\Gamma, \phi, f^{\prime}, U\right]$

$$
\sum_{|I| \leq \ell} a_{I} \theta_{j}^{I-e_{i}} k_{i}=0
$$

where $I=\left(k_{1}, \ldots, k_{p}\right)$. This is simply the derivative of the polynomial (14) with respect to $x_{i}$. Thus, the polynomial (14) needs to have order of contact 2 at the points on the hyperplane that coalesced, Typically one would need a second-order polynomial along the hyperplane to achieve that, which would increase the degree of (14) to $\ell+1$. Higher orders of contact would result from a higher number of coalescing hyperplanes and the order of the polynomial would generically increase accordingly, proving the lemma.

The definition of elements of our multispace as equivalence classes of functions which agree on images of lattices, is illustrated in Fig. 16.

Definition 4.14 We define the multispace $(M, \mathbb{R})_{p}^{(\ell)}$ as the set of equivalence classes of quadruples $[\Gamma, \phi, f, U]$, where $(\Gamma, \mathcal{C}(\Gamma))$ is a (possibly hyperplane coalesced) corner lattice of length $\ell ; U$ is an open set of $\mathbb{R}^{p}$ for some $p \leq \operatorname{dim}(M)$, diffeomorphic to the unit disc and containing $\Gamma$; the map $\phi: U \rightarrow M$ is an embedding of $U$ into a single coordinate chart of $M$, and the map $f: \phi(U) \rightarrow \mathbb{R}$ is smooth of order $\ell$. We say two quadruples are equivalent, $[\Gamma, \phi, f, U] \sim\left[\Gamma^{\prime}, f^{\prime}, \phi^{\prime}, U^{\prime}\right]$, if the base points of $\phi(\Gamma)$, $\phi\left(\Gamma^{\prime}\right)$ agree, that is $\phi\left(x_{\Gamma}\right)=\phi^{\prime}\left(x_{\Gamma^{\prime}}\right)$, if $\phi(\Gamma)=\phi^{\prime}\left(\Gamma^{\prime}\right), \mathcal{C}(\phi(\Gamma))=\mathcal{C}\left(\phi^{\prime}\left(\Gamma^{\prime}\right)\right)$ and

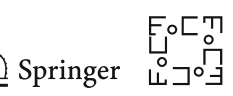


the contact conditions evaluate to zero on $f-f^{\prime}$, that is, both $f$ and $f^{\prime}$ satisfy the same contact conditions on each vertex of $\phi(\Gamma)$. (We note the contact conditions are linear.)

\subsubsection{The Main Theorem: Multispaces are Manifolds Which Contain the Jet Bundle}

Denote by $\mathcal{L}_{p}^{\ell}(M, \mathbb{R})$ the subset of $(M, \mathbb{R})_{p}^{(\ell)}$ given by non-coalesced lattices, that is a lattice with zeroth-order contact conditions at every vertex. Denote also by $J_{p}^{\ell}(M, \mathbb{R})$ the space of regular jets of maps from $p$-dimensional submanifolds of $M$ to $\mathbb{R}$. The main purpose of this section is to prove the following theorem.

Theorem 4.15 Let $M$ be a manifold of dimension $m$. Then, there exists a topology and a differential structure that makes $(M, \mathbb{R})_{p}^{(\ell)}$ into a smooth manifold of dimension $(m+1)\left(\begin{array}{c}p+\ell \\ p\end{array}\right)$, with the jet space $J_{p}^{\ell}(M, \mathbb{R})$ as a smooth submanifold and with $\mathcal{L}_{p}^{\ell}(M, \mathbb{R})$ as an open submanifold.

Before we start proving the theorem we recall that the main difficulty in defining interpolating polynomials is determining the family of polynomials with which we choose to interpolate. Once this is determined, the actual interpolating coefficients are simply given by the solution of a linear system of equations defined by the equality conditions we need to satisfy at the chosen points. Therefore, they will change smoothly insofar as the linear system (and hence the family of interpolating polynomials) changes continuously. Lemmas 4.12 and 4.13 together with Theorem 4.8 show that we are indeed in the smooth regime of the de Boor and Ron interpolation method.

Proof First of all, let us show that $(M, \mathbb{R})_{p}^{(\ell)}$ is a bundle over the manifold $M$. Let us call $x_{\Gamma}$ the base point of $\Gamma$. The fibre over $u \in M$ is the set of equivalence classes, $[\Gamma, \phi, f, U]$ where $u$ satisfies $\phi\left(x_{\Gamma}\right)=u$. Define $\pi:(M, \mathbb{R})_{p}^{(\ell)} \rightarrow M$ to be the projection map

$$
\pi([\Gamma, \phi, f, U])=\phi\left(x_{\Gamma}\right)=u .
$$

Let us first restrict to $\mathcal{L}_{p}^{\ell}(M, \mathbb{R})$. In order to have a well-defined coordinate labelling on the lattice, we need to describe an ordering on the vertices. The first point will always be the base point. Although any ordering will do, we can order them by induction: if the lattice is one-dimensional we move from smallest coordinate to largest coordinate in $\mathbb{R}$. If the lattice is two-dimensional, then we order the hyperplanes from smallest length to largest length, and then order in each hyperplane as in the one-dimensional case. In addition to its place in the ordering of the lattice points, each point in the lattice will have a coordinate in $M$. Since the image of the lattice lies in a single coordinate chart, these coordinates are consistent across the lattice, once the chart is designated. Thus the dimension of the set of embedded lattices is $m\left(\begin{array}{c}p+\ell \\ p\end{array}\right)$. This set can be considered as a submanifold of $M^{N}$, where $N=\left(\begin{array}{c}p+\ell \\ p\end{array}\right)$, with the subspace topology, and evidently has coordinates in terms of the coordinates on $M$.

If the lattices are coalesced, the coalesced vertices will have repeated coordinates, as many as the multiplicity requires. The set of coalesced lattices will have measure zero since it is the level set of some functions depending on coordinates. 
If we now consider the relevant polynomial interpolation $p_{f}$ of a (sufficiently smooth) function $f$ on the embedded image of the corner lattice, we find that it can be described by $\left(\begin{array}{c}p+\ell \\ p\end{array}\right)$ coefficients. Since, by construction, $[\Gamma, \phi, f, U] \sim\left[\Gamma, \phi, p_{f}, U\right]$, we may add these coefficients to the coordinates of the equivalence class. That these coefficients can be used as coordinates follows from knowing that we are in the regime where the de Boor and Ron interpolation is unique and depends smoothly on the data, and from the fact that the coordinates of interpolations on the coalesced lattices are well behaved limit points of those on the non-coalesced lattices.

A simple counting tells us that we have, in total, $(m+1)\left(\begin{array}{c}p+\ell \\ p\end{array}\right)$ coordinates needed to specify the equivalence class in each element of the fibre.

The bundle $(M, \mathbb{R})_{p}^{(\ell)}$ is taken to be the disjoint union of the fibres, and $\pi$ as in (15) is its projection. We may take the usual bundle topology given by the smoothness of the local trivialisations defined by the coordinates

$$
V \subset(M, \mathbb{R})_{p}^{(\ell)} \rightarrow \mathbb{R}^{m\left(\begin{array}{c}
p+\ell \\
p
\end{array}\right)} \times \mathbb{R}^{\left(\begin{array}{c}
p+\ell \\
p
\end{array}\right)}
$$

which turns the space into a manifold. The map $\pi$ is clearly smooth since the basepoint is simply the first element in the list of lattice points.

We have already shown that $J_{p}^{\ell}(M, \mathbb{R}) \subset(M, \mathbb{R})_{p}^{(\ell)}$, indeed, it is embedded as the submanifold whose first set of coordinates is the diagonal $\Delta$ in $M^{N}$, where $N=\left(\begin{array}{c}p+\ell \\ p\end{array}\right)$. We note the standard jet space is $J^{\ell}(M, \mathbb{R})=J_{m}^{\ell}(M, \mathbb{R})$.

Notice that if we perturb slightly an uncoalesced lattice $\Gamma$, the lattice will remain uncoalesced, and so the subspace of classes of the form $[\Gamma, \phi, f, U]$, with $\Gamma$ an uncoalesced corner lattice, is open.

Remark 4.16 Although we have required the image of a lattice in $M$ to be within a single coordinate chart of $M$, this restriction is perhaps not vital. The generalisation requires, firstly, keeping track of which chart as well as the coordinate given by the chart, in the lattice part of the coordinates for the multispace element. Secondly, it requires the construction of interpolations which agree on intersections of coordinate charts.

We now construct our second multispace, $(\Omega, M)_{p}^{(\ell)}$, where $\Omega \subset \mathbb{R}^{p}, p \leq \operatorname{dim} M$, which represents local approximations to embedded parametrised $p$-submanifolds in M.

Definition 4.17 We define the multispace $(\Omega, M)_{p}^{(\ell)}$ to be the set of equivalence classes of triples $[\Gamma, \phi, U]$ where $\Gamma \subset U \subset \Omega \subset \mathbb{R}^{p}$ is a (possibly coalesced) lattice of length $\ell ; U$ is an open set of $\Omega \subset \mathbb{R}^{p}$, diffeomorphic to the unit disc, where $\phi: U \rightarrow M$ is smooth of order $\ell$ and where $\phi(U)$ is contained in a single coordinate chart of $M$. We say two triples are equivalent, $[\Gamma, \phi, U] \sim\left[\Gamma^{\prime}, \phi^{\prime}, U^{\prime}\right]$ if the base points of $\Gamma, \Gamma^{\prime}$ agree; if $\phi(\Gamma)=\phi^{\prime}\left(\Gamma^{\prime}\right)$; if $\mathcal{C}(\phi(\Gamma))=\mathcal{C}\left(\phi^{\prime}\left(\Gamma^{\prime}\right)\right)$; and if $\phi(U)$ and $\phi^{\prime}\left(U^{\prime}\right)$ have the same order of contact (as submanifolds) at $\phi(\Gamma)$ as indicated by $\mathcal{C}(\phi(\Gamma))$. 
Theorem 4.18 Let $M$ be a manifold of dimension $m$. There exists a topology and a differential structure that makes $(\Omega, M)_{p}^{(\ell)}$ into a smooth manifold of dimension $2 m\left(\begin{array}{c}p+\ell \\ p\end{array}\right)$.

Proof The proof of this theorem is almost identical to that of Theorem 4.15. In local coordinates in $M$, we can write $\phi=\left(\phi_{1}, \ldots, \phi_{m}\right)$. We would then apply the process in Theorem 4.15 to produce $m\left(\begin{array}{c}p+\ell \\ p\end{array}\right)$ coordinates that determine the lattice, plus $m\left(\begin{array}{c}p+\ell \\ p\end{array}\right)$ coordinates determining the interpolating polynomial for $\phi_{k}$ with data $\Gamma$, for each $k=1, \ldots, m$. The remainder of the proof is identical to that of Theorem 4.15.

The main purpose of our multispace construction is to show that a frame on a multispace is simultaneously a frame on the jet bundle and a frame on the set of all local lattice-based discretisations. We now proceed to discuss how the group action on $M$ induces a group action on multispace. We then show that a moving frame on multispace is simultaneously a smooth and a discrete frame, with the smooth frame being the limit of the discrete, and that the discrete Maurer-Cartan invariants and their syzygies coalesce to the smooth ones.

\subsubsection{The Action of a Group on $(M, \mathbb{R})_{p}^{(\ell)}$}

Let $G$ be a group acting on $M \times \mathbb{R}$,

$$
G \times M \times \mathbb{R} \rightarrow M \times \mathbb{R}
$$

Recall the equivalence classes in $(M, \mathbb{R})_{p}^{(\ell)}$ have the form $[\Gamma, \phi, f, U]$ where $\Gamma \subset$ $U \subset \mathbb{R}^{p}, \phi: U \rightarrow M$ and $f: \phi(U) \rightarrow \mathbb{R}$. Then for each $g \in G$ there is an induced action on the map $(\phi, f): U \times \phi(U) \rightarrow M \times \mathbb{R}$ given by $(g \cdot(\phi, f))(x, z)=$ $g \cdot(\phi(x), f(z))$.

Denote the components of $g \cdot(\phi, f)$ to be $g \cdot(\phi, f)=\left((g \cdot(\phi, f))_{1},(g \cdot(\phi, f))_{2}\right) \in$ $M \times \mathbb{R}$. Then the action of $G$ on $(M, \mathbb{R})_{p}^{(\ell)}$ is given by

$$
g \cdot[\Gamma, \phi, f, U]=\left[\Gamma,(g \cdot(\phi, f))_{1},(g \cdot(\phi, f))_{2}, U\right] .
$$

We note that $(g \cdot(\phi, f))_{1}$ may be an embedding only for $g$ in a neighbourhood of the identity. In this case we would have a local group action as defined in [53]. Since the action of $G$ on $M \times \mathbb{R}$ preserves the order of contact, this action is independent of the representative of the class and is thus well defined on $(M, \mathbb{R})_{p}^{(\ell)}$.

If, in a particular application $G$ acts only on $M$, one can extend to an action on $M \times \mathbb{R}$ by taking $g \cdot(z, t)=(g \cdot z, t)$, that is the identity action on the $\mathbb{R}$ coordinate.

If $\Gamma$ is a single point so that $[\Gamma, \phi, f, U]$ is an element of $J_{p}^{\ell}(M, \mathbb{R})$, the induced action is the standard prolonged action, that is, as induced by the chain rule, while if the lattice $\Gamma$ is uncoalesced and the group does not act on the parameter $\mathbb{R}$, the action is the diagonal action on $M^{N}$ where $N=\left(\begin{array}{c}p+\ell \\ p\end{array}\right)$. 


\subsubsection{The Action of a Group on $(\Omega, M)_{p}^{(\ell)}$}

Let $G$ be a group acting on $M$

$$
G \times M \rightarrow M
$$

and let $[\Gamma, \phi, U] \in(\Omega, M)_{p}^{\ell}$ be an element of our multispace. Since $\phi: U \subset \Omega \rightarrow M$, we may define $g \cdot \phi: U \rightarrow M$ by $(g \cdot \phi)(x)=g \cdot(\phi(x))$. Define the action of $G$ on $M_{p}^{(\ell)}$ to be

$$
g \cdot[\Gamma, \phi, U]=[\Gamma, g \cdot \phi, U]
$$

Again, since the action of $G$ on $U \times M$ preserves the order of contact, this action is independent of the representative of the class and is thus well-defined on $(\Omega, M)_{p}^{(\ell)}$. Further, the action restricted to $J_{p}^{\ell}(\Omega, M) \subset(\Omega, M)_{p}^{(\ell)}$ is the standard prolonged action induced by the chain rule.

Notice that one could consider more general actions on $M \times U$, but we will omit it here to avoid further complications.

\subsubsection{Moving Frames on $(M, \mathbb{R})_{p}^{(\ell)},(\Omega, M)_{p}^{(\ell)}$}

We are now in a familiar situation: we have a smooth manifold $(M, \mathbb{R})_{p}^{(\ell)}$, or $(\Omega, M)_{p}^{(\ell)}$ and the action of a Lie group $G$ on it. Thus, we can investigate the use of the standard moving frames method developed in [17] to establish the existence of a moving frame for the multispace.

Assume the action of the group $G$ on $(M, \mathbb{R})_{p}^{(\ell)}$, or $(\Omega, M)_{p}^{(\ell)}$, is such that the existence of a local moving frame is guaranteed (see Sect. 2.1). Let us choose a point in $L \in(M, \mathbb{R})_{p}^{(\ell)}$ or $(\Omega, M)_{p}^{(\ell)}$ and let $\mathcal{S}$ be a section transverse to the orbit of $G$ through $L$. Using the standard moving frame method, we would get a local moving frame $\rho$, defined for all $\hat{L} \in(M, \mathbb{R})_{p}^{(\ell)}$, or $(\Omega, M)_{p}^{(\ell)}$ in some neighbourhood of $L$, as the group element such that $\rho \cdot \hat{L} \in \mathcal{S}$. That is, $\rho$ is an equivariant continuous map

$$
\rho: \mathcal{U} \subset(M, \mathbb{R})_{p}^{(\ell)} \rightarrow G \quad\left(\text { resp. } \rho: \mathcal{U} \subset(\Omega, M)_{p}^{(\ell)} \rightarrow G\right)
$$

where $\mathcal{U}$ is an open neighbourhood of $L$.

Remark 4.19 We note that there are results detailing conditions under which an action on a jet bundle will become free and regular for a sufficiently large prolongation, that is, by considering sufficiently high-order derivative terms [17]. A discussion of the related results for a product action, under a sufficiently large number of products, is given by Boutin [5]. We conjecture that similar results will hold for actions on multispace.

Before proving our results, we give a simple example. The first example refers to the multispace, $\left(\mathbb{R}^{2}, \mathbb{R}\right)_{1}^{(\ell)}$.

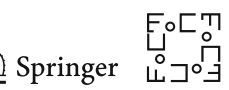


Example 4.20 We consider the two-dimensional group $G=\mathbb{R}^{+} \ltimes \mathbb{R}$ acting on $M \times$ $\mathbb{R}=\mathbb{R}^{2} \times \mathbb{R}$ as $(\lambda, \epsilon) \cdot(x, y, u(x, y))=(x, y, \lambda u+\epsilon)$. We take $\phi$ to be the identity map for simplicity. At the corner lattice $\Gamma=\left\{\left(x_{0}, y_{0}\right),\left(x_{0}+h, y_{0}\right),\left(x_{0}, y_{0}+k\right)\right\}$ with $\left(x_{0}, y_{0}\right)$ the base point, the multijet coordinates are the lattice coordinates, and the coefficients of the linear interpolant to some function $u$ on these three points. The interpolant is

$$
\begin{aligned}
p(u)(x, y)= & A+B \cdot\left(x-x_{0}\right)+C \cdot\left(y-y_{0}\right) \\
= & u\left(x_{0}, y_{0}\right)+\frac{u\left(x_{0}+h, y_{0}\right)-u\left(x_{0}, y_{0}\right)}{h}\left(x-x_{0}\right) \\
& +\frac{u\left(x_{0}, y_{0}+k\right)-u\left(x_{0}, y_{0}\right)}{k}\left(y-y_{0}\right) .
\end{aligned}
$$

Thus the coordinates coming from the interpolant are

$$
(A, B, C)=\left(u\left(x_{0}, y_{0}\right), \frac{u\left(x_{0}+h, y_{0}\right)-u\left(x_{0}, y_{0}\right)}{h}, \frac{u\left(x_{0}, y_{0}+k\right)-u\left(x_{0}, y_{0}\right)}{k}\right) .
$$

We see that the coefficients are functions of $u$ at the lattice points and so the induced group action on these coordinates is the natural action on functions. We thus have

$$
(\lambda, \epsilon) \cdot(A, B, C)=(\lambda A+\epsilon, \lambda B, \lambda C) .
$$

Under coalescence, $A \rightarrow u_{x}$ and $B \rightarrow u_{y}$, and the group action is indeed then that obtained via prolongation (i.e. the chain rule) on the jet coordinates.

Remark 4.21 (The restriction of a multispace frame to the embedded jet bundle defines a smooth frame) In the above example, we have given the normalisation equations as being for the uncoalesced lattice. The normalisation equations for the frame on the coalesced lattices and the embedded jet bundle are given implicitly by the relevant continuum limit (if this does not exist, or the result is not smooth on all multispace, then we do not have a frame on multispace). We note that normalisation equations for a frame on multispace in a domain which includes the embedded jet bundle, necessarily defines normalisation equations for a smooth frame on the embedded jet bundle, by restriction, even where their definition is given implicitly by a continuum limit. This is illustrated in Example 4.23.

Theorem 4.22 Assume $\rho$ is a local moving frame for the action of $G$ on $(M, \mathbb{R})_{p}^{(\ell)}$ (resp. $\left.(\Omega, M)_{p}^{\ell}\right)$ determined by a section transverse to an orbit of $G$. Assume that the section is also transverse to the orbit through a point $L \in J_{p}^{(\ell)}(M, \mathbb{R})$ (resp. $\left.J^{\ell}(\Omega, M)\right)$, that is, the domain of the multispace frame includes $L$.

Denote by $\rho(Q)$ the multispace frame at $Q$, and by $\rho(L)$ the smooth moving frame which is obtained by the restriction of the normalisation equations for $\rho$ to the jet space, evaluated at $L$. If $L$ and $Q$ have the same base point, then as $Q$ coalesces to $L$,

$$
\rho(Q) \rightarrow \rho(L)
$$


Proof We prove the result for $(M, \mathbb{R})_{p}^{(\ell)}$, the other case being similar. Notice that $\rho(L)$ is the standard moving frame on jet spaces obtained through a section transverse to the prolonged orbits, which is the multispace section restricted to the jet bundle. We note that $J_{p}^{\ell}(M, \mathbb{R})$ is a submanifold of $(M, \mathbb{R})_{p}^{(\ell)}$, invariant under the action of the group, so that the orbit of $G$ through a point $L \in J_{p}^{\ell}(M, \mathbb{R})$ is equal to the prolonged orbit.

The proof is now immediate from the fact that the moving frame on $(M, \mathbb{R})_{p}^{(\ell)}$ is a smooth map.

In Appendix, we will show a different convergence theorem for families of discrete frames.

Example 4.23 Continuing with our previous Example 4.20, a moving frame $\rho$ is defined by $(\lambda, \epsilon) \cdot(A, B, C)=\left(0,1,{ }^{*}\right)$ where ${ }^{*}$ will be the invariant, $\left.(\lambda, \epsilon) \cdot C\right|_{\rho}$. This yields $\left.(\lambda, \epsilon)\right|_{\rho}=\left(\frac{h}{u\left(x_{0}+h, y_{0}\right)-u\left(x_{0}, y_{0}\right)},-\frac{h u\left(x_{0}, y_{0}\right)}{u\left(x_{0}+h, y_{0}\right)-u\left(x_{0}, y_{0}\right)}\right)$ or in the standard matrix representation of this group,

$$
\rho=\left(\begin{array}{cc}
\frac{h}{u\left(x_{0}+h, y_{0}\right)-u\left(x_{0}, y_{0}\right)} & -\frac{h u\left(x_{0}, y_{0}\right)}{u\left(x_{0}+h, y_{0}\right)-u\left(x_{0}, y_{0}\right)} \\
0 & 1
\end{array}\right) .
$$

We saw that as $h \rightarrow 0$, we obtain the correct induced group action on $u_{x}$. We now see further that the limiting frame

$$
\rho\left(x, u, u_{x}\right)=\left(\begin{array}{cc}
\frac{1}{u_{x}} & -\frac{u}{u_{x}} \\
0 & 1
\end{array}\right)
$$

is obtained both from the limit of the normalisation equations, $(\lambda, \epsilon) \cdot\left(u_{x}, u\right)=(1,0)$ as well as being the limit of the frame itself. We therefore have a frame on multispace, so that both the discrete and the smooth cases are handled by the one calculation of the frame on multispace.

\subsection{The Continuous Limit of Invariants and Syzygies}

We return to our discussion of discrete moving frames in the previous section, in which we have a lattice variety $\mathcal{L}_{N}$ embedded in some manifold, and a discrete frame is a map $\rho: \mathcal{L}_{N} \rightarrow G^{N}$ where $N$ is the number of points in the lattice. Suppose now that adjacent vertices in the lattice variety begin to coalesce. Under what conditions will the discrete frame converge to a smooth frame, $\bar{\rho}$ ? Furthermore, under what kinds of conditions will we have

$$
\rho_{n+1} \rho_{n}^{-1} \sim \bar{\rho}(z+\epsilon v) \bar{\rho}(z)^{-1} \sim \exp \left(\epsilon(\mathcal{D} \bar{\rho}) \bar{\rho}^{-1}\right)
$$

where $\mathcal{D}$ is an invariant differential operator? And when will the discrete local syzygies converge to the local differential syzygies?

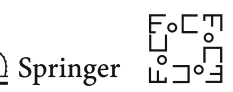


Example 4.24 Let us go back to our running Example 4.20. Setting $\rho_{i}=\rho\left(x_{i}\right.$, $\left.y_{i}, u\left(x_{i}, y_{i}\right), u\left(x_{i}+h_{i}, y_{i}\right), u\left(x_{i}, y_{i}+k_{i}\right)\right)$ and calculating $\rho_{1} \rho_{0}^{-1}$, we have, using Eq. (16),

$$
\rho_{1} \rho_{0}^{-1}=\left(\begin{array}{c}
\frac{h_{1}\left(u\left(x_{0}+h_{0}, y_{0}\right)-u\left(x_{0}, y_{0}\right)\right.}{h_{0}\left(u\left(x_{1}+h_{1}, y_{1}\right)-u\left(x_{1}, y_{1}\right)\right)} \\
0
\end{array} \frac{h_{1}\left(u\left(x_{0}, y_{0}\right)-u\left(x_{1}, y_{1}\right)\right.}{u\left(x_{1}+h_{1}, y_{1}\right)-u\left(x_{1}, y_{1}\right)}\right) .
$$

If we now set $\left(x_{1}, y_{1}\right)=\left(x_{0}+\bar{h}, y_{0}\right)$ and $h_{0}=h_{1}=h$ (say), so that the second lattice is the shift of the first, by $(\bar{h}, 0)$, then

$$
\begin{aligned}
& \left.\frac{\mathrm{d}}{\mathrm{d} \bar{h}}\right|_{\bar{h}=0} \rho_{1} \rho_{0}^{-1} \\
& =\left(\begin{array}{c}
-\frac{u_{x}\left(x_{0}+h, y_{0}\right)-u_{x}\left(x_{0}, y_{0}\right)}{u\left(x_{0}+h, y_{0}\right)-u\left(x_{0}, y_{0}\right)}-\frac{h u_{x}\left(x_{0}, y_{0}\right)}{u\left(x_{0}+h, y_{0}\right)-u\left(x_{0}, y_{0}\right)} \\
0
\end{array}\right) \\
& \rightarrow_{h \rightarrow 0}\left(\begin{array}{cc}
-\left.\frac{u_{x x}}{u_{x}}\right|_{\left(x_{0}, y_{0}\right)}-1 \\
0 & 0
\end{array}\right) \\
& =\left.\rho_{x} \rho^{-1}\right|_{J^{2}(M)} \text {. }
\end{aligned}
$$

Alternatively, using the method we will apply in Example 4.29, we have setting $u(x+$ $\bar{h}, y)=u(x, y)+\bar{h} u_{x}(x, y)+\mathcal{O}\left(\bar{h}^{2}\right)$, that

$$
\begin{aligned}
& \rho_{1} \rho_{0}^{-1} \\
& =\left(\begin{array}{c}
1-\frac{u_{x}\left(x_{0}+h, y_{0}\right)-u_{x}\left(x_{0}, y_{0}\right)}{u\left(x_{0}+h, y_{0}\right)-u\left(x_{0}, y_{0}\right)} \bar{h}+\mathcal{O}\left(\bar{h}^{2}\right) \frac{h u_{x}\left(x_{0}, y_{0}\right)}{u\left(x_{0}+h, y_{0}\right)-u\left(x_{0}, y_{0}\right)} \bar{h}+\mathcal{O}\left(\bar{h}^{2}\right) \\
0
\end{array}\right) \\
& \sim \exp \left(\bar{h}\left(\begin{array}{c}
-\frac{u_{x}\left(x_{0}+h, y_{0}\right)-u_{x}\left(x_{0}, y_{0}\right)}{u\left(x_{0}+h, y_{0}\right)-u\left(x_{0}, y_{0}\right)}-\frac{h u_{x}\left(x_{0}, y_{0}\right)}{u\left(x_{0}+h, y_{0}\right)-u\left(x_{0}, y_{0}\right)} \\
0
\end{array}\right)\right) \\
& \sim \exp \left(\bar{h} \rho_{x} \rho^{-1}\right)
\end{aligned}
$$

as above. We note that $\partial / \partial x$ is an invariant operator since the independent variables are invariant under the action, so that $\rho_{x} \rho^{-1}$ is invariant. Cases where the independent variables participate in the action require more care, as we indicate below.

Our first theorem concerns the convergence of the discrete Maurer-Cartan invariants to the smooth Maurer-Cartan invariants of the smooth frame.

We consider the case where the discrete frame $\left(\rho_{R}\right)$ on the lattice variety $\mathcal{L}_{N} \subset M$ can be viewed as a multispace frame $\rho$, with

$$
\rho_{J}=\rho\left(\left[\Gamma_{J}, \phi, U\right]\right), \quad u_{J}=\phi\left(x_{J}\right),
$$

where $x_{J}$ is the basepoint of $\Gamma_{J}$. 
Assume the point $\mathcal{T}_{i} x_{J}$ is also part of $\Gamma_{J}$ so that $\mathcal{T}_{i} u_{J}=\phi\left(\mathcal{T}_{i} x_{J}\right)$. If we have a path from $\mathcal{T}_{i}\left(u_{J}\right)$ to $u_{J}$ in the multispace indicating their coalescence, we can use the multispace frame to differentiate $\mathcal{T}_{i}\left(\rho_{J}\right) \rho_{J}^{-1}$ with respect to the path parameter at $u_{J}$. We state and prove the next theorem for a multispace of the form $\mathcal{M}=(\Omega, M)_{p}^{\ell}$, and then we discuss the other case in Remark 4.26.

Theorem 4.25 Let a multispace $\mathcal{M}$ with the embedded jet bundle $\mathcal{J}$ be given. Let a path in $\mathcal{M}$ be given, $\epsilon \mapsto[\Gamma(\epsilon), \phi, U]$ for $0 \leq \epsilon \leq 1$. Let $u(\epsilon)$ be the base point of $\phi(\Gamma(\epsilon))$. Assume that both paths and the coalescence $\phi(\Gamma(\epsilon)) \rightarrow \phi\left(\Gamma_{0}\right)$ lie in the domain of a multispace frame $\rho$. Set $\rho(\epsilon)=\rho([\Gamma(\epsilon), \phi, U])$, that is, $\rho$ evaluated at the point $[\Gamma(\epsilon), \phi, U]$. If $\mathbf{v}=\mathrm{d} /\left.\mathrm{d} \epsilon\right|_{\epsilon=0} u(\epsilon)$, then

$$
\lim _{\Gamma(0) \rightarrow \Gamma_{0}}\left[\left.\frac{d}{d \epsilon}\right|_{\epsilon=0} \rho(\epsilon) \rho^{-1}(0)\right]=(D(\mathbf{v}) \rho) \rho^{-1}
$$

where $D(\mathbf{v})=\sum v_{i} \partial / \partial x_{i}$ is the directional derivative.

The theorem follows from standard results concerning smooth functions on manifolds.

Remark 4.26 For a multispace of the form $\mathcal{M}=(M, \mathbb{R})_{p}^{(\ell)}$, it is possible that the independent variables participate in the group action, and then $(D(\mathbf{v}) \rho) \rho^{-1}$ may not be invariant. We recall that a smooth frame on a jet bundle yields a canonical, maximal set of invariant differential operators. Indeed, on a manifold with coordinates $\mathbf{u}$, if $g \cdot \mathbf{u}=\widetilde{\mathbf{u}}$, then we define

$$
\mathcal{D}_{i}=\left.\frac{\partial}{\partial \widetilde{u}_{i}}\right|_{g=\rho}=\left.\left(\frac{\partial \widetilde{\mathbf{u}}}{\partial \mathbf{u}}\right)_{i j}^{-T}\right|_{g=\rho} \frac{\partial}{\partial u_{j}}
$$

Rewriting the partial derivatives in (20) in terms of the invariantised derivatives, by inverting Eq. (21), yields an expression from which the right hand side of Eq. (18) may be obtained, provided we are careful about the curve $\epsilon \rightarrow u(\epsilon)$ used in Theorem 4.25 to obtain $\mathbf{v}$.

Consider the example of a scaling action on a single independent variable, so $M$ is the positive real line, and $G$ is the group of positive real numbers under standard multiplication. Suppose $f: M \rightarrow R$ is invariant under the group action. Let the frame be given by $\rho([\Gamma, \phi, f, U])=1 / u$ where $u$ is the image of the basepoint of $\Gamma$. Then the single invariant operator is $u \partial / \partial u$. If we take our path of coalescence to be $u(\epsilon)=u+\epsilon$, then $D(\mathbf{v})$ in the statement of the Theorem will not be invariant. However, if we take $u(\epsilon)=(1+\epsilon) u$, then it will be.

Suppose that $D(\mathbf{v})=\sum_{i} v_{i} \partial / \partial u_{i}$. Inverting Eq. (21) yields expressions of the form $\partial / \partial u_{i}=\sum_{k} A_{i k} \mathcal{D}_{k}$, so that

$$
D(\mathbf{v})=\sum_{i} v_{i} \sum_{k} A_{i k} \mathcal{D}_{k}=\sum_{k}\left(\sum_{i} v_{i} A_{i k}\right) \mathcal{D}_{k}=\sum_{k} \bar{v}_{k} \mathcal{D}_{k}=\mathcal{D}(\overline{\mathbf{v}})
$$

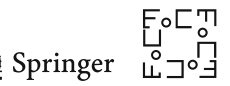


where the last equality defines the vector $\overline{\mathbf{v}}$. To ensure that $\mathcal{D}(\overline{\mathbf{v}})$ is an invariant directional derivative, we must have that the components of $\overline{\mathbf{v}}$ are either constants or more generally, invariants.

We note that for the operators $\mathcal{D}_{i}$ defined in $(21)$, that $\mathcal{D}_{i} \rho \rho^{-1}$ can be calculated using only the equations for the transverse section that determines the frame and the infinitesimal action, see [38] for details.

Example 4.27 (Special Euclidean group action on curves in the plane) The group is $\mathrm{SO}(2) \ltimes \mathbb{R}^{2}$ with the standard linear action of translation and rotation of curves on the plane, specifically,

$$
\left(\begin{array}{l}
x(t) \\
y(t)
\end{array}\right) \mapsto\left(\begin{array}{l}
\tilde{x}(t) \\
\tilde{y}(t)
\end{array}\right)=\left(\begin{array}{cc}
\cos \theta & \sin \theta \\
-\sin \theta & \cos \theta
\end{array}\right)\left(\begin{array}{l}
x(t)-a \\
u(t)-b
\end{array}\right)
$$

If one takes the standard matrix representation of $\mathrm{SO}(2) \ltimes \mathbb{R}^{2}$, so that the action involves the inverse of the group element, then the equivariance of the frame will be $\rho(g \cdot z)=g \rho(z)$.

The multispace frame calculation is as follows. We take an order 2 interpolation as we wish to achieve a multispace approximation of the curvature. If we interpolate the curve $(x(t), u(t))$ at $\Gamma=\left\{t_{0}, t_{0}+h_{1}, t_{0}+h_{2}\right\}$ with base point $t_{0}$, we get

$$
\begin{aligned}
p(x(t))= & A(x)+B(x) \cdot\left(t-t_{0}\right)+\frac{1}{2} C(x) \cdot\left(t-t_{0}\right)^{2} \\
= & x\left(t_{0}\right)+\frac{\left(h_{1}^{2}-h_{2}^{2}\right) x\left(t_{0}\right)+h_{2}^{2} x\left(t_{0}+h_{1}\right)-h_{1}^{2} x\left(t_{0}+h_{2}\right)}{h_{1} h_{2}\left(h_{2}-h_{1}\right)}\left(t-t_{0}\right) \\
& +\frac{\left(h_{2}-h_{1}\right) x\left(t_{0}\right)-h_{2} x\left(t_{0}+h_{1}\right)+h_{1} x\left(t_{0}+h_{2}\right)}{h_{1} h_{2}\left(h_{2}-h_{1}\right)}\left(t-t_{0}\right)^{2},
\end{aligned}
$$

and similarly for $p(u(t))=A(u)+B(u) .\left(t-t_{0}\right)+\frac{1}{2} C(u) \cdot\left(t-t_{0}\right)^{2}$. The induced action on the coefficients is that induced on $(x(t), u(t))$ so for example

$$
\begin{aligned}
g \cdot B(u)= & \frac{\left(h_{1}^{2}-h_{2}^{2}\right) g \cdot u\left(t_{0}\right)+h_{2}^{2} g \cdot u\left(t_{0}+h_{1}\right)-h_{1}^{2} g \cdot u\left(t_{0}+h_{2}\right)}{h_{1} h_{2}\left(h_{2}-h_{1}\right)} \\
= & \frac{1}{h_{1} h_{2}\left(h_{2}-h_{1}\right)}\left[\left(h_{1}^{2}-h_{2}^{2}\right)\left(\cos \theta\left(x\left(t_{0}\right)-a\right)+\sin \theta\left(u\left(t_{0}\right)-b\right)\right)\right. \\
& \left.\quad+h_{2}^{2}\left(\cos \theta\left(x\left(t_{0}+h_{1}\right)-a\right)+\sin \theta\left(u\left(t_{0}+h_{1}\right)-b\right)\right)\right) \\
& \left.-h_{1}^{2}\left(\cos \theta\left(x\left(t_{0}+h_{2}\right)-a\right)+\sin \theta\left(u\left(t_{0}+h_{2}\right)-b\right)\right)\right] .
\end{aligned}
$$

The normalisation equations $g \cdot A(x)=0, g \cdot A(u)=0$ and $g \cdot B(u)=0$ yield the frame at $\Gamma$ to be

$a=x\left(t_{0}\right), \quad b=u\left(t_{0}\right), \quad \tan \theta=\frac{\left(h_{1}^{2}-h_{2}^{2}\right) u\left(t_{0}\right)+h_{2}^{2} u\left(t_{0}+h_{1}\right)-h_{1}^{2} u\left(t_{0}+h_{2}\right)}{\left(h_{1}^{2}-h_{2}^{2}\right) x\left(t_{0}\right)+h_{2}^{2} x\left(t_{0}+h_{1}\right)-h_{1}^{2} x\left(t_{0}+h_{2}\right)}$. 
In the limit as $h_{2} \rightarrow h_{1}$, we have

$$
\tan \theta \rightarrow \frac{u_{t}\left(t_{0}+h_{1}\right)-2 u\left(t_{0}+h_{1}\right)+2 u\left(t_{0}\right)}{x_{t}\left(t_{0}+h_{1}\right)-2 x\left(t_{0}+h_{1}\right)+2 x\left(t_{0}\right)}
$$

and then finally as $h_{1} \rightarrow 0$, we have $\tan \theta \rightarrow u_{t} / x_{t}$ as expected, indeed, yielding the smooth frame as determined by the limit of the normalisation equations.

If we take the standard matrix representation of $\mathrm{SO}(2) \ltimes \mathbb{R}^{2}$ to represent the frame, with the equivariance as above, then the invariant Maurer-Cartan matrix will be $\rho\left(\left[\Gamma^{\prime}, u, \phi, U\right]\right)^{-1} \rho([\Gamma, u, \phi, U])$, and the components of this yield the discrete multispace Maurer-Cartan invariants for this frame. Further, $\rho \cdot B(x) \rightarrow\left(x_{t}^{2}+u_{t}^{2}\right)^{1 / 2}$ so that we may treat $\rho \cdot B(x)$ as the multispace approximation to the infinitesimal arc-length.

In the above example, we used an invariant parameter $t$ to describe the curve $(x(t), u(t))$. If instead we parametrise the curve as $(x, u(x))$, so that the parameter participates in the group action and the operator $\partial / \partial x$ is not invariant, then greater care is required. For example, the group action on the interpolation curve, written as $p(u)(x)=A(x, u)+B(x, u)\left(x-x_{0}\right)+C(x, u)\left(x-x_{0}\right)^{2}$, looks like $g \cdot p(u)(x)=A(\widetilde{x}, \widetilde{u})+B(\widetilde{x}, \widetilde{u})\left(x-\tilde{x_{0}}\right)+C(\widetilde{x}, \widetilde{u})\left(x-\widetilde{x_{0}}\right)^{2}$. Solving for the multispace frame in this case seems nontrivial. Such examples will be examined elsewhere.

Now assume we have four lattice points, $u_{J}, \mathcal{T}_{1} u_{J}, \mathcal{T}_{2} u_{J}$ and $\mathcal{T}_{2} \mathcal{T}_{1} u_{J}=\mathcal{T}_{1} \mathcal{T}_{2} u_{J}$, and that we have two paths connecting $u_{J}$ with $\mathcal{T}_{2} \mathcal{T}_{1} u_{J}=\mathcal{T}_{1} \mathcal{T}_{2} u_{J}$ via each of the $\mathcal{T}_{1} u_{J}$ and $\mathcal{T}_{2} u_{J}$ respectively. If we can associate the discrete frame with a multispace frame and differentiate the local syzygy associated with the discrete frame with respect to the path parameters at $u_{J}$, we obtain the differential syzygy associated with the multispace frame at $u_{J}$. Indeed, let a discrete frame $\left(\rho_{J}\right)$ at the points $u_{J}, \mathcal{T}_{1} u_{J}, \mathcal{T}_{2} u_{J}$ and $\mathcal{T}_{1} \mathcal{T}_{2} u_{J}$ be associated with the multispace frame $\rho$ so that $\rho_{J}=\rho\left(z_{J}\right)$ for some $z_{J}=\left[\Gamma_{J}, \phi, U\right]$ in the relevant multispace $\mathcal{M}, u_{J}=\phi\left(x_{J}\right)$. Then, the local syzygy is

$$
\mathcal{T}_{1}\left(K_{2}\right) K_{1}=\mathcal{T}_{2}\left(K_{1}\right) K_{2}, \quad K_{1}=\rho\left(\mathcal{T}_{1} z_{J}\right) \rho\left(z_{J}\right)^{-1}, \quad K_{2}=\rho\left(\mathcal{T}_{2} z_{J}\right) \rho\left(z_{J}\right)^{-1},
$$

where $\mathcal{T}_{i} z_{J}=\left[\mathcal{T}_{i} \Gamma_{J}, \phi, U\right]$ and $\mathcal{T}_{i} \Gamma_{J}$ is the shift of $\Gamma_{J}$ by $\mathcal{T}_{i}$, with base point $\mathcal{T}_{i} x_{J}$ and $\mathcal{T}_{i} u_{J}=\phi\left(\mathcal{T}_{i} x_{J}\right)=\phi\left(x_{J+e_{i}}\right)$. Here we are assuming that the shifts of $\Gamma_{J}$ remain within $U$. Now let $z_{J}^{i}\left(\epsilon_{i}\right)$ be paths in the multispace lying within $U$, with $z_{J}^{i}(0)=z_{J}$, $z_{J}^{i}(1)=\mathcal{T}_{i} z_{J}, z_{J}^{i}\left(\epsilon_{i}\right)=\left[\Gamma_{J}^{i}\left(\epsilon_{i}\right), \phi, U\right]$ and $u_{J}^{i}\left(\epsilon_{i}\right)=\phi\left(x_{J}^{i}\left(\epsilon_{i}\right)\right), x_{J}^{i}\left(\epsilon_{i}\right)$ the base point of $\Gamma_{J}^{i}\left(\epsilon_{i}\right)$. We denote

$$
\mathbf{v}=\left.\frac{\mathrm{d}}{\mathrm{d} \epsilon_{1}}\right|_{\epsilon_{1}=0} u_{J}^{1}\left(\epsilon_{1}\right), \quad \mathbf{w}=\left.\frac{\mathrm{d}}{\mathrm{d} \epsilon_{2}}\right|_{\epsilon_{2}=0} u_{J}^{2}\left(\epsilon_{2}\right),
$$

and

$$
\left.\left.K_{\mathbf{v}}=\left.\frac{\mathrm{d}}{\mathrm{d} \epsilon_{1}}\right|_{\epsilon_{1}=0} \rho\left(z_{J}^{1}\left(\epsilon_{1}\right)\right)\right) \rho\left(z_{J}\right)^{-1}, \quad K_{\mathbf{w}}=\left.\frac{\mathrm{d}}{\mathrm{d} \epsilon_{2}}\right|_{\epsilon_{2}=0} \rho\left(z_{J}^{2}\left(\epsilon_{2}\right)\right)\right) \rho\left(z_{J}\right)^{-1} .
$$

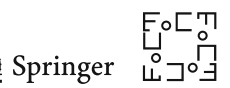


We assume that the parametrisation of these paths with respect to $\epsilon$ yield invariant differential operators in the case where the independent variables participate in the group action, see Remark 4.26.

Theorem 4.28 After differentiating twice, once each with respect to the path parameters $\epsilon_{1}$ and $\epsilon_{2}$ and coalescencing the lattice of the multispace point $z_{J}$ to its associated jet point, and under the conditions just stated, the local syzygy, Eq. (22), becomes the continuous basic syzygy associated to $\rho\left(z_{J}\right)$

$$
\mathcal{D}(\mathbf{w}) K_{\mathbf{v}}=\mathcal{D}(\mathbf{v}) K_{\mathbf{w}}+([\mathcal{D}(\mathbf{w}), \mathcal{D}(\mathbf{v})] \rho) \rho^{-1}+\left[K_{\mathbf{v}}, K_{\mathbf{w}}\right] .
$$

Proof The core of the proof is standard. We assume a matrix representation of the frame, and note that Taylor's Theorem is valid.

To ease the notation, we set $\epsilon_{1}=h$ and $\epsilon_{2}=k$, and simplify to where $\mathbf{v}=$ $\mathrm{d} /\left.\mathrm{d} h\right|_{h=0} \phi\left(x_{0}+h \mathbf{e}_{1}\right)$ and $\mathbf{w}=\mathrm{d} /\left.\mathrm{d} k\right|_{k=0} \phi\left(x_{0}+k \mathbf{e}_{2}\right)$ in local coordinates, so that we evaluate our frame at the multispace elements with lattice basepoints at $u_{J}=\phi\left(x_{0}\right)$, $\mathcal{T}_{1} u_{J}=u_{J, 1}=\phi\left(x_{0}+h \mathbf{e}_{1}\right), \mathcal{T}_{2} u_{J}=u_{J, 2}=\phi\left(x_{0}+k \mathbf{e}_{2}\right)$ and $\mathcal{T}_{2} \mathcal{T}_{1} u_{J}=u_{J, 1,2}=$ $u_{J, 2,1}=\phi\left(x_{0}+h \mathbf{e}_{1}+k \mathbf{e}_{2}\right)$. We denote the partial derivative operators $\partial / \partial \epsilon_{i}$ as $\partial_{i}$.

Denoting $\mathcal{T}_{i} \rho_{J}=\rho_{J, i}$ we have

$$
K_{J, 1}=\rho_{J, 1} \rho_{J}^{-1}, \quad K_{J, 2}=\rho_{J, 2} \rho_{J}^{-1},
$$

and for sufficiently small $h$ and $k$, there will exist, dropping the index $J$ for clarity, matrices $X_{1}$ and $X_{2}$ in the Lie algebra of $G$ such that

$$
K_{1}=\exp \left(h X_{1}+\mathcal{O}\left(h^{2}\right)\right), \quad K_{2}=\exp \left(k X_{2}+\mathcal{O}\left(k^{2}\right)\right) .
$$

We have

$$
\partial_{1} \rho \cdot \rho^{-1}=X_{1}, \quad \partial_{2} \rho \cdot \rho^{-1}=X_{2}
$$

and

$$
\mathcal{T}_{2} X_{1}=X_{1}+k \partial_{2} X_{1}+\mathcal{O}\left(k^{2}\right), \quad \mathcal{T}_{1} X_{2}=X_{2}+h \partial_{1} X_{2}+\mathcal{O}\left(h^{2}\right)
$$

Then

$$
\begin{aligned}
\mathcal{T}_{2} K_{1} & =\exp \left(h \mathcal{T}_{2} X_{1}+\mathcal{O}\left(h^{2}\right)\right) \\
& =\exp \left(h X_{1}+h k \partial_{2} X_{1}+\mathcal{O}\left(k^{2}, h^{2}\right)\right) \\
\mathcal{T}_{1} K_{2} & =\exp \left(k \mathcal{T}_{1} X_{2}+\mathcal{O}\left(k^{2}\right)\right) \\
& =\exp \left(k X_{2}+h k \partial_{1} X_{2}+\mathcal{O}\left(k^{2}, h^{2}\right)\right) .
\end{aligned}
$$

Applying the Baker-Campbell-Hausdorff formula, [59],

$$
\log (\exp (X) \exp (Y))=X+Y+\frac{1}{2}[X, Y]+\text { higher order brackets, }
$$


we have

$$
\begin{aligned}
\left.\log \left(\mathcal{T}_{2} K_{1} \cdot K_{2}\right)\right) & =h X_{1}+h k \partial_{2} X_{1}+k X_{2}+\frac{1}{2}\left[h X_{1}+h k \partial_{2} X_{1}, k X_{2}\right]+\mathcal{O}\left(k^{2}, h^{2}\right) \\
& =h X_{1}+k X_{2}+h k\left(\partial_{2} X_{1}+\frac{1}{2}\left[X_{1}, X_{2}\right]\right)+\mathcal{O}\left(k^{2}, h^{2}\right), \\
\left.\log \left(\mathcal{T}_{1} K_{2} \cdot K_{1}\right)\right) & =k X_{2}+h k \partial_{1} X_{2}+h X_{1}+\frac{1}{2}\left[k X_{2}+h k \partial_{1} X_{2}, h X_{1}\right]+\mathcal{O}\left(k^{2}, h^{2}\right) \\
& =h X_{1}+k X_{2}+h k\left(\partial_{1} X_{2}+\frac{1}{2}\left[X_{2}, X_{1}\right]\right)+\mathcal{O}\left(k^{2}, h^{2}\right) .
\end{aligned}
$$

Equating the two formulae by imposing the local syzygy, differentiating with respect to both $h$ and $k$ and then sending $h, k \rightarrow 0$, yields, after a slight rearrangement,

$$
\partial_{2} X_{1}-\partial_{1} X_{2}=\left[X_{2}, X_{1}\right] \text {. }
$$

Finally, we need to rewrite Eq. (25) in terms of the invariant differential operators. The formula given in Eq. (21) shows that the partial derivatives $\partial_{i}$ can be written as a linear sum of the invariant operators with invariant coefficients. We must then back substitute for the $\partial_{i}$, including rewriting the $X_{i}=\partial_{i} \rho \cdot \rho^{-1}$ in terms of the $\mathcal{D}_{j} \rho \cdot \rho^{-1}$. The final result yields the extra terms in the case that the invariant operators do not commute.

Example 4.29 We conclude the running Example 4.20. We set the points $\left(x_{i}, y_{i}\right)$, $i=1,2,3$ to be $\left(x_{1}, y_{1}\right)=\left(x_{0}+\bar{h}, y_{0}\right),\left(x_{2}, y_{2}\right)=\left(x_{0}, y_{0}+\bar{k}\right)$ and $\left(x_{3}, y_{3}\right)=$ $\left(x_{0}+\bar{h}, y_{0}+\bar{k}\right)$. We then calculate the four matrices, $K_{10}=\rho_{1} \rho_{0}^{-1}=\exp \left(X_{10}\right)$, $K_{31}=\rho_{3} \rho_{1}^{-1}=\exp \left(X_{31}\right), K_{20}=\rho_{2} \rho_{0}^{-1}=\exp \left(X_{20}\right)$ and $K_{32}=\rho_{3} \rho_{2}^{-1}=$ $\exp \left(X_{32}\right)$. Direct calculation gives, setting $\Delta_{1} F=F\left(x_{0}+h, y_{0}\right)-F\left(x_{0}, y_{0}\right)$ for a function $F \in\left\{u, u_{x}, u_{y}, u_{x y}\right\}$ in the formulae to ease the notation,

$$
\begin{aligned}
& X_{10}=-\bar{h}\left(\begin{array}{cc}
\frac{\Delta_{1} u_{x}}{\Delta_{1} u} & \frac{u_{x}\left(x_{0}, y_{0}\right)}{\Delta_{1} u} \\
0 & 0
\end{array}\right)+\mathcal{O}\left(\bar{h}^{2}\right) \\
& X_{20}=-\bar{k}\left(\begin{array}{cc}
\frac{\Delta_{1} u_{y}}{\Delta_{1} u} & \frac{u_{y}\left(x_{0}, y_{0}\right)}{\Delta_{1} u} \\
0 & 0
\end{array}\right)+\mathcal{O}\left(\bar{k}^{2}\right) \\
& X_{31}=X_{20}-\bar{h} \widehat{k X_{31}}+\mathcal{O}\left(\bar{h}^{2}, \bar{k}^{2}\right) \\
& =X_{20}-\bar{h} \bar{k}\left(\begin{array}{cc}
\frac{\Delta_{1} u_{x} \Delta_{1} u_{y}+\Delta_{1} u_{x y} \Delta_{1} u}{\left(\Delta_{1} u\right)^{2}} & h \frac{u_{y} \Delta_{1} u_{x}+u_{x y} \Delta_{1} u}{\left(\Delta_{1} u\right)^{2}} \\
0 & 0
\end{array}\right)+\mathcal{O}\left(\bar{h}^{2}, \bar{k}^{2}\right) \\
& X_{32}=X_{10}-\bar{h} \widehat{k X_{32}}+\mathcal{O}\left(\bar{h}^{2}, \bar{k}^{2}\right) \\
& =X_{10}-\bar{h} \bar{k}\left(\begin{array}{cc}
\frac{\Delta_{1} u_{x} \Delta_{1} u_{y}+\Delta_{1} u_{x y} \Delta_{1} u}{\left(\Delta_{1} u\right)^{2}} & h \frac{u_{x} \Delta_{1} u_{y}+u_{x y} \Delta_{1} u}{\left(\Delta_{1} u\right)^{2}} \\
0 & 0
\end{array}\right)+\mathcal{O}\left(\bar{h}^{2}, \bar{k}^{2}\right)
\end{aligned}
$$

where this defines $\widetilde{X_{31}}$ and $\widetilde{X_{32}}$. The local syzygy is $K_{31} K_{10}=K_{32} K_{20}$, and applying the Baker-Campbell-Hausdorff formula to this yields

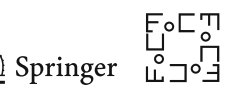




$$
X_{10}+X_{31}+\frac{1}{2}\left[X_{31}, X_{10}\right]=X_{32}+X_{20}+\frac{1}{2}\left[X_{32}, X_{20}\right]+\mathcal{O}\left(\bar{h}^{2}, \bar{k}^{2}\right) .
$$

The equation for the lower order terms simplifies to

$$
\widetilde{X_{32}}-\widetilde{X_{31}}=\left[X_{10}, X_{20}\right]
$$

This last equation is straightforward to verify. Finally, taking the limit as $h \rightarrow 0$, Eq. (26) yields the differential syzygy for $\rho$ evaluated on the jet bundle,

$$
\frac{\partial}{\partial y}\left(\rho_{x} \rho^{-1}\right)-\frac{\partial}{\partial x}\left(\rho_{y} \rho^{-1}\right)=\left[\rho_{y} \rho^{-1}, \rho_{x} \rho^{-1}\right]
$$

where recall $\rho$ on the jet bundle is given in Eq. (17).

Similar relationships exist when we take limits only in one of the variables, producing an evolution of discrete submanifolds. For example, if $p=2$ and $S$ has coordinates $(x, y)$, then if $K_{J, 2}^{i}=\rho_{J+e_{2}}\left(z_{J}^{i}\right) \rho\left(z_{J}\right)^{-1}$ and $N_{J}=\frac{d}{d x}\left(K_{J}\right) K_{J}^{-1}$, then when we take limits in the calculation in the proof of the theorem as $x_{J+e_{1}} \rightarrow x_{J}$ we have

$$
\frac{d}{d x}\left(K_{(J, 2)}\right) K_{(J, 2)}^{-1}=N_{J+e_{2}}-K_{(J, 2)} N_{J} K_{(J, 2)}^{-1}
$$

which is a mixed syzygy that often appears describing invariant evolutions of polygons in terms of coordinates in their moduli spaces, as in [42,47]. Among these evolutions one often finds completely integrable discretisations of well-known completely integrable PDEs. These results are really key to some of the applications in our next section.

\section{Applications}

\subsection{Application to the Design of a Lagrangian for a Variational Numerical Scheme for a Shallow Water System}

This example is motivated by the need for finite difference versions of variational shallow water problems which are invariant under the so-called particle relabelling symmetry. We consider the base space to have coordinates $(a, b, t)$, where $(a, b)$ is the fluid particle label at time $t=0$. The two-dimensional dependent variable space is $(x, y)=(x(a, b, t), y(a, b, t))$, which is the position of the fluid particle at time $t$, so that $(x(a, b, 0), y(a, b, 0))=(a, b)$. The particle relabelling action is given by

$$
g \cdot a=A(a, b), \quad g \cdot b=B(a, b), \quad A_{a} B_{b}-A_{b} B_{a}=1
$$

together with $g \cdot x=x, g \cdot y=y$ and $g \cdot t=t$. It can be seen that the particle relabelling group is the group of area preserving diffeomorphisms of the $(a, b)$ plane (or at least the domain of interest in the $(a, b)$ plane). Further, it is known that the 
invariants of this group action are $x, y, t$, and $\Delta=x_{a} y_{b}-x_{b} y_{a}$ and its derivatives under the invariant differential operators,

$$
\frac{\partial}{\partial t}, \quad \frac{\partial}{\partial x}=\frac{y_{b}}{\Delta} \frac{\partial}{\partial a}-\frac{y_{a}}{\Delta} \frac{\partial}{\partial b}, \quad \frac{\partial}{\partial y}=-\frac{x_{b}}{\Delta} \frac{\partial}{\partial a}+\frac{x_{a}}{\Delta} \frac{\partial}{\partial b} .
$$

The aim is to design a multispace version of the Lagrangian for variational shallow water problems, which have the form,

$$
\mathcal{L}[x, y]=\int L\left(x, y, x_{t}, y_{t}, x_{a} y_{b}-x_{b} y_{a}\right) \mathrm{d} a \mathrm{~d} b \mathrm{~d} t
$$

This family of Lagrangians is each invariant under translations in time, translations in both $a$ and $b$, rotations in the $(a, b)$ plane, and more generally, the full particle relabelling group. Noether's Theorem $[2,49,53]$ then yields conservation of energy, linear and angular momenta, and potential vorticity [1].

If we take the simplest corner lattice with base point $\left(a_{0}, b_{0}, t_{0}\right)$ to be

$$
\Gamma=\left\{\left(a_{0}, b_{0}, t_{0}\right),\left(a_{1}, b_{1}, t_{0}\right),\left(a_{2}, b_{2}, t_{0}\right),\left(a_{3}, b_{3}, t_{1}\right)\right\}
$$

then the (linear) interpolation of $x$ is given by

$$
x(a, b, t) \sim x\left(a_{0}, b_{0}, t_{0}\right)+\mathcal{M}\left(x_{a}\right)\left(a-a_{0}\right)+\mathcal{M}\left(x_{b}\right)\left(b-b_{0}\right)+\mathcal{M}\left(x_{t}\right)\left(t-t_{0}\right)
$$

where this defines the coefficients $\mathcal{M}\left(x_{K}\right)$, and we have

$$
\mathcal{M}\left(x_{a}\right)=\frac{1}{\mathcal{A}}\left|\begin{array}{ccc}
1 & x\left(a_{0}, b_{0}, t_{0}\right) & b_{0} \\
1 & x\left(a_{1}, b_{1}, t_{0}\right) & b_{1} \\
1 & x\left(a_{2}, b_{2}, t_{0}\right) & b_{2}
\end{array}\right|, \quad \mathcal{M}\left(x_{b}\right)=\frac{1}{\mathcal{A}}\left|\begin{array}{ccc}
1 & a_{0} & x\left(a_{0}, b_{0}, t_{0}\right) \\
1 & a_{1} & x\left(a_{1}, b_{1}, t_{0}\right) \\
1 & a_{2} & x\left(a_{2}, b_{2}, t_{0}\right)
\end{array}\right|
$$

and

$$
\mathcal{M}\left(x_{t}\right)=\frac{1}{\left(t_{1}-t_{0}\right) \mathcal{A}}\left|\begin{array}{llll}
1 & a_{0} & b_{0} & x\left(a_{0}, b_{0}, t_{0}\right) \\
1 & a_{1} & b_{1} & x\left(a_{1}, b_{1}, t_{0}\right) \\
1 & a_{2} & b_{2} & x\left(a_{2}, b_{2}, t_{0}\right) \\
1 & a_{3} & b_{3} & x\left(a_{3}, b_{3}, t_{1}\right)
\end{array}\right|
$$

where

$$
\mathcal{A}=\left|\begin{array}{lll}
1 & a_{0} & b_{0} \\
1 & a_{1} & b_{1} \\
1 & a_{2} & b_{2}
\end{array}\right|
$$

is the area, $\left|\left(a_{1}-a_{0}, b_{1}-b_{0}\right) \wedge\left(a_{2}-a_{0}, b_{2}-b_{0}\right)\right|$. The interpolant for $y$ is similar, with $y\left(a_{i}, b_{i}, t_{i}\right)$ replacing $x\left(a_{i}, b_{i}, t_{i}\right)$ in the above formulae. 
We consider the Lie group $\operatorname{SL}(2) \ltimes \mathbb{R}^{2}$ acting on the $(a, b)$ plane as the standard (right) equiaffine action,

$$
\left(\begin{array}{l}
a \\
b
\end{array}\right) \mapsto\left(\begin{array}{l}
g \cdot a \\
g \cdot b
\end{array}\right)=\left(\begin{array}{cc}
\delta & -\beta \\
-\gamma & \alpha
\end{array}\right)\left(\begin{array}{l}
a-\epsilon_{1} \\
b-\epsilon_{2}
\end{array}\right)
$$

so that $\left(\epsilon_{1}, \epsilon_{2}\right) \in \mathbb{R}^{2}$ is the translation vector, and $\alpha \delta-\beta \gamma=1$, and that $g \cdot x=x$, $g \cdot y$ and $g \cdot t=t$. This group is contained within the particle relabelling symmetry group, and is just big enough to obtain the area invariant, which we do next.

The induced action on the coefficients in the interpolants is given by, for example,

$$
g \cdot \mathcal{M}\left(x_{a}\right)=\frac{1}{\mathcal{A}}\left|\begin{array}{ccc}
1 & x\left(a_{0}, b_{0}, t_{0}\right) & g \cdot b_{0} \\
1 & x\left(a_{1}, b_{1}, t_{0}\right) & g \cdot b_{1} \\
1 & x\left(a_{2}, b_{2}, t_{0}\right) & g \cdot b_{2}
\end{array}\right|,
$$

noting that $g \cdot \mathcal{A}=\mathcal{A}$, indeed, $\mathcal{A}$ is an invariant as is easily seen.

We take the normalisation equations $g \cdot\left(a_{0}, b_{0}\right)=(0,0), g \cdot \mathcal{M}\left(x_{a}\right)=1, g$. $\mathcal{M}\left(x_{b}\right)=0$ and $g \cdot \mathcal{M}\left(y_{a}\right)=0$. Then the multispace frame is $\left(\epsilon_{1}, \epsilon_{2}\right)=\left(a_{0}, b_{0}\right)$ and

$$
\left(\begin{array}{cc}
\delta & -\beta \\
-\gamma & \alpha
\end{array}\right)=\left(\begin{array}{ll}
\mathcal{M}\left(x_{a}\right) & \mathcal{M}\left(x_{b}\right) \\
\mathcal{M}\left(y_{a}\right) & \mathcal{M}\left(y_{b}\right) \\
\mathcal{M}(\Delta) & \mathcal{M}(\Delta)
\end{array}\right)
$$

where $\mathcal{M}(\Delta)=\mathcal{M}\left(x_{a}\right) \mathcal{M}\left(y_{b}\right)-\mathcal{M}\left(x_{b}\right) \mathcal{M}\left(y_{a}\right)$. Evaluating the remaining coefficient on the frame, we obtain the invariant,

$$
\begin{aligned}
\left.I\left(\mathcal{M}\left(y_{b}\right)\right)\right) & =g \cdot \mathcal{M}\left(y_{b}\right) \mid \rho \\
& =\mathcal{M}(\Delta) \\
& =\frac{1}{\mathcal{A}}\left|\begin{array}{lll}
1 & x\left(a_{0}, b_{0}, t_{0}\right) & y\left(a_{0}, b_{0}, t_{0}\right) \\
1 & x\left(a_{1}, b_{1}, t_{1}\right) & y\left(a_{1}, b_{1}, t_{1}\right) \\
1 & x\left(a_{2}, b_{2}, t_{2}\right) & y\left(a_{2}, b_{2}, t_{2}\right)
\end{array}\right| .
\end{aligned}
$$

Calculating the continuum limit of $\mathcal{M}(\Delta)$ we obtain $x_{a} y_{b}-x_{b} y_{a}$, which is $\Delta$, the area invariant, as expected. Further, the continuum limit of the frame is

$$
\rho \rightarrow\left(\begin{array}{cc}
x_{a} & x_{b} \\
\frac{y_{a}}{x_{a} y_{b}-x_{b} y_{a}} & \frac{y_{b}}{x_{a} y_{b}-x_{b} y_{a}}
\end{array}\right) .
$$

This is the smooth frame obtained with the smooth limit of the normalisation equations, that is, $\left\{\widetilde{x_{a}}=1, \widetilde{x_{b}}=0, \widetilde{y_{a}}=0\right\}$.

We observe that both $\mathcal{M}\left(x_{t}\right)$ and $\mathcal{M}\left(y_{t}\right)$ are invariant under the equiaffine action. Thus, we propose the multispace analogue of the Lagrangian (27) to be

$$
\mathcal{M}(\mathcal{L}[x, y])=\sum_{\Gamma} L\left(x, y, \mathcal{M}\left(x_{t}\right), \mathcal{M}\left(y_{t}\right), \mathcal{M}(\Delta)\right) \mathcal{A}\left(t_{1}-t_{0}\right)
$$


Fig. 17 Length one lattices stacked as a mesh, for a finite difference variational problem. Shown here is single time slice for the shallow water problem

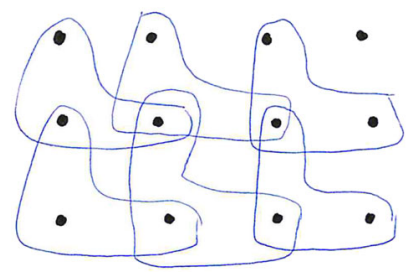

where the sum is over all corner lattices stacked into a mesh, as in Fig. 17. The factor $\mathcal{A}\left(t_{1}-t_{0}\right)$ is the multispace approximation of the volume form, $\mathrm{d} a \mathrm{~d} b \mathrm{~d} t$, and is needed to obtain the correct continuum limits for the conservation laws for energy and the linear momenta.

Finite difference Euler Lagrange equations and Noether's conservation laws can be calculated in the standard way [23,32,33]; the details and the results of this calculation will be explored elsewhere. It is interesting to observe that the multispace Lagrangian, (28) is invariant under a discrete analogue of the particle relabelling symmetry. Indeed, looking at Fig. 17, one can use a different element of $\operatorname{SL}(2) \ltimes \mathbb{R}^{2}$ on each basepoint of each individual corner lattice, inducing an action on the whole of the corner lattice, provided that certain consistency conditions hold, specifically, that if a vertex is in the intersection of two corner lattices, that their image under the two different group elements is the same. The Lagrangian (28) is clearly invariant under this discretisation of the particle relabelling group, the discretisation being a subgroup of $\left(\operatorname{SL}(2) \ltimes \mathbb{R}^{2}\right)^{R}$ where $R$ is the number of corner lattices on a time slice of the mesh. Using this symmetry group to study the Lagrangian requires relaxing the assumption that we use the same action of the group at every lattice (i.e. we relax the assumption of the product action). This would require an extension of the theory developed in this paper, which we consider elsewhere.

\subsection{Discretisations of Completely Integrable PDEs}

The geometry of curves and surfaces have been linked to integrable systems repeatedly in the literature, see $[24,45,58]$, for example. A drawback of the application of the results in this paper to finding completely integrable discretisations of completely integrable systems is that one needs to choose a type of approximation (forward, backwards, linear or higher order, etc) a priori to find the limit. On the other hand, any two choices of discrete moving frames (be the one associated with a certain type of limit or any different one) will always be associated by a gauge transformation. This means that if one finds a discrete integrable system associated with any given choice of moving frame, one might be able to relate it to a different choice and perhaps link it to the continuous case. This was done in [47], where the authors found discrete integrable systems that were the discretisation of Adler-Gel'fand-Dikii integrable evolutions, both of them linked to the projective geometry of curves and polygons. The authors of [47] also found a way to obtain two Hamiltonian structures associated with the discrete system through a reduction process, a process that was later extended to other semisimple homogeneous spaces in [46]. Different approaches were used, for

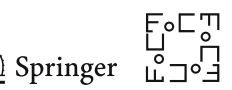


example in [3] and in other works of these authors to construct completely integrable discretisations of integrable systems with the use of lattice models in Euclidean, projective and conformal geometry. Their approach is quite different from the one used in [47] and in this paper, in that they choose lattices with different geometric properties to achieve discretisations. The following are just some examples of the connection between continuous and discrete models, in the equi-centro-affine and the Euclidean space. Both of these examples use mixed multispace discrete/continuous models, and although the first example only uses discrete coordinates to construct the multispace moving frame, the second example will make full use of both.

\subsubsection{Integrable Discretisations of Boussinesq Equations}

In this example we make use of mixed differential/difference coordinates in the multispace. For reasons that will become clear later, we will also assume that the lattice variety has a monodromy in the discrete variable (a global property). That is, $u_{n+T}(t)=\mathbf{m} \cdot u_{n}(t)$ for any $n \in \mathbb{Z}$, with $T$ the period and some monodromy $\mathbf{m} \in \mathrm{SL}(3, \mathbb{R})$. This ensures that the invariants will be $T$-periodic in $n$.

Continuous Case First we describe the situation when we are in continuous jets with two parameters $(x, t)$. It is well known (see for example [6]) that the Boussinesq equations

$$
\left(q_{0}\right)_{t}+\frac{1}{6} q_{1}^{\prime \prime \prime}+\frac{2}{3} q_{1} q_{1}^{\prime}=0, \quad\left(q_{1}\right)_{t}-2 q_{0}^{\prime}=0
$$

where the prime denotes $d / d x$, can be obtained as the evolution induced on equicentro-affine curvatures by a certain evolution of curves. Let our manifold be $M=\mathbb{R}^{3}$ with $G=\operatorname{SL}(3, \mathbb{R})$ acting linearly on it. Within $M$ consider parametrised surfaces on $(x, t)$. Thus, in this example $u(x, t) \in \mathbb{R}^{3}$, unlike in previous examples when we consider graphs of the form $(x, y, u(x, y))$. Hopefully this will not confuse the reader. We will define the following cross section:

$$
\rho \cdot u=e_{3}, \quad \rho \cdot u^{\prime}=e_{2}, \quad \rho \cdot u^{\prime \prime}=d e_{1}
$$

where $e_{i}$ are the standard unit vectors in $\mathbb{R}^{3}$. Clearly $d=\operatorname{det}\left(u, u^{\prime}, u^{\prime \prime}\right)$. This defines uniquely a right moving frame whose left companion is given by

$$
\rho^{-1}=\left(\frac{1}{d} u^{\prime \prime}, u^{\prime}, u\right) \text {. }
$$

Let us assume that

$$
d=\operatorname{det}\left(u^{\prime \prime}, u^{\prime}, u\right)=1,
$$

that is we will parametrise the surface so that the curves associated with $t$ fixed are parametrised by the equi-centro-affine arc-length (these curves are in one-to-one correspondence with projective curves, see [6]. In that case the left $x$-Maurer-Cartan matrix associated with it is given by 


$$
Q=\rho\left(\rho^{-1}\right)^{\prime}=\left(\begin{array}{lll}
0 & 1 & 0 \\
k_{1} & 0 & 1 \\
k_{2} & 0 & 0
\end{array}\right)
$$

where $u^{\prime \prime \prime}=k_{1} u^{\prime}+k_{2} u$. Next we will gauge this frame to a different left frame $\widehat{\rho}=\rho^{-1} g$ by the element

$$
g=\left(\begin{array}{ccc}
1 & 0 & 0 \\
0 & 1 & 0 \\
-k_{1} & 0 & 1
\end{array}\right)
$$

The resulting $x$-Maurer-Cartan matrix is given by

$$
K=g^{-1} g^{\prime}+g^{-1} Q g=\left(\begin{array}{lll}
0 & 1 & 0 \\
0 & 0 & 1 \\
b & a & 0
\end{array}\right)
$$

where $a=k_{1}$ and $b=k_{2}-k_{1}^{\prime}$. Gauging the system can be seen as changing the coordinates, the results can always be gauged back to the original setting.

We will next consider the syzygy

$$
u_{t}-u^{\prime \prime}+\frac{2}{3} a u=0
$$

which describes a precisely chosen evolutionary equation for curves whose flow will be tracing our parametrised surface. With this condition, the left $t$-Maurer-Cartan matrix is easily seen to be given by

$$
N=\widehat{\rho}^{-1} \widehat{\rho}_{t}=\left(\begin{array}{ccc}
-\left(w_{1}+1 / 3 a\right) & w_{0} & 1 \\
v_{1} & w_{1} & 0 \\
v_{2} & w_{2} & \frac{1}{3} a
\end{array}\right)
$$

for some entries $v_{i}, w_{i}$. The local basic syzygies (or the compatibility condition between $x$ and $t$ ) are given by

$$
K_{t}=N_{x}+[K, N]
$$

and they can be used to solve for $N$ so that

$$
w_{0}=0, \quad w_{1}=\frac{1}{3} a, \quad w_{2}=b+\frac{1}{3} a^{\prime}, \quad v_{1}=b+\frac{2}{3} a^{\prime}, \quad v_{2}=-b^{\prime}-\frac{2}{3} a^{\prime \prime} .
$$

We can further find two more syzygies given by

$$
a_{t}-2 b^{\prime}-a^{\prime \prime}=0, \quad b_{t}+\frac{2}{3} a^{\prime \prime \prime}+b^{\prime \prime}-\frac{2}{3} a^{\prime} a .
$$

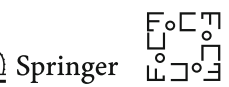


This system of equations is equivalent to the Boussinesq equation. Indeed, if $a=q_{1}$ and $b=\frac{1}{2} q_{1}^{\prime}-q_{0}$, we have

$$
\left(q_{0}\right)_{t}+\frac{1}{6} q_{1}^{\prime \prime \prime}+\frac{2}{3} q_{1} q_{1}^{\prime}=0, \quad\left(q_{1}\right)_{t}-2 q_{0}^{\prime}=0
$$

which is the standard Boussinesq equation.

Multispace Case Assume now that we move in the multispace away from a continuous jet to a mixed discrete/continuous multispace submanifold, where $x$ is now discrete and $t$ is continuous. Let us choose lattices containing $\left(x_{n}, t\right),\left(x_{n+1}, t\right),\left(x_{n+2}, t\right)$ and such that $x_{r+1}-x_{r}=\Delta x_{r}=c$ is constant for any $r$. That is, we will restrict to lattices with sides of equal length. The cross section (29) evaluated on lattices of this form will be given by

$$
\rho \cdot u_{n}=e_{3}, \quad \rho \cdot\left(u_{n+1}-u_{n}\right) / c=e_{2}, \quad \rho \cdot\left(\frac{1}{c^{2}}\left(u_{n+2}-2 u_{n+1}+u_{n}\right)\right)=e_{1}
$$

where $1=\left(1 / c^{3}\right) \operatorname{det}\left(u_{n+2}, u_{n+1}, u_{n}\right)$ and $u_{i}=u\left(x_{i}, t\right)$. The left moving frame associated with this cross section is given by

$$
\begin{aligned}
\rho^{-1} & =\left(\frac{1}{c^{2}}\left(u_{n+2}-2 u_{n+1}+u_{n}\right), \frac{1}{c}\left(u_{n+1}-u_{n}\right), u_{n}\right) \\
& =\left(\begin{array}{llll}
u_{n+2} & u_{n+1} & u_{n}
\end{array}\right)\left(\begin{array}{ccc}
c^{-2} & 0 & 0 \\
-2 c^{-2} & c^{-1} & 0 \\
c^{-2} & -c^{-1} & 1
\end{array}\right)
\end{aligned}
$$

which can clearly be gauged to

$$
\eta=\left(u_{n+2}, u_{n+1}, u_{n}\right) .
$$

The multispace subspace (30), when restricted to our partially coalesced lattices, becomes

$$
\operatorname{det}\left(u_{n+2}, u_{n+1}, u_{n}\right)=c^{3}
$$

for all $n$. Let us introduce one last gauge by the matrix

$$
g=\left(\begin{array}{ccc}
1 & 0 & 0 \\
-a_{n-1} & 1 & 0 \\
0 & 0 & 1
\end{array}\right)
$$

where $a_{n}$ is to be found. If $u_{n+3}=k_{1}^{n} u_{n+2}+k_{2}^{n} u_{n+1}+u_{n}$, then, the discrete $x$ Maurer-Cartan matrix associated with $\rho=\eta g$ is given by

$$
K_{n}=\left(\begin{array}{ccc}
0 & 1 & 0 \\
b_{n} & a_{n} & 1 \\
1 & 0 & 0
\end{array}\right)
$$


where $b_{n}=k_{2}^{n}$ and $a_{n}=k_{1}^{n-1}$. As before, the $t$-Maurer-Cartan matrix is given by

$$
N_{n}=\widehat{\rho}_{n}^{-1}\left(\widehat{\rho}_{n}\right)_{t}=\left(\begin{array}{ccc}
-\left(w_{1}^{n}+r_{2}^{n}\right) & w_{0}^{n} & r_{0}^{n} \\
v_{1}^{n} & w_{1}^{n} & r_{1}^{n} \\
v_{2}^{n} & w_{2}^{n} & r_{2}^{n}
\end{array}\right)
$$

and the local basic syzygy is

$$
K_{n}^{-1}\left(K_{n}\right)_{t}=N_{n+1}-K_{n}^{-1} N_{n} K_{n}
$$

This syzygy solves for $N_{n}$ in terms of $r_{i}$

$$
\begin{aligned}
& w_{0}^{n}=r_{1}^{n+1} ; \quad w_{1}^{n}=r_{2}^{n+1}+a_{n} r_{1}^{n+1}+b_{n} r_{0}^{n+1} ; \quad w_{2}^{n}=r_{0}^{n+1} ; \\
& v_{1}^{n}=r_{0}^{n-1}+b_{n-1} r_{1}^{n} ; \quad v_{2}^{n}=r_{1}^{n+2}-a_{n} r_{0}^{n+1},
\end{aligned}
$$

and it provides the condition for preserving the restriction to the multispace submanifold (32), namely

$$
r_{2}^{n+2}+r_{2}^{n+1}+r_{2}^{n}+a_{n+1} r_{1}^{n+2}+b_{n+1} r_{0}^{n+2}+b_{n} r_{0}^{n+1}=0
$$

If the map $r_{2}^{n} \rightarrow r_{2}^{n+2}+r_{2}^{n+1}+r_{2}^{n}=\left(\mathcal{T}^{2}+\mathcal{T}+1\right) r_{2}^{n}$ is invertible (which is true if $N \neq 3 s$ for any $s$ as shown in [47]), this condition solves for $r_{2}^{n}$ in terms of $r_{1}^{n}$ and $r_{0}^{n}$. The syzygy also describes $\left(a_{n}\right)_{t}$ and $\left(b_{n}\right)_{t}$ to satisfy

$$
\begin{aligned}
\left(a_{n}\right)_{t}= & \left(1+a_{n} b_{n+1}\right) r_{0}^{n+2}-\left(a_{n} b_{n} r_{0}^{n+1}+r_{0}^{n-1}\right) \\
& +\left(b_{n}+a_{n} a_{n+1}\right) r_{1}^{n+2}-\left(b_{n-1} r_{1}^{n}+a_{n}^{2} r_{1}^{n+1}\right)+a_{n} r_{2}^{n+2}-a_{n} r_{2}^{n} \\
\left(b_{n}\right)_{t}= & r_{1}^{n+3}-\left(b_{n} a_{n+1} r_{1}^{n+2}+r_{1}^{n}\right)-\left(a_{n+1}+b_{n} b_{n+1}\right) r_{0}^{n+2} \\
& -b_{n}^{2} r_{0}^{n+1}+a_{n} r_{0}^{n}-\left(b_{n} r_{2}^{n+2}+2 b_{n} r_{2}^{n+1}\right) .
\end{aligned}
$$

If further we impose the syzygy

$$
\left(u_{n}\right)_{t}+\frac{1}{b_{n-1}}\left(u_{n+2}-a_{n} u_{n+1}\right)+\frac{2}{3} u_{n}=0
$$

then we can see that $r_{0}^{n}=\frac{1}{b_{n-1}}, r_{1}^{n}=0$ and $r_{2}^{n}=-\left(\mathcal{T}^{2}+\mathcal{T}+1\right)^{-1}(\mathcal{T}+1) \frac{b_{n}}{b_{n}}=-\frac{2}{3}$ is the solution of (33) for these choices. Then

$$
\begin{aligned}
\left(a_{n}\right)_{t} & =\frac{1}{b_{n+1}}-\frac{1}{b_{n-2}} \\
\left(b_{n}\right)_{t} & =-\frac{a_{n+1}}{b_{n+1}}+\frac{a_{n}}{b_{n-1}}
\end{aligned}
$$

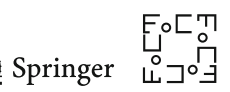


and the changes

$$
\alpha_{n}=-\frac{1}{b_{n} b_{n+1} b_{n+2}}, \quad \beta_{n}=-\frac{a_{n+1}}{b_{n} b_{n+1}}
$$

transform this equation in the integrable discretisation of Boussinesq

$$
\begin{aligned}
& \left(\alpha_{n}\right)_{t}=\alpha_{n}\left(\beta_{n+2}-\beta_{n-1}\right) \\
& \left(\beta_{n}\right)_{t}=\alpha_{n-1}-\alpha_{n}+\beta_{n}\left(\beta_{n+1}-\beta_{n-1}\right) .
\end{aligned}
$$

This system appears in [25].

It is not clear to us how to systematically connect integrable discrete systems and evolutions of polygons as given by (34). In the continuous case there is a general link between Hamiltonian evolutions at the level of the invariants and evolution of curves on geometric manifolds which are homogeneous of the form $G / H$ or $\left(G \rtimes \mathbb{R}^{n}\right) / G$ with $G$ semisimple (see $[43,44]$ ), but the situation in the discrete case is not so clear in general. In particular, the syzygies (31) and (34) are not the restriction of the same syzygy on different points in the multispace, even when we account for all the different changes introduced by gauges. Still, it is widely known that certain evolutions of polygons result in completely integrable discrete systems (see, for example, [4] in the Euclidean case and [3] in more cases with further restrictions on the lattices). The multispace allows us to construct geometrically without the need to account for the limits. We include one more example along these lines and further use of multispace in this area will appear elsewhere.

\subsubsection{Discretisation of the Sine-Gordon Equation}

It is well known that the Codazzi-Mainardi equations for Euclidean surfaces in $\mathbb{R}^{3}$ with constant negative Gauss curvature includes a Sine-Gordon equation, a wellknown completely integrable system, that decouples from the rest of the determining equations for the surface. The Codazzi-Mainardi equations are simply syzygies for a well-chosen moving frame, hence using the multispace framework we will be able to find a discretisation of the Sine-Gordon equation with strong geometric meaning as determining mixed lattice/surfaces with negative Gauss curvature. It is not clear to us whether the discretisation below is completely integrable as it becomes part of a system of equations defining the lattice/surface, rather than decoupling to discretise SineGordon individually. A discrete Sine-Gordon equation on lattices was also defined in [3], although the conditions that the authors imposed on their lattices are not impose here. Further study on the connection between both approaches will appear elsewhere.

We review the continuous case first.

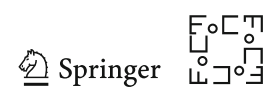




\subsubsection{Sine-Gordon as Syzygy of Euclidean Surfaces with Constant Negative Curvature}

Let $G$ be the Euclidean group represented as the subgroup of $\operatorname{GL}(4, \mathbb{R})$

$$
g=\left(\begin{array}{ll}
1 & 0 \\
v & \Theta
\end{array}\right)
$$

where $v \in \mathbb{R}^{3}$ and $\Theta \in \mathrm{SO}(3)$. The group acts in $\mathbb{R}^{3}$ with the standard action $g \cdot u=$ $\Theta u+v$ which coincides with the one induced by $g\left(\begin{array}{l}1 \\ u\end{array}\right)$. Let $u(x, y)$ be a parametrised surface and assume that $x$ and $y$ are normalised to measure the arc-length in the $x$ and $y$ direction. That is, assume that $\left\|u_{x}\right\|=\left\|u_{y}\right\|=1$. Let us define a moving frame through the normalisations

$$
\rho \cdot u=0, \quad \rho \cdot u_{x}=e_{1}, \quad \rho \cdot u_{y}=\cos \alpha e_{1}+\sin \alpha e_{2}
$$

where $\alpha$ is the angle formed by $u_{x}$ and $u_{y}$. Solving the equations we obtain that $v=-\Theta u$ and

$$
\Theta^{T}=\left(u_{x} \frac{1}{\sin \alpha}\left(u_{y}-\cos \alpha u_{x}\right) n\right)
$$

where $n=\frac{1}{\sin \alpha}\left(u_{x} \times u_{y}\right)$ is the standard normal unit vector determined by the parametrisation. Using the traditional notation $u_{x x}=\Gamma_{11}^{1} u_{x}+\Gamma_{11}^{2} u_{y}+e n, u_{x y}=$ $\Gamma_{12}^{1} u_{x}+\Gamma_{12}^{2} u_{y}+f n, u_{y y}=\Gamma_{22}^{1} u_{x}+\Gamma_{22}^{2} u_{y}+g n$, we can write the Maurer-Cartan matrices as

$$
\begin{aligned}
& \widehat{N}=\rho_{x} \rho^{-1}=\left(\begin{array}{cc}
0 & 0 \\
-(\Theta u)_{x} \Theta_{x}
\end{array}\right)\left(\begin{array}{cc}
1 & 0 \\
u & \Theta^{T}
\end{array}\right)=\left(\begin{array}{cc}
0 & 0 \\
-e_{1} & \Theta_{x} \Theta^{T}
\end{array}\right) \\
& \widehat{K}=\rho_{y} \rho^{-1}=\left(\begin{array}{cc}
0 & 0 \\
-\cos \alpha e_{1}-\sin \alpha e_{2} & \Theta_{y} \Theta^{T}
\end{array}\right)
\end{aligned}
$$

where

$$
\begin{gathered}
N=\Theta_{x} \Theta^{T}=\left(\begin{array}{ccc}
0 & \sin \alpha \Gamma_{11}^{2} & e \\
-\sin \alpha \Gamma_{11}^{2} & 0 & \frac{1}{\sin \alpha}(f-\cos \alpha e) \\
-e & \frac{-1}{\sin \alpha}(f-\cos \alpha e) & 0
\end{array}\right) \\
K=\Theta_{y} \Theta^{T}=\left(\begin{array}{ccc}
0 & \sin \alpha \Gamma_{12}^{2} & f \\
-\sin \alpha \Gamma_{12}^{2} & 0 & \frac{1}{\sin \alpha}(g-\cos \alpha f) \\
-f & \frac{-1}{\sin \alpha}(g-\cos \alpha f) & 0
\end{array}\right) .
\end{gathered}
$$


Substituting these values in the local syzygy $\widehat{K}_{x}=\widehat{N}_{y}+[\widehat{K}, \widehat{N}]$ and selecting the $\mathbb{R}^{3}$ component, we get the equation

$$
\begin{aligned}
0= & \left(\begin{array}{c}
-\cos \alpha \\
-\sin \alpha \\
0
\end{array}\right)_{x}-\left(\begin{array}{c}
0 \\
-\sin \alpha \Gamma_{12}^{2} \\
-f
\end{array}\right)+\cos \alpha\left(\begin{array}{c}
0 \\
-\sin \alpha \Gamma_{11}^{2} \\
-e
\end{array}\right) \\
& +\sin \alpha\left(\begin{array}{c}
\sin \alpha \Gamma_{11}^{2} \\
0 \\
\frac{-1}{\sin \alpha}(f-\cos \alpha e)
\end{array}\right) .
\end{aligned}
$$

The last entry is trivial, the first solves for the value

$$
\Gamma_{11}^{2}=\frac{-1}{\sin \alpha} \alpha_{x}
$$

while the second one simplifies to $\Gamma_{12}^{2}=0$, whenever $\sin \alpha \neq 0$. The $\operatorname{SO}(3)$ portion of the syzygy is given by

$$
K_{x}=N_{y}+[K, N] .
$$

If we write down the equation that does not involve derivatives of the second fundamental form (the equations defined by the $(1,2)$ entry), we have

$$
\left(\sin \alpha \Gamma_{11}^{2}\right)_{y}=\frac{-f}{\sin \alpha}(f-\cos \alpha e)+\frac{e}{\sin \alpha}(g-\cos \alpha f),
$$

which becomes

$$
\alpha_{x y}=-\frac{1}{\sin \alpha}\left(e g-f^{2}\right)
$$

If $\mathbf{K}$ is the Gauss curvature, we know that $\mathbf{K}=\frac{e g-f^{2}}{E G-F^{2}}$, where $E=\left\|u_{x}\right\|=G=$ $\left\|u_{y}\right\|=1$ and $F=u_{x} \cdot u_{y}=\cos \alpha$. Thus, the equation becomes

$$
\alpha_{x y}=-\sin \alpha \mathbf{K}
$$

which is the Sine-Gordon equation whenever $\mathbf{K}$ is constant and negative.

Notice that this equation is not enough to determine the surface. Indeed, solving for $\alpha$ only determines the first fundamental form (or metric), and the knowledge of the Gauss curvature does not suffice to determine the second fundamental form. Indeed, one would need two more equations to do so, given by the two remaining entries $(1,3)$ and $(2,3)$ of the $\mathrm{SO}(3)$ portion of the local syzygy, i.e. (38). Thus, the surface is determined upon solving a system of 3 equations, one of which decouples and is equal to Sine-Gordon. 


\subsubsection{A Differential-Difference Sine-Gordon Evolution as a Syzygy of a Mixed Smooth-Discrete Lattice}

Assume we have a smooth family of polygons, or a mixed (1, 1)-lattice (one continuous direction and one discrete one) of the form $y_{r}, y_{r+1}$ in the $y$ direction and continuous 1 -jet in the $x$ direction. As far as we use the same multispace cross section, we will have guaranteed that discrete invariant data approximates the continuous one. Thus, consider the transverse section

$$
\begin{aligned}
& \rho_{r} \cdot u_{r}=\rho_{r} \cdot u\left(x, y_{r}\right)=0 ; \quad \rho_{r} \cdot{ }_{p} u_{x}=\left\|u_{x}\right\| e_{1} \\
& \frac{1}{y_{1}-y_{0}}\left(\rho_{r} \cdot \mathcal{T} u_{r}-\rho_{r} \cdot u_{r}\right)=\frac{\left\|\Delta u_{r}\right\|}{\Delta y_{0}} w_{r}
\end{aligned}
$$

where $\rho_{r}$ is as in $(35) ; \mathcal{T} u_{r}=u_{r+1}=u\left(x, \mathcal{T} y_{r}\right)=u\left(x, y_{r+1}\right) ; \cdot p$ is the prolonged action given by $\rho_{r} \cdot p u_{x}=\Theta u_{x}$; and $w_{r}$ is a unit vector with $w_{r}=\cos \alpha_{r} e_{1}+\sin \alpha_{r} e_{2}$ where $\alpha_{r}=\alpha_{r}(x)$ is the angle between $u_{x}$ and $\Delta u_{r}, \Delta u_{r}=(\mathcal{T}-1) u_{r}$. From here, the multispace cross section defines $v_{r}=-\Theta_{r} u_{r}, \Theta_{r} u_{x}=\left\|u_{x}\right\| e_{1}$ and $\Theta_{r} \Delta u_{r}=$ $\left\|\Delta u_{r}\right\| w_{r}$. With these choices the right moving frame becomes

$$
\rho_{r}=\left(\begin{array}{cc}
1 & 0 \\
v_{r} & \Theta_{r}
\end{array}\right), \quad v_{r}=-\Theta_{r} u_{r}
$$

with

$$
\begin{aligned}
\Theta_{r}^{T} & =\left(\begin{array}{lll}
t_{r} & n_{r} & b_{r}
\end{array}\right) \\
t_{r} & =\frac{\left(u_{r}\right)_{x}}{\left\|\left(u_{r}\right)_{x}\right\|} ; \quad n_{r}=\frac{1}{\sin \alpha_{r}}\left(\frac{\Delta u_{r}}{\left\|\Delta u_{r}\right\|}-\cos \alpha_{r} t_{r}\right) ; \quad b_{r}=t_{r} \times n_{r} .
\end{aligned}
$$

From now on, and for convenience, we will drop the subindex to denote position unless the situation is confusing, indicating a change in position by the application of the shift operator $\left(\mathcal{T}^{k} u=u_{r+k}\right)$. We will calculate the left Maurer-Cartan matrices, the more geometrically significant one (those in the continuous case are right ones). The left Maurer-Cartan matrices are given by

$$
\widehat{K}=\rho \mathcal{T} \rho^{-1}=\left(\begin{array}{cc}
1 & 0 \\
-\Theta u & \Theta
\end{array}\right)\left(\begin{array}{cc}
1 & 0 \\
\mathcal{T} u & \mathcal{T} \Theta^{T}
\end{array}\right)=\left(\begin{array}{cc}
1 & 0 \\
\|\Delta u\| w & K
\end{array}\right)
$$

where $K=\Theta \mathcal{T} \Theta^{-1}$; and by

$$
\widehat{N}=\rho\left(\rho^{-1}\right)_{t}=\left(\begin{array}{cc}
1 & 0 \\
-\Theta u & \Theta
\end{array}\right)\left(\begin{array}{cc}
0 & 0 \\
u_{x} & \left(\Theta^{T}\right)_{x}
\end{array}\right)=\left(\begin{array}{cc}
0 & 0 \\
\left\|u_{x}\right\| e_{1} & N
\end{array}\right)
$$

where $N=\Theta\left(\Theta^{-1}\right)_{x}$. The local syzygies are given by

$$
(\widehat{K})_{x}=\widehat{K} \mathcal{T} \widehat{N}-\widehat{N} \widehat{K}
$$

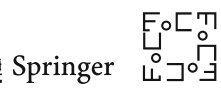


As we did in the continuous case, and to ensure convergence, we will restrict to the submanifold of the multispace defined locally by

$$
\left\|u_{x}\right\|=1 \quad \frac{\left\|\Delta u_{r}\right\|}{\left|\Delta y_{r}\right|}=1
$$

For simplicity we will restrict further to those lattices where $\left|\Delta y_{r}\right|=\epsilon$ for all $r$ (and hence $\left.\left\|\Delta u_{r}\right\|=\epsilon\right)$.

Equation (39) breaks into two equations, namely

$$
\begin{aligned}
& w_{t}=K e_{1}-N w-e_{1} \\
& K_{t}=K \mathcal{T} N-N K .
\end{aligned}
$$

Assume $K$ factorises as

$$
K=Y\left(\begin{array}{ll}
\Omega & 0 \\
0 & 1
\end{array}\right)
$$

with $\Omega \in \mathrm{SO}(2)$, for some $Y=\exp \left(\begin{array}{cc}0 & y \\ -y^{T} & 0\end{array}\right)$ (this is always possible when $\mathcal{T} \Theta$ is closed enough to $\Theta$ so that $K$ is closed enough to the identity). Assume further that

$$
\Omega=\left(\begin{array}{cc}
\cos k & -\sin k \\
\sin k & \cos k
\end{array}\right)=(v \widehat{v})
$$

$v$ and $\widehat{v}$ denoting the two columns of $\Omega$. We will denote with a hat the transformation $\widehat{v}=\left(\begin{array}{cc}0 & -1 \\ 1 & 0\end{array}\right) v=J v$, and so $\widehat{w}=J w$. Finally, denote $N$ by

$$
N=\left(\begin{array}{cc}
\Lambda & z \\
-z^{T} & 0
\end{array}\right), \quad \Lambda=\left(\begin{array}{cc}
0 & v \\
-v & 0
\end{array}\right), \quad z=\left(\begin{array}{l}
z_{1} \\
z_{2}
\end{array}\right)
$$

With this notation (40) can be rewritten and simplified to equations

$$
\begin{aligned}
-s y \cdot v & =z \cdot w \\
w \cdot e_{1} & =w \cdot v+c y \cdot v y \cdot w \\
\alpha_{x} & =-v+\frac{1}{\sin \alpha}\left(1-\cos k-c y \cdot v y_{2}\right)
\end{aligned}
$$

where $s=\frac{1}{\|y\|} \sin \|y\|, c=\frac{1}{\|y\|^{2}}(\cos \|y\|-1)$, and as usual $y^{T}=\left(y_{1}, y_{2}\right)$.

The remaining three equations that will determine the lattice/surface are given by the three entries in the $\mathfrak{s o ( 3 )}$ component (41). We will only reproduce the portion corresponding to Sine-Gordon, that is the $(2,1)$ entry of $(41)$. After some long, but straightforward algebraic manipulations, the equation becomes

$$
k_{x}+c \operatorname{det}\left(y, y_{x}\right)=\mathcal{T} v-\cos \|y\| v+s \operatorname{det}(y, z) .
$$


Looking at (37) and (36), and comparing it to (42) and (43), we see that

$$
s \operatorname{det}(y, z)
$$

discretises the determinant of the first two entries of the last column in both (37) and (36). That is, $s \operatorname{det}(y, z)$ discretises

$$
\operatorname{det}\left(\begin{array}{cc}
f & e \\
\frac{1}{\sin \alpha}(g-\cos \alpha f) & \frac{1}{\sin \alpha}(f-\cos \alpha e)
\end{array}\right)=-\sin \alpha \mathbf{K}
$$

were $\mathbf{K}$ is the Gauss curvature of the surface. Therefore, we can define

$$
\mathbf{K}=-\frac{s}{\sin \alpha} \operatorname{det}(y, z)=-\frac{\sin \|y\|}{\|y\| \sin \alpha} \operatorname{det}(y, z)
$$

to be the discrete Gauss curvature for the lattice/surface. Then, (47) becomes

$$
k_{x}+c \operatorname{det}\left(y, y_{x}\right)=\mathcal{T} v-\cos \|y\|-\sin \alpha \mathbf{K} .
$$

This will be a discretisation of Sine-Gordon, together with the other equations in the system.

Acknowledgements The authors would like to thank Peter Olver and Amos Ron for discussions, and especially the two referees whose lengthy and detailed comments greatly improved this paper.

Open Access This article is distributed under the terms of the Creative Commons Attribution 4.0 International License (http://creativecommons.org/licenses/by/4.0/), which permits unrestricted use, distribution, and reproduction in any medium, provided you give appropriate credit to the original author(s) and the source, provide a link to the Creative Commons license, and indicate if changes were made.

\section{Appendix: Equicontinuous Families of Discrete Frames}

In this Appendix, we use the Arzela-Ascoli Theorem to give a general convergence result for an equicontinuous family of moving frames. This provides a rigorous foundation to a variety of examples involving the discretisation of a smooth frame.

Let $M$ be a manifold, and let $G$ be a Lie group with local metric $d$. The set $G^{M}$ consists of all continuous maps from $M$ to $G$, and we give it the compact-open topology, defined as that generated by finite intersections of the so-called subbasic sets,

$$
(A, V)=\left\{f \in G^{M} \mid f(A) \subset V\right\}
$$

where $A \subset M$ is open and $V \subset G$ is compact. A sequence of maps converging in this topology is uniformly convergent on compact subsets.

Definition 6.1 A family $\mathcal{F} \subset G^{M}$ is said to be equicontinuous at $y_{0} \in M$ if for all $\epsilon>0$ there exists a neighbourhood $\mathcal{U}\left(y_{0}\right) \subset M$ such that for all $\rho \in \mathcal{F}$,

$$
\rho\left(\mathcal{U}\left(y_{0}\right)\right) \subset B\left(\rho\left(y_{0}\right), \epsilon\right)=\left\{g \in G \mid d\left(g, \rho\left(y_{0}\right)\right)<\epsilon\right\} .
$$

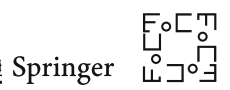


Theorem 6.2 Suppose that a family of left (resp. right) moving frames $\mathcal{F} \subset G^{M}$ satisfies the following:

(1) $\mathcal{F}$ is equicontinuous on $M$, and

(2) the set

$$
\{\rho(y) \mid \rho \in \mathcal{F}\}
$$

has compact closure for each $y$.

Then $\overline{\mathcal{F}}$ is a compact and equicontinuous family of moving frames.

Proof We give the proof for $\mathcal{F}$ a family of left frames, the proof for right frames is analogous. The conditions of the Theorem are precisely those of the Arzela-Ascoli Theorem, [15, XII, Theorem 6.4], which yields that the family $\overline{\mathcal{F}}$ is compact and equicontinuous. We need only show that its elements are also equivariant with respect to the group action on $M$. Fix $y \in M$ and $f \in \overline{\mathcal{F}}$, and let $\epsilon>0$ be given. By the definition of the closure of the set $\mathcal{F}$ and the continuity of the group action, there is a $\rho \in \mathcal{F}$ and a neighbourhood $U$ of the identity $e \in G$ such that for $g \in U$ we have both $d(f(g \cdot y), \rho(g \cdot y))<\frac{1}{2} \epsilon$ and $d(g \cdot f(y), g \cdot \rho(y))<\frac{1}{2} \epsilon$. Then

$$
\begin{aligned}
d(g \cdot f(y), f(g \cdot y)) & <d(f(g \cdot y), \rho(g \cdot y))+d(\rho(g \cdot y), g \cdot f(y)) \\
& =d(f(g \cdot y), \rho(g \cdot y))+d(g \cdot \rho(y), g \cdot f(y)) \\
& <\epsilon
\end{aligned}
$$

so that $f$ is equivariant, as required.

Example 6.3 Consider the scaling and translation action of $\mathbb{R}^{2}$ given on a equivariant family of Lipschitz continuous curves $(x, y(x))$ in the plane by $g \cdot(x, y(x))=$ $(x, \exp (\lambda) y+k)$. A smooth frame is given by $g \cdot y=0, g \cdot y_{x}=1$, or $\left.(\exp (\lambda), k)\right|_{\rho}=$ $\left(1 / y_{x},-y / y_{x}\right)$; the domain of this frame has $y_{x}>0$. Suppose now we wish to discretise this frame in a way that is compatible with the smooth frame and with forward difference, that is $y_{n+1}=y_{n}+\alpha y_{x}$. Then the discrete frame $\rho_{n}$ would be obtained by the normalisation equations, $g \cdot y_{n}=0, g \cdot y_{n+1}=\alpha$, so that

$$
\rho_{n}=\left(\frac{\alpha}{y_{n+1}-y_{n}}, \frac{-\alpha y_{n}}{y_{n+1}-y_{n}}\right) \text {. }
$$

This family of frames is straightforwardly seen to be equicontinuous, to have the smooth frame as its continuum limit, and to have the smooth Maurer-Cartan invariants as the limit of the discrete ones, provided the parameter $\alpha$ scales as the mesh size $x_{n+1}-x_{n}$.

\section{References}

1. N. Bila, E.L. Mansfield and P.A. Clarkson, Symmetry group analysis of the shallow water and semigeostrophic systems, Quarterly journal of Mechanics and Applied Mathematics, 59 (2006), 95-123. 
2. G. Bluman and S. Kumei, Symmetries and Differential Equations, volume 154 of Applied Mathematical Sciences. Springer Verlag, New York, 1989.

3. A.I. Bobenko and Y.B. Suris. Discrete differential geometry. Consistency as integrability, Graduate Studies in Mathematics, Vol. 98. AMS, 2008.

4. A. Bobenko and U. Pinkall, Discrete surfaces with constant negative Gaussian curvature and the Hirota equation, J. Diff. Geometry 43 (1996), 527-611.

5. M. Boutin, On orbit dimensions under a simultaneous Lie group action on $n$ copies of a manifold, J. Lie Theory 12 (2002), 191-203.

6. A. Calini, T. Ivey and G. Marí Beffa, An integrable flow for starlike curves in centroaffine space, Symmetry, Integrability and Geometry: Methods and Applications 9 (2013), 022.

7. É. Cartan, Oeuvres complètes, Gauthier-Villiars, 1952-55.

8. S.S. Chern and K. Tenenblat, Pseudo-spherical surfaces and evolution equations, Stud. Appl. Math. 74 (1986), 55-83.

9. É. Cotton, Généralization de la théorie de trièdre mobile', Bull. Soc. Math. France 33 (1905), 1-23.

10. C. de Boor, Polynomial interpolation in several variables, in: Studies in Computer Science: In Honour of Samuel D. Conte. J.R. Rice and R. DeMillo (eds.), Plenum Press, 1994 and Springer Science+Business Media New York, 1994, 87-110.

11. C. de Boor and A. Ron, On Multivariate Polynomial Interpolation, Constr. Approx. 6 (1990), $287-302$.

12. C. de Boor and A. Ron, The least solution for the polynomial interpolation problem, Mathematische Zeitschrift 210 (1992), 347-378.

13. C. de Boor and A. Ron, Computational aspects of polynomial interpolation in several variables, Mathematics of Computation 58 (1992), 705-727.

14. G. Darboux, Leçons sur la theorie générale des surfaces et des applications géometrique du calcul infinitésimal, Gauthier-Villars, 1887.

15. J. Dugunji, Topology, Allyn and Bacon Inc., Boston, 1966.

16. M. Fels and P.J. Olver, Moving Coframes I, Acta Appl. Math. 51 (1998), 161-213.

17. M. Fels and P.J. Olver, Moving coframes. II. Regularization and theoretical foundations Acta Appl. Math. (1999), pp 127-208.

18. M. Gasca and J.I. Maeztu, On Lagrange and Hermite interpolation in $\mathbb{R}^{k}$, Numerische Mathematik 39 (1982), 1-14.

19. M. L. Green, The moving frame, differential invariants and rigidity theorems for curves in homogeneous spaces, Duke Math. J. 45 (1978), 735-779.

20. P. Griffiths, On Cartan's methods of Lie groups and moving frames as applied to uniqueness and existence questions in differential geometry, Duke Math. J. 41 (1974), 775-814.

21. T.M.N. Gonçalves and E.L. Mansfield, On Moving frames and Noether's Conservation Laws, Studies in Applied Mathematics 128 (2011), 1-29.

22. T.M.N. Gonçalves and E.L. Mansfield, Moving Frames and Conservation Laws for Euclidean Invariant Lagrangians, Studies in Applied Mathematics 130 (2013), 134-166.

23. M.S. Hickman, W.A. Hereman, Computation of densities and fluxes of nonlinear differential-difference equations, Proceedings of the Royal Society A 457 (2003), 2705-2729.

24. R. Hasimoto, A soliton on a vortex filament, J. Fluid Mech. 51 (1972), 477-485.

25. K. Hikami and R. Inoue, Classical lattice W algebras and integrable systems, J. Phys. A: Math. Gen. 30 (1997), 6911-6924.

26. M.W. Hirsch, Differential Topology, Springer Verlag, New York, 1976.

27. E. Hubert, Differential Algebra for Derivations with Nontrivial Commutation Rules, J. of Pure and Applied Algebra 200 (1-2) (2005), 163-190.

28. E. Hubert, Differential invariants of a Lie group action: syzygies on a generating set, J. Symbolic Computation 44(4) (2009), 382-416.

29. E. Hubert, Generation properties of Maurer-Cartan invariants, (2009) Preprint [hal:inria00194528/en].

30. E. Hubert and I.A. Kogan, Smooth and Algebraic Invariants of a Group Action. Local and Global Constructions, Foundations of Computational Mathematics 7 (4) (2007), 345-383.

31. E. Hubert and I. A. Kogan, Rational Invariants of a Group Action. Construction and Rewriting, Journal of Symbolic Computation 42 (2007), 203-217.

32. P.E. Hydon and E.L. Mansfield, A variational complex for difference equations, Foundations of Computational Mathematics 4 (2004), 187-217. 
33. P.E. Hydon, E.L. Mansfield, L. Peng, and A. Rojo, Discrete moving frames and finite difference variational problems, in preparation.

34. P. Kim and P.J. Olver, Geometric integration via multi-space, Regular and Chaotic Dynamics 9(3) (2004), 213-226.

35. P. Kim, Invariantization of Numerical Schemes Using Moving Frames, BIT Numerical Mathematics 47(3) (2007), 525.

36. P. Kim, Invariantization of the Crank-Nicolson Method for Burgers' Equation, Physica D: Nonlinear Phenomena 237(2) (2008), 243.

37. I.A. Kogan and P.J. Olver, Invariant Euler-Lagrange equations and the invariant variational bicomplex, Acta Appl. Math. 76 (2003), 137-193.

38. E.L. Mansfield, A practical guide to the invariant calculus, Cambridge Monographs on Applied and Computational Mathematics Volume 26, Cambridge University Press, 2010.

39. E.L. Mansfield and P.E. Hydon, Difference Forms, Foundations of Computational Mathematics 8 (2008), 427-467.

40. E.L. Mansfield and P. van der Kamp, Evolution of curvature invariants and lifting integrability, J. Geometry and Physics 56 (2006), 1294-1325.

41. E.L. Mansfield and P.E. Hydon, Towards approximations of difference equations that preserve integrals, Proc. 2001 International Symposium on Symbolic and Algebraic Manipulation (ISSAC 2001) ed., B. Mourrain, ACM, New York, (2001), 217-222.

42. E.L. Mansfield, G. Marí Beffa, and J.P. Wang, Discrete moving frames and integrable systems, Foundations of Computational Mathematics 13, Issue 4 (2013), 545-582.

43. G. Marí Beffa, Geometric Hamiltonian structures on flat semisimple homogeneous manifolds, the Asian Journal of Mathematics 12(1) (2008), 1-33.

44. G. Marí Beffa, Poisson geometry of differential invariants of curves in some nonsemisimple homogenous spaces, Proc. Amer. Math. Soc. 134 (2006), 779-791.

45. G. Marí Beffa, On bi-Hamiltonian flows and their realizations as curves in real semisimple homogeneous manifolds, Pacific Journal of Mathematics 247 (2010), 163-188.

46. G. Marí Beffa, Hamiltonian evolutions of twisted polygons in parabolic manifolds: the Lagrangian Grassmannian, Pacific Journal of Mathematics 270 (2014), 287-317.

47. G. Marí Beffa, and J.P. Wang, Hamiltonian evolutions of twisted gons in $R P^{n}$, Nonlinearity 26 (2013), 2515-2551.

48. J. Munkres, Topology, Prentice-Hall, New Jersey, Second edition, 2000.

49. E. Noether, Invariant variation problems, Transport Theory Statist. Phys., 1(3):186-207, 1971. Translated from the German (Nachr. Akad. Wiss. Göttingen Math.-Phys. K1. II 1918, 235-257).

50. P.J. Olver, Moving frames - in geometry, algebra, computer vision, and numerical analysis, Foundations of Computational Mathematics, R. DeVore, A. Iserles and E. Suli, eds., London Math. Soc. Lecture Note Series, vol. 284, Cambridge University Press, Cambridge, 2, 2001, 67-297.

51. P.J. Olver, Geometric Foundations of Numerical Algorithms and Symmetry, Appl. Alg. Engin. Comp. Commun. 11 (2001), 417-436.

52. P.J. Olver, Joint invariant signatures, Foundations of Computational Mathematics 1 (2001), 3-67.

53. P.J. Olver, Applications of Lie groups to differential equations, Graduate Texts in Mathematics 107, Springer Verlag, New York, Second edition, 1993.

54. P.J. Olver and J. Pohjanpelto, Moving frames for Lie pseudo-groups, Canadian J. Math. 60 (2008), 1336-1386.

55. U. Pinkall, Hamiltonian flows on the space of star-shaped curves, Result. Math. 27 (1995), 328-332.

56. R. Rebelo and F. Valiquette, Symmetry preserving numerical schemes for partial differential equations and their numerical tests, J. Dif. Eq. and App., 19 (2013), 738-757.

57. E.G. Reyes, Pseudo-spherical surfaces and integrability of evolution equations, J. Diff. Eq. 147 (1998), 195-230.

58. C.L. Terng and K. Uhlenbeck, Schrödinger flows on Grassmannians, Integrable systems, geometry and topology, AMS/IP Stud. Adv.Math, AMS, Providence (2006), 235-256.

59. L.W. Tu, Une courte démonstration de la formule de Campbell-Hausdorff, Journal of Lie Theory 14 (2004), 501-508.

60. H. Wendland, Scattered Data Approximation, Cambridge Monographs on Applied and Computational Mathematics Volume 17, Cambridge University Press, 2005. 